EUROPEAN CENTRAL BANK

WORKING PAPER SERIES

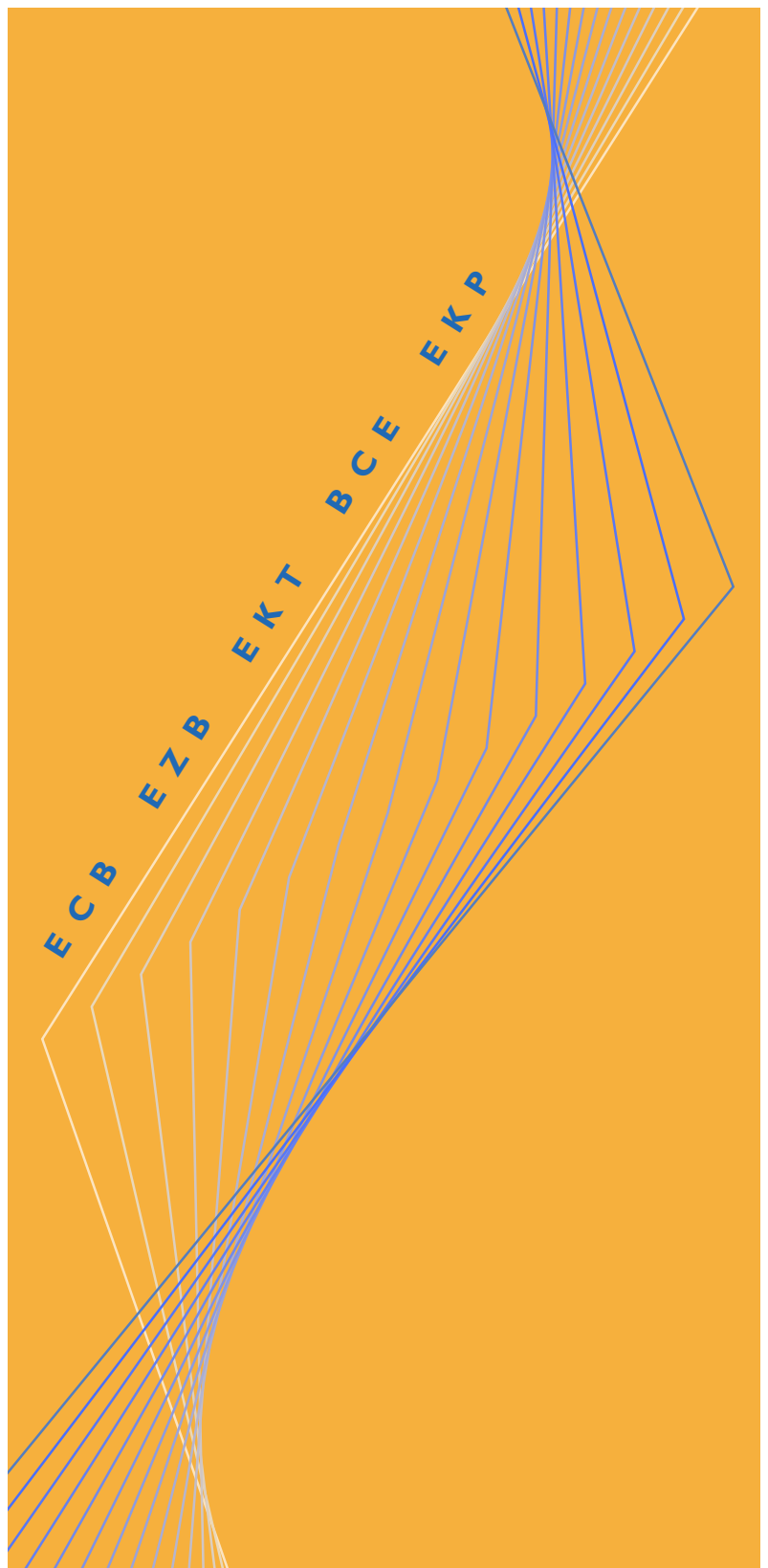

WORKING PAPER NO. I I 9

MONETARY POLICY AND

THE STOCK MARKET IN THE EURO AREA

\title{
BY NUNO CASSOLA AND
}

CLAUDIO MORANA

January 2002 


\title{
WORKING PAPER SERIES
}

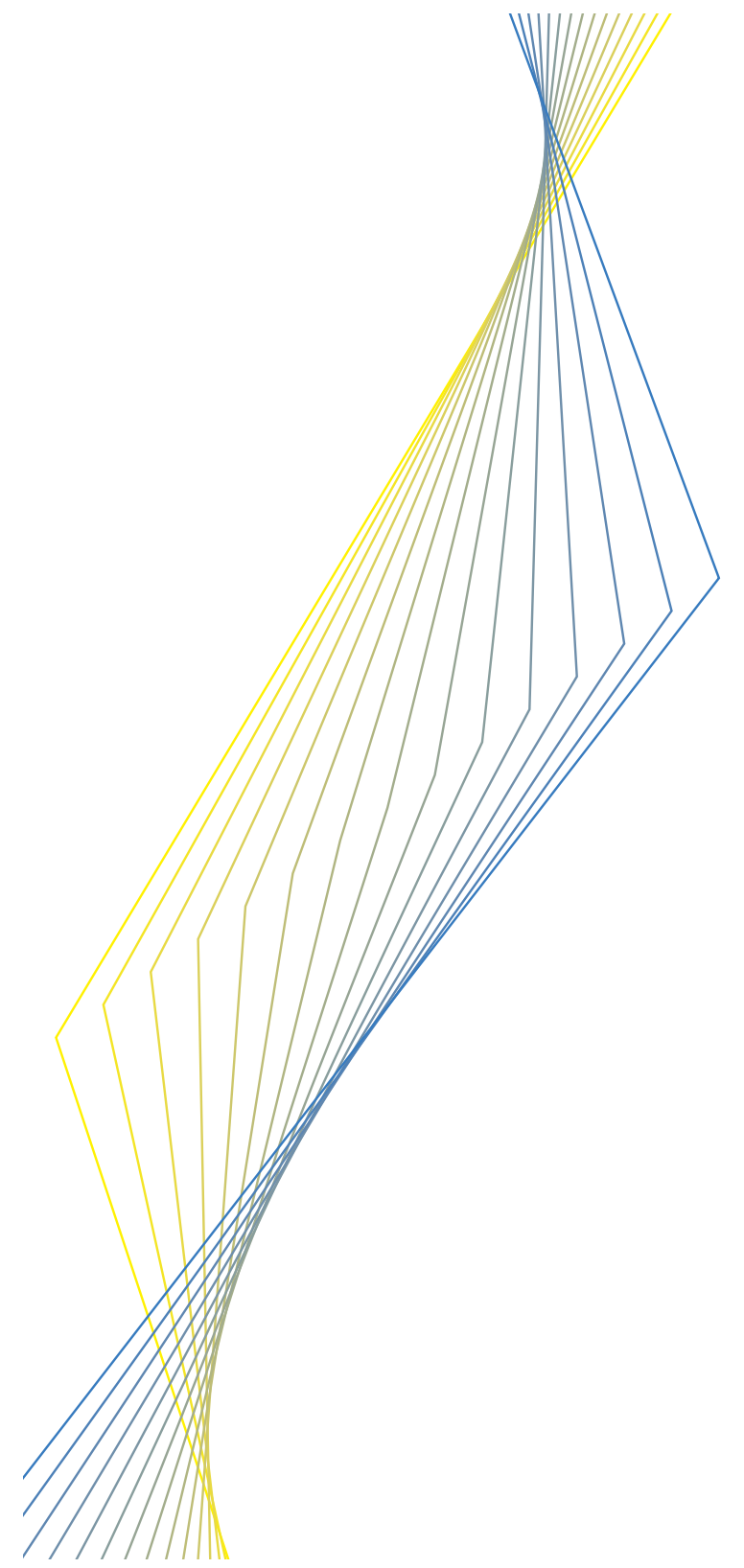

\author{
WORKING PAPER NO. I I9 \\ MONETARY POLICY AND \\ THE STOCK MARKET IN THE \\ EURO AREA
BY NUNO CASSOLA* AND CLAUDIO MORANA**

January 2002

The authors are grateful to an anonymous referee, A. Beltratti, F.C. Bagliano, R. Mosconi, and to participants in ECB internal seminars for comments and suggestions and to Manfred Kremer who saved us from some mistakes. Remaining errors are, of course, our own. 
(C) European Central Bank, 2002

\begin{tabular}{|c|c|}
\hline \multirow[t]{2}{*}{ Address } & Kaiserstrasse 29 \\
\hline & D-603 I I Frankfurt am Main \\
\hline & Germany \\
\hline \multirow[t]{3}{*}{ Postal address } & Postfach 160319 \\
\hline & D-60066 Frankfurt am Main \\
\hline & Germany \\
\hline Telephone & +496913440 \\
\hline Internet & http://www.ecb.int \\
\hline Fax & +496913446000 \\
\hline Telex & 4 I I I44 ecb d \\
\hline
\end{tabular}

Reproduction for educational and non-commercial purposes is permitted provided that the source is acknowledged.

The views expressed in this paper are those of the authors and do not necessarily reflect those of the European Central Bank.

ISSN $156 \mid-0810$ 


\section{Contents}

$\begin{array}{lr}\text { Abstract } & 4\end{array}$

$\begin{array}{lr}\text { Non-technical summary } & 5\end{array}$

$\begin{array}{lr}\text { I Introduction } & 7\end{array}$

2 Theoretical framework $\quad 8$

2.I The theoretical model 9

2.2 Monetary policy 13

$\begin{array}{lll}3 & \text { Econometric methodology } & \text { I4 }\end{array}$

4 Empirical results $\quad 19$

4.I Cointegration analysis $\quad 20$

$\begin{array}{ll}4.2 \text { Short run dynamics } & 22\end{array}$

4.3 Common trends analysis $\quad 23$

4.4 A closer look at cyclical dynamics $\quad 30$

4.5 A closer look at the stock market $\quad 32$

5 Implications for monetary policy $\quad 35$

5.I Price stability and financial stability: consistency suggested by empirical evidence 35

5.2 Price stability and financial stability: consistency suggested by a simulation exercise 36

$\begin{array}{llr}6 & \text { Conclusions } & 39\end{array}$

$\begin{array}{lr}\text { References } & 39\end{array}$

7 Appendix: the theoretical model $\quad 45$

7.I The steady-state of the model $\quad 45$

$\begin{array}{ll}7.2 \text { Short-run predictions of the model } & 50\end{array}$

$\begin{array}{ll}\text { Tables } & 53\end{array}$

$\begin{array}{ll}\text { Figures } & 62\end{array}$

$\begin{array}{ll}\text { European Central Bank Working Paper Series } & 78\end{array}$ 


\begin{abstract}
In this paper we study the role of the stock market in the transmission mechanism in the euro area and evaluate whether price stability and financial stability are mutually consistent and complementary objectives. Four major conclusions can be drawn from our work. First, stock prices and, more generally, relative asset prices seem to play an important role in the transmission mechanism in the euro area. Second, we do not find any significant, direct impact of stock prices on inflation. These two findings taken together support the view that stock market prices may be important for monetary policy, independently of their direct impact on inflation. Third, permanent productivity shocks are the driving force of the stock market in the long-run and contribute significantly to its cyclical behaviour. Nevertheless, the bulk of cyclical dynamics in the stock market is explained by transitory shocks. Fourth, a monetary policy focused on maintaining price stability in the long-run can contribute also to stock market stability.
\end{abstract}

J.E.L. classification: C32, O11.

Key words: monetary policy transmission mechanism, price stability, financial stability. 


\section{Non-technical summary}

We analyse within the framework of a small macro econometric model for the euro area, the interaction between nominal variables (nominal interest rates and inflation) and real variables (output, real M3 balances and real stock market prices). The purpose is to improve our understanding of the role of the stock market in the transmission mechanism of monetary policy in the euro area, and to evaluate its leading indicator properties for inflation and output growth. Furthermore, we assess empirically whether a medium- to long-term price stability objective is consistent with a smooth development of the stock market in the euro area. The dynamic behaviour of the economy is explained by six structural shocks: persistent supplyside shock, persistent shock to the inflation objective of the monetary authority, temporary aggregate demand shock, temporary liquidity preference shock, temporary term structure shock, and temporary shock to the short-term real interest rate.

We follow the structural vector error correction modelling (SVECM) approach where the economic variables are represented as the sum of a trend/permanent component and a cyclical component. The permanent shocks affect both the trend and the cyclical components whereas the temporary shocks affect only the cyclical component. We interpret monetary policy as involving two dimensions. The first dimension, medium- to long-term, is the setting of the inflation objective reflected in our model in the excess nominal M3 growth process (trend inflation which is equal to nominal M3 growth above what is needed to finance output trend growth). The second, short-term, is maintaining inflation on track by changing short-term interest rates, taking into account all shocks hitting the economy. A third component is added capturing non-systematic determinants of monetary policy. A crucial point in the identification of the latter component is that short-term real interest rates must (temporarily) increase in response to a (temporary) monetary policy tightening.

The dynamic behaviour of the economy is summarised, in the paper, by forecast error variance decomposition exercises and impulse-response analysis. In the short- to mediumterm, aggregate demand shocks are the main source of output variability whilst supplyside/productivity shocks are the main source of output variability in the long-term. Liquidity preference shocks are the most important determinant of real stock market price index volatility in the short-term, whilst productivity shocks are the main source of stock market variability in the medium- to long-term. In the short-term the supply-side/productivity shock is the main source of variability of the bond yield, whilst the persistent shock to the inflation objective of the monetary authority is the main source of variability in the medium- to longterm. The positive productivity shock has a temporary deflationary impact on the economy. The permanent shock to the inflation objective of the monetary authority has a persistent impact on the bond yield, which increases due to the Fisher effect. 
Historical decomposition of output and stock market cycles highlights its likely underlying determinants (structural shocks). The contribution of the aggregate demand shock is more important than supply-shocks in 1989-1991, around the timing of German unification. After 1997 the supply-side shock played a greater role in explaining the output cycle. In short, the most important determinants of cyclical dynamics in the euro area were demand shocks in the late 1980s early 1990s and supply-side shocks at the turn of the century. The stock market cycle can be explained to a large extent by productivity shocks. However, productivity shocks do not fully account for the behaviour of the stock market over the cycle. In 19881994 aggregate demand shocks played an important role in explaining the stock market cycle. Additionally, liquidity preference shocks played an important role in 1985-1988 which suggest that "pure" speculative behaviour may have been important in the period around the stock market crash of 1987. In 1999, monetary policy shocks (real short-term interest rate shocks) seem to have had an important role in explaining the stock market cycle.

To investigate the consistency between price stability and the smooth development of the stock market we use the estimated SVAR to generate artificial euro area data under a price stability environment and compare it with the actual data. To carry out the policy simulations we derive a monetary policy rule for controlling trend inflation in the SVECM. The rule is very simple: at each period the interest rate is changed proportional to deviation of current trend inflation from desired trend inflation. In our model it turns out that this rule is equivalent to calculating the change in the rate of growth of nominal M3 needed to bring trend inflation in line with the aim. The policy is implemented in the model as a temporary shock to the short-term real interest rate sufficiently strong to correct inflation as desired.

We start with an unconditional control exercise applied to the 1987-2000 period. As expected, the historical interest rate series is on average lower than the levels that would have been consistent with the aim of achieving inflation rates below $2 \%$. However, according to our model, since mid-1996 the short-term interest rate has been set at a level that broadly aims at increases in the GDP deflator between $1.5 \%$ and $2 \%$ in the euro area. Next we simulate euro area data assuming that the control rule is actually implemented starting from 1987. The euro area economy is exposed to historical shocks (except the short-term interest rate) to obtain the time path of the variables under the new regime. A successful inflation control policy would have smoothed, without avoiding, cyclical fluctuations. In addition, the initial output costs of disinflation, relative to the realised output cycle, tend to zero on average over the sample considered, since the initial negative impact of monetary policy tightening on the output gap in the late 1980s is followed by a positive impact in the 1990s. 


\section{Introduction}

Recent world wide stock market volatility has revived interest in understanding the possible role of central banks in preventing or reducing the disruptive effects of financial shocks on the economy. In fact, beyond the need to better understand the role of stock prices in the transmission mechanism, monetary policy should take stock prices into account as large swings in stock prices, either related or unrelated to fundamentals, may have a destabilising impact on the economy. This idea and its implications for monetary policy has been formalised and discussed in a recent paper by Bernanke and Gertler (1999), who recommend that price stability should be the overriding long-run goal of monetary policy, in order to avoid stock market volatility. This conclusion is challenged by Cecchetti et al. (2000) who recommend that central banks should react directly to equity price movements. ${ }^{1}$ In fact, these authors suggest that a central bank concerned with price stability should be preemptive and take explicitly into account asset prices, as well as other economic indicators, when making monetary policy decisions. This strategy should improve macroeconomic performance (reduce the variability of output and inflation), avoiding large asset price misalignments, boom and bust investment cycles, inflation and employment instability.

In this paper we make a contribution to the current debate on these policy issues. Furthermore, as noted by Sellin (2001) empirical analyses of the relation between stock market prices and monetary policy are needed, particularly with carefully identified monetary policy and performed on non-US data. Thus, we analyse within the framework of a small macroeconometric model for the euro area, the interactions between nominal variables (nominal interest rates and inflation) and real variables (output, real M3 balances and real stock market prices). The purpose is twofold. First, to improve our understanding of the monetary transmission mechanism in the euro area and, in particular, the role of the stock market. Second, to assess whether medium-term price stability is consistent with a smooth development of the stock market in the euro area.

Four major conclusions can be drawn from our work. First, stock prices and, more generally, relative asset prices seem to play an important role in the transmission mechanism in the euro area. In fact, output dynamics depends on the slope of the yield curve and on a measure of deviation of stock market prices from

\footnotetext{
${ }^{1}$ However, according to Cecchetti et al. (2000), central banks should not include asset prices in the objective of monetary policy. Goodhart (1999) considers asset prices directly in a broader measure of price stability along the lines of Alchian and Klein (1973).
} 
equilibrium. Second, we do not find any significant, direct impact of stock prices on inflation. These two findings taken together support the view that stock market prices may be important for monetary policy, independently of their direct impact on inflation. Third, permanent productivity shocks are the driving force of the stock market in the long-run and contribute significantly to its cyclical behaviour. Nevertheless, the bulk of cyclical dynamics in the stock market is explained by transitory shocks. Fourth, a monetary policy focused on maintaining price stability in the long-run can contribute also to stock market stability.

The remainder of the paper is organised as follows. In sections 2 and 3 we introduce the theoretical framework and the econometric methodology, respectively. In section 4 we present the data and the econometric results of cointegration and common trends analysis. Section 5 contains the main conclusions.

\section{Theoretical framework}

A booming stock market may have a positive impact on aggregate demand through four main channels. The first channel has been suggested by Tobin (1969), and operates through the impact that rising share prices have on the cost of capital boosting firm's investment. In fact, when share prices are high, the value of the firm relative to the replacement cost of its stock of capital (Tobin's q) is also high. Firms can therefore easily finance investments with relative small issues of new shares. This leads to increased investment spending, aggregate demand and output. The second channel has been suggested by Modigliani (1971) and operates through the impact of wealth on consumption. A permanent increase in asset prices implies an increase in wealth and therefore in permanent income. In an intertemporal framework where consumers smooth consumption over time, wealth effects lead to higher current and future consumption, stimulating aggregate demand and output. The third channel suggests that asset prices can also affect consumption through a cash flow mechanism. As asset prices increase, consumers will regard the possibility of finding themselves in financial distress less likely. They will therefore hold less liquid assets increasing their expenditure on durables and housing. Finally, the fourth channel operates through the impact that improved firm's balance sheets have on investment. Because of asymmetric information in credit markets, ability of firms to borrow depends on the value of the collateral they can offer. As the value of the collateral increases, the ability to borrow and invest increases. A self-reinforcing process can then take place when part of the available credit is employed also to purchase assets, leading to further 
price increases. This latter process, known as financial accelerator, has recently been discussed in a number of theoretical papers, as Bernanke and Gertler (1989), Kyotaki and Moore (1997) and Bernanke et al. (1998).

\subsection{The theoretical model}

Our modelling strategy is based on the idea that the rich and complex interaction between the real and the nominal sides of the economy implied by the various channels of the transmission mechanism can be captured, empirically, within a structural vector error correction model (SVECM). In designing such a model special attention has to be paid to long-run relations between economic variables. These relations, based on economic theory, imply a number of (testable) overidentifying restrictions on an otherwise unrestricted vector autoregressive model (VAR).

The economy is represented by six endogenous variables measured in natural logarithms: real GDP $\left(y_{t}\right)$, inflation defined as, $p_{t}-p_{t-1}=\pi_{t}$ where $p_{t}$ is the GDP deflator, real M3 balances $\left(m_{t}-p_{t}=r m_{t}\right)$, short-term interest rate $\left(i_{t}\right)$, bond yield $\left(l_{t}\right)$, and real stock market price index $\left(f_{t}\right)$ which is the log of the stock market price index deflated by the GDP deflator. Below we provide the basic structure of the model. A detailed derivation of the equations can be found in the appendix.

\subsubsection{The steady-state of the model}

In this section, the long-run restrictions used as building blocks in setting up the SVECM are explained.

Fundamental stochastic processes Two underlying stochastic processes drive the endogenous variables in the long-run, namely a technology variable $\left(\theta_{t}\right)$ and a nominal variable $\left(\beta_{t}\right)$ related to the long-run inflation objective of the monetary authorities. These variables evolve over time according to the laws of motion

$$
\begin{gathered}
\theta_{t}=k_{\theta}+\theta_{t-1}+v_{\theta, t} \\
\beta_{t}=\beta_{t-1}+v_{\beta, t},
\end{gathered}
$$

where $v_{\theta, t}$ and $v_{\beta, t}$ are uncorrelated white noise processes and $k_{\theta}>0$ is a parameter. Thus, $\theta_{t}$ and $\beta_{t}$ are $I(1)$ processes. 
Aggregate supply The supply side of the economy (AS) is described by

$$
\pi_{t}=\pi_{t}^{*}+\alpha_{1}\left(y_{t}-y_{t}^{*}\right),
$$

where expected inflation $\left(\pi_{t}^{*}\right)$ and potential output $\left(y_{t}^{*}\right)$, defined as $\lim _{h \rightarrow \infty} E_{t}\left(\pi_{t+h}\right)=$ $\pi_{t}^{*}$ and $\lim _{h \rightarrow \infty} E_{t}\left(y_{t+h}\right)=y_{t}^{*}$, evolve over time according to

$$
\begin{gathered}
y_{t}^{*}=\gamma_{1} \theta_{t} \\
\pi_{t}^{*}=\gamma_{2} \beta_{t}+\gamma_{3} \theta_{t},
\end{gathered}
$$

and where $\alpha_{1} \geq 0, \gamma_{1} \geq 0, \gamma_{2} \geq 0, \gamma_{3} \geq 0$ are parameters.

Apart from the supply equation our model builds on portfolio balance and arbitrage relations.

Money demand Based on portfolio theory, money demand can be specified as a function of a scale factor and a vector of relative rates of return

$$
r m_{t}=\phi_{1} y_{t}-\phi / \mathbf{s}_{t}
$$

where $\mathbf{s}_{t}$ is a vector of spreads of yields on alternative assets with the (own) rate of return on real balances and $\phi$ is a vector of parameters. As the model includes four assets (M3, short-term bills, long-term bonds and stocks), we assume that there is a stationary combination of three yield spreads $^{2}$ such that

$$
\phi / \mathbf{s}_{t}=\varepsilon_{m, t} \sim I(0),
$$

where

$$
\varepsilon_{m, t}=\rho_{1} \varepsilon_{m, t-1}+v_{s, t} \quad \rho_{1}<1 .
$$

and $v_{s, t}$ is white noise.

Term structure One arbitrage relationship links the yields on short-term bills and long-term bonds

$$
l_{t}=i_{t}+\phi_{l}+\varepsilon_{l, t},
$$

where $\varepsilon_{l, t}$ follows

$$
\varepsilon_{l, t}=\rho_{2} \varepsilon_{l, t-1}+v_{l, t} \quad \rho_{2}<1
$$

and $v_{l, t}$ is white noise.

\footnotetext{
${ }^{2}$ The validity of the assumption on the existence of a stationary combination of three yield spreads is tested against the data as explained in section 4.1 .
} 
Fisher parity Another arbitrage relationship links the rate of return on capital with the short-term interest rate

$$
i_{t}=\phi_{f p}+\phi_{3} \pi_{t}+\varepsilon_{i, t}
$$

where $\phi_{f p}$ is the sum of the real short-term interest rate and the inflation risk premium, and $\varepsilon_{i, t}$ is a stationary disturbance

$$
\varepsilon_{i, t}=\rho_{3} \varepsilon_{i, t-1}+v_{i, t} \quad \rho_{3}<1 .
$$

Stock market The third arbitrage relation, based on the present-value model, links the stock market and output

$$
f_{t}=\phi_{f}+\phi_{4} y_{t}+\varepsilon_{f, t}
$$

where $f_{t}$ is the log real stock market index and $\varepsilon_{f, t}$ is a stationary disturbance

$$
\varepsilon_{f, t}=\rho_{5} \varepsilon_{f, t-1}+v_{f, t} \quad \rho_{5}<1,
$$

and $v_{f, t}$ is white noise.

Steady-state of the model To find the steady-state of the model, the initial values of the exogenous variables are set equal to zero. Given that $v_{j, t}$ $j=\theta, \beta, \pi^{*}, f, l, r p, m, i, d, s$ are i.i.d. with zero mean, constant variances and zero covariances, in the steady state $y_{t}=y_{t}^{*}, f_{t}=f_{t}^{*}, \pi_{t}=\pi_{t}^{*}, v_{i, t}=\varepsilon_{i, t}=0$. Thus, ignoring constants, the steady-state is as follows

$$
\left[\begin{array}{c}
y_{t} \\
f_{t} \\
r m_{t} \\
i_{t} \\
l_{t} \\
\pi_{t}
\end{array}\right]=\left[\begin{array}{cc}
\gamma_{1} & 0 \\
\phi_{4} \gamma_{1} & 0 \\
\phi_{1} \gamma_{1} & 0 \\
\phi_{3} \gamma_{3} & \phi_{3} \gamma_{2} \\
\phi_{3} \gamma_{3} & \phi_{3} \gamma_{2} \\
\gamma_{3} & \gamma_{2}
\end{array}\right]\left[\begin{array}{c}
\theta_{t} \\
\beta_{t}
\end{array}\right]
$$

The steady-state displays monetary neutrality as the nominal trend $\left(\beta_{t}\right)$ does not have an impact on real variables $\left(y_{t}, f_{t}, r m_{t}\right)$ in the long-run.

\subsubsection{Short-run predictions of the model}

To investigate the short-run behaviour of the endogenous variables it is necessary to specify an additional equation for the determination of output in the short-run. 
IS equation We assume that the goods market equilibrium, in deviations from the steady state, is described by the following equation

$$
\begin{gathered}
y_{t}-y_{t}^{*}=-\alpha_{2}\left[\left(l_{t}-l_{t}^{*}\right)-\left(\pi_{t}-\pi_{t}^{*}\right)\right]+\alpha_{3}\left(f_{t}-f_{t}^{*}\right)+\varepsilon_{A D, t}, \\
\varepsilon_{A D, t}=\rho_{7} \varepsilon_{A D, t-1}+v_{A D, t} \quad \rho_{7}<1,
\end{gathered}
$$

which is an augmented IS equation where output is negatively related to the long-term real interest rate (investment component), positively related to the real stock market price index (Tobin's q / balance sheets component), and depends on a transitory shock, possibly related to fiscal policy.

Aggregate demand The joint equilibrium in financial and goods markets determines aggregate demand (AD). Assuming that the short-term interest rate is the policy variable, the short-term Fisher parity equation can be considerd as the rule for setting the short-term rate by the monetary authority. To derive the aggregate demand function we use the term structure relation, the short-term Fisher parity and the stock market equilibrium condition in equation (2.11). The aggregate demand in deviation from the steady state, is

$$
y_{t}-y_{t}^{*}=-\phi_{2}\left(\pi_{t}-\pi_{t}^{*}\right)-\phi_{5} \varepsilon_{i, t}+\phi_{7} \varepsilon_{A D, t}+\phi_{6} \varepsilon_{f, t}-\phi_{5} \varepsilon_{l, t},
$$

where $\phi_{7}=\frac{1}{1-\alpha_{3} \phi_{4}},-\phi_{2}=-\alpha_{2}\left(\phi_{3}-1\right) \phi_{7}, \phi_{5}=\alpha_{2} \phi_{7}, \phi_{6}=\alpha_{3} \phi_{7}$. If $\phi_{7}>0$ $\left(\alpha_{3} \phi_{4}<1\right)$, then $\phi_{5}$ and $\phi_{6}$ are positive. In addition, if $\phi_{3}>1$, then also $\phi_{2}$ is positive. We assume that these (stability) conditions are met. The economic interpretation of these assumptions is that the aggregate demand curve is negatively sloped in the $\left(y_{t}, \pi_{t}\right)$ space.

Short-term macroeconomic equilibrium The AD/AS model in deviations from the steady state can be written as follows

$$
\begin{gathered}
y_{t}-y_{t}^{*}=-\phi_{2}\left(\pi_{t}-\pi_{t}^{*}\right)-\phi_{5} \varepsilon_{i, t}+\phi_{7} \varepsilon_{A D, t}+\phi_{6} \varepsilon_{f, t}-\phi_{5} \varepsilon_{l, t}, \\
\pi_{t}-\pi_{t}^{*}=\alpha_{1}\left(y_{t}-y_{t}^{*}\right),
\end{gathered}
$$

and solved for the output and inflation gaps. The reduced form of the model is therefore

$$
\left[\begin{array}{c}
y_{t}-y_{t}^{*} \\
\pi_{t}-\pi_{t}^{*}
\end{array}\right]=\frac{1}{-\lambda}\left[\begin{array}{cccc}
\phi_{5} & -\phi_{7} & -\phi_{6} & \phi_{5} \\
\alpha_{1} \phi_{5} & -\alpha_{1} \phi_{7} & -\alpha_{1} \phi_{6} & \alpha_{1} \phi_{5}
\end{array}\right]\left[\begin{array}{c}
\varepsilon_{i, t} \\
\varepsilon_{A D, t} \\
\varepsilon_{f, t} \\
\varepsilon_{l, t}
\end{array}\right]
$$


where $-\lambda=-1-\alpha_{1} \phi_{2}$. Assuming $\phi_{3}>1$ and $\alpha_{3} \phi_{4}<1, \lambda$ is positive $(\lambda=$ $\left.1+\frac{\alpha_{1} \alpha_{2}\left(\phi_{3}-1\right)}{1-\alpha_{3} \phi_{4}}>0\right)$.

The model predicts that short- and long-term interest rate innovations have a negative impact on inflation and output, while demand and financial shocks have positive impacts on both variables. In addition, if $\alpha_{1}<1$ the impact on the inflation cycle will be smaller than the impact on the output cycle. Given the recursive structure of the economy, the reduced form for the other variables can be easily derived (see Appendix).

\subsection{Monetary policy}

There are two possible ways of thinking about monetary policy in the model. One is to consider that there is an underlying interest rate, directly (or closely) controlled by the central bank, $i_{t}^{c b}$, which is set taking into account all current available information ${ }^{3}$

$$
i_{t}^{c b}=i_{t-1}^{c b}+\Psi\left(i_{t}^{c b *}, \pi_{t}, \pi_{t}^{*}, y_{t}, f_{t}, r m_{t}, l_{t}, i_{t}\right)+\varepsilon_{c b, t},
$$

where $\left(i_{t}^{c b *}-\pi_{t}^{*}\right)$ denotes the equilibrium real central bank rate, and $\Psi($.$) describes$ the systematic behaviour of the monetary authority, after taking into account the desire to smooth the path of interest rates $\left(i_{t-1}^{c b}\right)$. An unexplained component in the behaviour of the central bank $\left(\varepsilon_{c b, t}\right)$ is also added. The inclusion of $\pi_{t}^{*}$ reflects the idea that the central bank pursues a medium- to long-run inflation objective, which can vary over time ${ }^{4}$. However, there must be a mechanism linking changes in $i_{t}^{c b}$ to changes in market interest rates along the yield curve

$$
\Delta i_{t}^{c b} \rightarrow \Delta i_{t} \rightarrow \Delta l_{t}
$$

which is not explicitly modelled given that $i_{t}^{c b}$ is not included in the data set.

An alternative way of thinking about monetary policy is to specify a growth rate for nominal M3. From the steady-state relations it is possible to derive an expression for the equilibrium rate of growth for nominal balances $\left(\Delta m_{n}^{*}\right)$. In fact

$$
\begin{aligned}
\Delta m_{n, t}^{*} & =\Delta r m_{t}^{*}+\pi_{t}^{*}=\phi_{1} \gamma_{1} \Delta \theta_{t}+\gamma_{2} \beta_{t}+\gamma_{3} \theta_{t} \\
& =\gamma_{2} \beta_{t}+\gamma_{3} \theta_{t}+\phi_{1} \gamma_{1} k_{\theta},
\end{aligned}
$$

\footnotetext{
${ }^{3}$ For simplicity we consider here that the information set of monetary authorities includes only current values of economic variables. In our application lagged values are also considered.

${ }^{4}$ The inclusion of a time-varying long-run inflation objective of the monetary authority is necessary to model the gradual disinflation process that is characteristic of the euro area in the 1980-1990 period.
} 
so that long-run inflation can be interpreted as the excess of nominal money growth relative to what is necessary to finance trend output growth

$$
\pi_{t}^{*}=\Delta m_{n, t}^{*}-\phi_{1} \gamma_{1} k_{\theta}=\gamma_{2} \beta_{t}+\gamma_{3} \theta_{t}
$$

Thus, if $\gamma_{3}=0$, the nominal trend can be interpreted as the excess nominal money growth process. In this case, given that $\Delta \beta_{t}=v_{\beta, t}$, and $\Delta^{2} m_{n, t}^{*}=\gamma_{2} \Delta \beta_{t}=\gamma_{2} v_{\beta, t}$, the term $v_{\beta, t}$ can be interpreted as reflecting changes in the long-run inflation objective.

The two ways of thinking about monetary policy are neither incompatible nor exclusive. In fact, we interpret monetary policy as involving two dimensions: one, medium- to long-term, is the setting of the inflation objective reflected in excess nominal growth of money $\left(\pi_{t}^{*}\right)$; another, short-term, is maintaining inflation on track by changing short-term interest rates taking into account all shocks hitting the economy $(\Psi()$.$) . The third component \left(\varepsilon_{c b, t}\right)$ captures non-systematic determinants of monetary policy.

\section{Econometric methodology}

In this section we sketch the empirical modelling strategy, which is based on SVAR analysis. Given a vector of $n I(1)$ cointegrated variables of interest $\mathbf{x}_{t}$, the vector error correction representation (VECM) can be written as:

$$
\Pi^{*}(L) \Delta \mathbf{x}_{t}=\boldsymbol{\nu}+\boldsymbol{\Pi} \mathbf{x}_{t-1}+\varepsilon_{t},
$$

where $\varepsilon_{t} \sim N I D(\mathbf{0}, \boldsymbol{\Sigma}), \boldsymbol{\Pi}(L)=\mathbf{I}_{n}-\sum_{i=1}^{p} \Pi_{i} L^{i}, \boldsymbol{\Pi}=-\boldsymbol{\Pi}(1), \boldsymbol{\Pi}^{*}(L)=\mathbf{I}_{n}-$ $\sum_{i=1}^{p-1} \Pi_{i}^{*} L^{i}$ and $\Pi_{i}^{*}=-\sum_{j=i+1}^{p} \boldsymbol{\Pi}_{j}(i=1, \ldots, p-1)$.

If there are $0<r<n$ cointegration relationships among the variables, $\Pi(1)$ is of reduced rank $r$ and can be expressed as the product of two $(n \times r)$ matrices: $\boldsymbol{\Pi}(1)=\boldsymbol{\alpha} \boldsymbol{\beta}^{\prime}$, where $\boldsymbol{\beta}$ contains the cointegrating vectors, such that $\boldsymbol{\beta}^{\prime} \mathbf{x}_{t}$ are stationary linear combinations of the $I(1)$ variables, and $\boldsymbol{\alpha}$ is the matrix of factor loadings.

The restricted vector autoregressive representation (RVAR) (Mellander, Vredin and Warne, 1992; Warne, 1993) can be written as

$$
\mathbf{B}(L) \mathbf{y}_{t}=\boldsymbol{\theta}+\boldsymbol{\eta}_{t}
$$

where $\mathbf{B}(L)=\mathbf{T}\left[\boldsymbol{\Pi}^{*}(L) \mathbf{T}^{-1} \mathbf{D}(L)+\boldsymbol{\alpha}^{*} L\right], \mathbf{y}_{t}=\mathbf{D}_{\perp}(L) \mathbf{T} \mathbf{x}_{t}, \boldsymbol{\theta}=\mathbf{T} \boldsymbol{\nu}, \boldsymbol{\eta}_{t}=\mathbf{T} \boldsymbol{\varepsilon}_{\mathbf{t}}$, $\mathbf{T}=\left[\begin{array}{ll}\boldsymbol{\beta}_{\perp}^{\prime} & \boldsymbol{\beta}\end{array}\right]^{\prime}, \boldsymbol{\alpha}^{*}=\left[\begin{array}{ll}\mathbf{0} & \boldsymbol{\alpha}\end{array}\right], \mathbf{D}(L)$ and $\mathbf{D}_{\perp}(L)$ are polynomial matrices 
defined by

$$
\mathbf{D}(L)=\left[\begin{array}{cc}
\mathbf{I}_{k} & \mathbf{0} \\
\mathbf{0} & (1-L) \mathbf{I}_{r}
\end{array}\right], \mathbf{D}_{\perp}(L)=\left[\begin{array}{cc}
(1-L) \mathbf{I}_{k} & \mathbf{0} \\
\mathbf{0} & \mathbf{I}_{r}
\end{array}\right] .
$$

Following Mellander, Vredin and Warne (1992) and Warne (1993), the RVAR can be inverted to obtain the common trends representation of Stock and Watson (1988), which, in structural form, can be written as

$$
\begin{aligned}
\mathbf{x}_{t} & =\mathbf{x}_{0}+\boldsymbol{\mu} t+\boldsymbol{\Gamma}(1) \sum_{j=0}^{t-1} \boldsymbol{\varphi}_{t-j}+\boldsymbol{\Gamma}^{*}(L) \boldsymbol{\varphi}_{t} \\
& =\mathbf{x}_{0}+\boldsymbol{\mu} t+\boldsymbol{\Gamma}_{g} \sum_{j=0}^{t-1} \boldsymbol{\psi}_{t-j}+\boldsymbol{\Gamma}^{*}(L) \boldsymbol{\varphi}_{t},
\end{aligned}
$$

where $\boldsymbol{\varphi}_{t} \equiv\left[\begin{array}{ll}\boldsymbol{\psi}_{t} & \boldsymbol{v}_{t}\end{array}\right]^{\prime} \sim$ I.I.D.(0, $\left.\mathbf{I}_{n}\right)$, with $\boldsymbol{\psi}_{t}$ and $\boldsymbol{v}_{t}$ subvectors of structural shocks of $k$ and $r$ elements respectively, $\varepsilon_{t}=\boldsymbol{\Gamma}_{0} \boldsymbol{\varphi}_{t}$, and $\boldsymbol{\Gamma}(1)=\sum_{j=0}^{\infty} \boldsymbol{\Gamma}_{j}$, $\boldsymbol{\Gamma}^{*}(L)=\sum_{j=0}^{\infty} \boldsymbol{\Gamma}_{j}^{*} L^{j}, \boldsymbol{\Gamma}_{j}^{*}=-\sum_{i=j+1}^{\infty} \boldsymbol{\Gamma}_{i}$, where $\boldsymbol{\Gamma}_{i}$ are matrices of parameters in the structural Wold vector moving average (VMA) representation. The existence of $r$ cointegrating vectors implies that the long-run matrix $\boldsymbol{\Gamma}(1)$ has rank $n-r \equiv k$ and $\boldsymbol{\beta}^{\prime} \boldsymbol{\Gamma}(1)=\mathbf{0}$.

In order to identify the elements of $\boldsymbol{\psi}_{t}$ as the permanent shocks and the elements of $\boldsymbol{v}_{t}$ as transitory disturbances, only the disturbances in $\boldsymbol{\psi}_{t}$ should be allowed to have long-run effects on (at least some of) the variables in $\mathbf{x}_{t}$. Hence, $\boldsymbol{\Gamma}(1)=\left[\begin{array}{ll}\boldsymbol{\Gamma}_{g} & 0\end{array}\right]$, being $\boldsymbol{\Gamma}_{g}$ a submatrix of dimension $n \times k$.

In the structural common trends representation only $k$ shocks (the permanent shocks $\boldsymbol{\psi}_{t}$ ) are cumulated in the trend component. The behaviour of the variables in $\mathbf{x}_{t}$ induced by permanent disturbances may then be computed as

$$
\mathbf{x}_{t}^{p}=\mathbf{x}_{0}+\boldsymbol{\mu} t+\boldsymbol{\Gamma}_{g} \sum_{j=0}^{t-1} \boldsymbol{\psi}_{t-j}
$$

while the cyclical components is

$$
\mathbf{x}_{t}^{c}=\Gamma^{*}(L) \varphi_{t} .
$$

This is the Beveridge-Nelson-Stock-Watson trend-cycle decomposition of $\mathbf{x}_{t}=\mathbf{x}_{t}^{p}+$ $\mathbf{x}_{t}^{c}$. Note that the cyclical component is determined by all the innovations in the system, both permanent and transitory. This implies that permanent innovations also induce transitory dynamics. 
Identification of the shocks To identify the common trends model it is necessary to find a matrix $\boldsymbol{\Gamma}_{0}$, such that it can be uniquely determined from the parameters of the VECM model in (3.1), where the variance covariance matrix of $\Gamma_{0}^{-1} \varepsilon_{t}=\varphi_{t}$ is diagonal with non zero entries, and the long-run impact matrix is $\Gamma(1)=\left[\begin{array}{ll}\boldsymbol{\Gamma}_{g} & 0\end{array}\right]$.

By rewriting the mapping from the reduced form disturbances to the structural disturbances as

$$
\Gamma_{0}^{-1} \varepsilon_{t}=\varphi_{t} \Leftrightarrow\left[\begin{array}{c}
\mathrm{G} \\
\mathbf{H}
\end{array}\right] \varepsilon_{t}=\left[\begin{array}{l}
\boldsymbol{\psi}_{t} \\
\boldsymbol{v}_{t}
\end{array}\right]
$$

it can be noticed that through the $(k \times n)$ matrix $\mathbf{G}$ the reduced form disturbances are mapped into permanent disturbances, and through the $(r \times n)$ matrix $\mathbf{H}$ the reduced form disturbances are mapped into transitory disturbances.

Following Warne (1993), the matrix $\mathbf{G}$ can be estimated as

$$
\mathbf{G}=\left(\boldsymbol{\Gamma}_{g}^{\prime} \boldsymbol{\Gamma}_{g}\right)^{-1} \boldsymbol{\Gamma}_{g}^{\prime} \mathbf{C}(1),
$$

where $\mathbf{C}(1)$ is the long-run impact matrix in the reduced form Wold VMA representation.

To estimate the $(n \times k)$ matrix $\boldsymbol{\Gamma}_{g}$, we need (at least) $n k$ restrictions on its elements. Cointegration implies

$$
\boldsymbol{\beta}^{\prime} \boldsymbol{\Gamma}_{g}=\mathbf{0},
$$

yielding $k r$ linear restrictions. Additional $k(k+1) / 2$ restrictions on the elements of $\boldsymbol{\Gamma}_{g}$ are provided by assuming $E\left(\boldsymbol{\psi}_{t} \boldsymbol{\psi}_{t}^{\prime}\right)=E\left(G \boldsymbol{\Sigma} \mathbf{G}^{\prime}\right)=\mathbf{I}_{k}$. That is, $k(k+1) / 2$ restrictions are given by

$$
\mathbf{C}(1) \Sigma \mathbf{C}(1)^{\prime}=\Gamma_{g} \Gamma_{g}^{\prime}
$$

since $\mathbf{C}(1)$ and $\boldsymbol{\Gamma}_{g}$ have reduced rank $k$. The remaining $k(k-1) / 2$ restrictions needed for (exact) identification of $\boldsymbol{\Gamma}_{g}$ have to be derived from economic theory.

To estimate the $(r \times n)$ matrix $\mathbf{H}$, we need (at least) $n r$ restrictions on its elements. It can be noticed that from the orthogonality condition $E\left[\boldsymbol{\psi}_{t} \boldsymbol{v}_{t}^{\prime}\right]=\mathbf{0}$ we have

$$
\mathbf{E}\left(\mathbf{G} \varepsilon_{t} \varepsilon_{t}^{\prime} \mathbf{H}^{\prime}\right)=\mathbf{G} \boldsymbol{\Sigma} \mathbf{H}^{\prime}=\mathbf{0},
$$

that is

$$
\left(\boldsymbol{\Gamma}_{g}^{\prime} \boldsymbol{\Gamma}_{g}\right)^{-1} \boldsymbol{\Gamma}_{g}^{\prime} \mathbf{C}(1) \Sigma \mathbf{H}^{\prime}=\mathbf{0}
$$

Hence, reminding that $\mathbf{C}(1) \boldsymbol{\alpha}=\mathbf{0}$, a possible solution for $\mathbf{H}$ takes the form

$$
\mathbf{H}=\mathrm{Q}^{-1} \boldsymbol{\zeta}^{\prime} \boldsymbol{\Sigma}^{-1},
$$


where $\boldsymbol{\zeta}=\boldsymbol{\alpha}(\mathbf{U} \boldsymbol{\alpha})^{-1}, \mathbf{U}$ is a matrix chosen in such a way that $\mathbf{U} \boldsymbol{\alpha}$ is non singular, and the $(r \times r)$ matrix $\mathbf{Q}$ is such that $E\left[\boldsymbol{v}_{t} \boldsymbol{v}_{t}^{\prime}\right]=\mathbf{I}_{r}$. In practice the matrix $\mathbf{Q}$ can be obtained from the Choleski decomposition of $\left(\boldsymbol{\zeta}^{\prime} \boldsymbol{\Sigma}^{-1} \boldsymbol{\zeta}\right)^{-1}$. The estimation of $\mathbf{H}$ requires the imposition of $r(r-1) / 2$ additional restrictions on the $(r \times r)$ matrix $\boldsymbol{\zeta}$, since the remaining $k r+r(r+1) / 2$ restrictions necessary for exact identification are provided by the orthogonality conditions $E\left[\boldsymbol{\psi}_{t} \boldsymbol{v}_{t}^{\prime}\right]=\mathbf{0}$ and $E\left[\boldsymbol{v}_{t} \boldsymbol{v}_{t}^{\prime}\right]=\mathbf{I}_{r}$.

By noting that $\boldsymbol{\Sigma}=\boldsymbol{\Gamma}_{0} \boldsymbol{\Gamma}_{0}^{\prime}$, we have that $\Gamma_{0}=\boldsymbol{\Sigma}\left(\boldsymbol{\Gamma}_{0}^{\prime}\right)^{-1}=\left[\begin{array}{ll}\boldsymbol{\Sigma} \mathbf{G}^{\prime} & \boldsymbol{\Sigma} \mathbf{H}^{\prime}\end{array}\right]$. Thus, the contemporaneous impact matrix can be written as

$$
\boldsymbol{\Gamma}_{0}=\left[\begin{array}{ll}
\boldsymbol{\Sigma} \mathbf{C}(1)^{\prime} \boldsymbol{\Gamma}_{g}\left(\boldsymbol{\Gamma}_{g}^{\prime} \boldsymbol{\Gamma}_{g}\right)^{-1} & \boldsymbol{\zeta}\left(\mathbf{Q}^{-1}\right)^{\prime}
\end{array}\right]
$$

When there exists a complete set of common cycles of order zero $(C S C C-0)$ (Engle and Vahid, 1993), the contemporaneous impact matrix can be written as

$$
\boldsymbol{\Gamma}_{0}=\left[\begin{array}{ll}
\boldsymbol{\Sigma} \tilde{\boldsymbol{\alpha}}\left(\boldsymbol{\Gamma}_{g}^{\prime} \tilde{\boldsymbol{\alpha}}\right)^{-1} & \tilde{\boldsymbol{\alpha}}_{\perp} \mathbf{S}^{\prime}
\end{array}\right]
$$

where $\mathbf{S}$ is a $(n-k) \times(n-r)$ normalization matrix such that $E\left[\boldsymbol{v}_{t} \boldsymbol{v}_{t}^{\prime}\right]=\mathbf{I}_{r}$, and the $n \times k$ matrix $\tilde{\boldsymbol{\alpha}}$ (the cofeature matrix) is such that $\tilde{\boldsymbol{\alpha}}^{\prime} \boldsymbol{\Gamma}_{i}^{*}=\mathbf{0} \forall i \geq 0, \tilde{\boldsymbol{\alpha}}^{\prime} \boldsymbol{\Gamma}(1)=\tilde{\boldsymbol{\alpha}}^{\prime}$. Therefore, as shown by Gallo and Kempf (1995), the $r(r-1) / 2$ additional a priori restrictions necessary to identify the transitory disturbances are not needed. This result implies that when common cycles characterize the data these restrictions must be taken into account when deriving the impulse response functions. A test for common cycles can be carried out following Engle and Vahid (1993), and should be performed before identifying the structural disturbances.

Impulse response functions Following Warne (1993), the impulse response functions for $\Delta \mathbf{x}_{t}$ and $\mathbf{y}_{t}$ can be computed, respectively, from

$$
\mathbf{R}(L)=\mathbf{C}(L) \boldsymbol{\Gamma}_{0}
$$

and

$$
\mathbf{G}(L)=\mathbf{F}(L) \boldsymbol{\Lambda}
$$

where $\mathbf{C}(L)=\mathbf{I}_{n}+\sum_{j=1}^{\infty} \mathbf{T}^{-1}\left(\mathbf{F}_{j}-\mathbf{D F} \mathbf{F}_{j-1}\right) \mathbf{T} L^{j}, \mathbf{D}=\mathbf{D}_{\perp}(1), \mathbf{B}(L)^{-1}=\mathbf{F}(L)=$ $\mathbf{I}_{n}+\sum_{j=1}^{\infty} \mathbf{F}_{j} L^{j}, \boldsymbol{\Lambda}=\mathbf{T} \boldsymbol{\Gamma}_{\mathbf{0}}$ 
Cyclical dynamics A key feature of the Beveridge-Nelson-Stock-Watson decomposition is that the cyclical component $\mathbf{x}_{t}^{c}$ is explained not only by transitory shocks, but also by permanent shocks. Proietti (1997) has proposed a methodology to disentangle in cyclical fluctuations the contribution of permanent shocks from the effect of transitory disturbances. The Proietti decomposition of the cyclical component can be restated as

$$
\mathbf{x}_{t}^{c}=\Gamma^{*}(L) \boldsymbol{\varphi}_{t}=\mathbf{F}_{1} \mathbf{c}_{1, t}+\mathbf{F}_{2} \mathbf{c}_{2, t}
$$

where

$$
\mathbf{F}_{1}=\left(\boldsymbol{\Phi}(1)+\boldsymbol{\alpha} \boldsymbol{\beta}^{\prime}\right)^{-1} \boldsymbol{\alpha}\left[\boldsymbol{\beta}^{\prime}\left(\boldsymbol{\Phi}(1)+\boldsymbol{\alpha} \boldsymbol{\beta}^{\prime}\right)^{-1} \boldsymbol{\alpha}\right]^{-1}
$$

is the $n \times r$ factor loading matrix of the $r \times 1$ mean adjusted error correction terms vector

$$
\mathbf{c}_{1, t}=\boldsymbol{\beta}^{\prime} \mathbf{x}_{t}-E\left[\boldsymbol{\beta}^{\prime} \mathbf{x}_{t}\right]
$$

and

$$
\mathbf{F}_{2}=-\left(\mathbf{I}_{n}-\mathbf{F}_{1} \boldsymbol{\beta}^{\prime}\right)\left(\boldsymbol{\Phi}(1)+\boldsymbol{\alpha} \boldsymbol{\beta}^{\prime}\right)^{-1}
$$

is the $n \times n$ factor loading matrix of the $n \times 1$ mean adjusted vector of changes

$$
c_{2, t}=\boldsymbol{\Phi}^{*}(L)\left(\Delta \mathbf{x}_{t}-E\left[\Delta \mathbf{x}_{t}\right]\right),
$$

where $\boldsymbol{\Phi}^{*}(L)=\sum_{i=0}^{p-2} \boldsymbol{\Phi}^{*} L^{i}, \boldsymbol{\Phi}_{j}^{*}=\sum_{i=j+1}^{p-1} \boldsymbol{\Phi}_{i}, \boldsymbol{\Phi}(L)=\mathbf{I}_{n}-\sum_{i=1}^{p-1} \boldsymbol{\Phi}_{i} L^{i}$, and $\boldsymbol{\Phi}_{j}=$ $-\mathbf{I}_{n}+\sum_{i=1}^{j} \boldsymbol{\Pi}_{j}(j=1, \ldots, p-1)$.

The proposed restatement of the Proietti decomposition allows determining the contribution of each primitive cycle generator component to the overall cycle. The vector $\mathbf{F}_{1} \mathbf{c}_{1, t}$ is the vector of dynamics towards the attractor set, while the vector $\mathbf{F}_{2} \mathbf{c}_{2, t}$ is the vector of dynamics along the attractor set. The dynamics towards the attractor set, by reflecting the working of the error correction mechanism, are out of equilibrium fluctuations. In particular, they are related to that portion of the cycle innovation that is orthogonal to the trend innovation. On the other hand, the dynamics along the attractor are fluctuations generated by that portion of the cycle innovation which is correlated with (and explained by) the trend innovation. In other words, the dynamics along the attractor may be thought of as the transitional dynamics which take place after a shock to the common trend hits the economy. Since along the attractor the cointegration relationships are satisfied, the dynamics along the attractor are equilibrium fluctuations. Note that the existence of a $C S C C-0$ implies that

$$
\left(\mathbf{I}_{n}-\mathbf{F}_{1} \boldsymbol{\beta}^{\prime}\right)\left(\boldsymbol{\Phi}(1)+\boldsymbol{\alpha} \boldsymbol{\beta}^{\prime}\right)^{-1} \boldsymbol{\Phi}_{i}=\mathbf{0} \quad i=1, \ldots, p-1,
$$


so that $\mathbf{F}_{2} \mathbf{c}_{2, t}=\mathbf{0}$ and the cyclical dynamics are fully explained by the error correction processes.

A similar decomposition of the cycles can be obtained by rewriting the vector of cyclical components as

$$
\mathbf{x}_{t}^{c}=\Gamma^{*}(L) \boldsymbol{\varphi}_{t}=\Gamma_{1}^{*}(L) \boldsymbol{\psi}_{t}+\boldsymbol{\Gamma}_{2}^{*}(L) \boldsymbol{v}_{t}
$$

The vector $\boldsymbol{\Gamma}_{1}^{*}(L) \boldsymbol{\psi}_{t}$ gives the contribution of permanent innovations to the overall cycle (dynamics along the attractor), while the vector $\boldsymbol{\Gamma}_{2}^{*}(L) \boldsymbol{v}_{t}$ measures the contribution of the transitory innovations to the overall cycle (dynamics towards the attractor). Note that the existence of a $C S C C-0$ implies that

$$
\Gamma_{1}^{*}(L) \boldsymbol{\psi}_{t}=\mathbf{0}
$$

in which case the cyclical dynamics are fully explained by the transitory innovations.

Differently from the Proietti decomposition, the latter approach allows assessing the contribution of each structural shock to cyclical fluctuations separately, and is, therefore, more informative from an economic point of view.

Further details on the estimation, identification and simulation of the common trends model are discussed in Warne (1993) and Gallo and Kempf (1995).

\section{Empirical results}

In this study quarterly data from 1980:Q1 through 2000:Q4 are used. ${ }^{5}$ As a measure of M3 quarterly averages of the month-end stocks of M3 are used (Source: ECB database, in millions of euro, seasonally adjusted by D-Statistics). Until 1997Q3 M3 data are based on stocks; from 1997Q4 on flow statistics. Nominal and real GDP until 1994Q4 is calculated based on ESA79 system of national accounts. From 1995Q1 the series is extended using ESA95 quarter-over-quarter growth rates. Nominal GDP is in millions of euro and has been seasonally adjusted and converted to euro via the irrevocable fixed conversion rates of 31 December 1998. The real and nominal GDP series are used to construct the GDP deflator. Shortterm rates are 3-month money market interest rates and long-term interest rates are 10 year government bond yields or close substitutes. From 1999 onwards the EURIBOR is used as 3-month money market rate. Interest rates are measured as

\footnotetext{
${ }^{5}$ Greece is not included in the data set due to lack of data at the time of writing.
} 
averages of the respective euro-11 interest rates using GDP weights at purchasing power exchange rates in 1995. Except interest rates, all data are in logs. The underlying national series are taken from the macroeconomic database provided by the BIS. The stock market index is taken from Datastream (TOTMKEM). We took the benchmark series expressed in USD and converted it into EUR using a synthetic USD/EUR exchange rate series. As a caveat, it should be noted that the resulting stock market series does not have the same aggregation scheme used to construct euro area output, inflation and monetary series.

\subsection{Cointegration analysis}

ADF unit root tests carried out on the levels of the variables suggest that all variables should be modelled as integrated $(I(1))$ processes. Two lags have been selected for the VAR in levels following a general to specific reduction approach. The parsimonious specification can be regarded as a valid approximation of the DGP on the basis of standard diagnostic tests carried out on the estimated residuals. As indicated in Table 1, the null of no cointegration can be rejected, at the $5 \%$ significance level, in favour of the alternative hypothesis of four cointegrating vectors. The identification structure selected is not rejected by the data.

According to the theoretical model, the identified long-run relationships can be interpreted as a long-run money demand equation, a term structure relation, a Fisher parity relation and a long run relationship between the real stock market index and real output. These results are broadly in line with previous work such as, Coenen and Vega (1999), Brand and Cassola (2000), Bagliano et al. (2001a,b), Golinelli and Pastorello (2000), who found evidence of stable long-run relations between the long-term interest rate and inflation, long- and short-term interest rates, and real money balances, real GDP and some measure of the opportunity cost of holding M3.

Irreducible cointegrating vectors The identified cointegrating vectors are irreducible (IC) according to the terminology proposed by Davidson (1998), that is, contain the smallest number of variables that ensures a stationary linear combination (e.g. two variables). The vectors can also be considered as structural, since they can be directly related to the theoretical long-run relations.

For the set of variables in the model, $\left\{y_{t}, r m_{t}, f_{t}, \pi_{t}, i_{t}, l_{t}\right\}$, we find four cointegration sets $\left\{y_{t}, r m_{t}\right\},\left\{y_{t}, f_{t}\right\},\left\{i_{t}, l_{t}\right\}$ and $\left\{\pi_{t}, i_{t}\right\}$. It follows that $\left\{r m_{t}, f_{t},\right\}$ and $\left\{l_{t}, \pi_{t}\right\}$ are also irreducible cointegrating vectors obtained by linear combinations 
of the appropriate vectors, where the common variables are eliminated (output and the short-term interest rate). The additional cointegrating vectors relate the real stock market index and real balances, and the long-term rate and the inflation rate, respectively. These relations are not structural in our theoretical framework and, therefore, should be considered as solved vectors. Furthermore, direct cointegration between output and real balances suggested by IC analysis can be interpreted as evidence in favour of the existence of a stationary combination of rates of return of the four assets (implicitly) included in our analysis.

This helps in understanding different findings regarding the specification of money demand in the euro area. For instance, Brand and Cassola (2000), include the long-term interest rate in the money demand function, which is a redundant variable in the money demand equation once the stock market variable is added to the system and according to IC analysis.

Separation An additional and important feature of the identified cointegration space is long-run separation as defined by Konishi and Granger (1992) and Granger and Haldrup (1997). In fact we can separate the cointegration space into two blocks, one involving only real variables $\left\{y_{t}, r m_{t}\right\}$ and $\left\{y_{t}, f_{t}\right\}$, and the other including only nominal variables, $\left\{i_{t}, l_{t}\right\}$ and $\left\{\pi_{t}, i_{t}\right\}$, each block of variables being driven by a different common stochastic trend. This is an important result that can be taken as evidence of long-run monetary neutrality. Separation is, however, not complete, since nominal and real variables interact in the short-run as will be shown in section 4.2 .

Weak exogeneity and implications for trend income velocity of M3 Weak exogeneity tests show that both excess real balances and stock market disequilibrium enter the dynamic equation for real money balances (see Table 1), yielding the following solved long-run money demand function

$$
r m_{t}=r m_{0}+1.4 y_{t}+0.06\left(f_{t}-4.7 y_{t}\right)
$$

or

$$
r m_{t}=r m_{0}+1.1 y_{t}+0.06 f_{t},
$$

which can be interpreted as a long-run relationship for income velocity of money

$$
y_{t}-r m_{t}=-r m_{0}-0.1 y_{t}-0.06 f_{t} \text {. }
$$


The negative relation between velocity and the real stock market price index can be justified, among other factors, by a wealth effect: a rise in real equity prices implies higher real wealth, which in turn leads to a higher wealth to income ratio. This tends to increase the demand for money relative to income (see Sellin (2001)). This empirical regularity, first noticed by Friedman (1988), is consistent with the results of Borio et al. (1994), who found that asset prices may account for the strong decline of income velocity of money, since the 1970s, in industrialised countries. Our estimate of the long-run impact of real stock prices on real money balances suggests a weaker response than what was found by Borio et al. (1994) for some major industrialised countries (Australia (0.50), Canada (0.14), Japan (0.19), Great Britain (0.19)) and a similar to the response for Norway (0.05) and Finland (0.10).

\subsection{Short run dynamics}

As shown in Table 1, the real stock market price index is the only variable that can be considered weakly exogenous. Real output reacts both to the interest rate spread and stock market disequilibrium, increasing when the short-term interest rate is below the long-term rate and when the real stock market index is above its long-run value determined by real GDP. These results support the view that asset prices are important in the transmission mechanism in the euro area.

Both inflation and the long-term interest rate correct relative to deviation from the Fisher parity relations, with inflation correcting at a higher speed.

The short-term interest rate corrects relative to the slope of the yield curve and excess real balances. The short-term interest rate falls when excess real balances increases. On the other hand, a steepening of the yield curve leads an increase in the short-term interest rate and an increase in inflation.

Cointegration between interest rates and inflation suggests that a single nominal permanent trend drives the three processes. Moreover, contrary to what was found by Vlaar and Schuberth (1998) and Bagliano et al. (2001b), we do not find evidence of short-run effects of excess real balances or stock market disequilibrium on inflation, at least when inflation is measured by changes in the (log) GDP deflator. The latter result is coherent with the general finding that stock market prices have little forecasting power for inflation (Goodhart and Hofman, 2000b; Filardo, 2000).

Finally, as shown in Table 1 and in Table 2, the rejection of complete separation between nominal and real variables is due not only to weak exogeneity failure, but 
also to the presence of bi-directional Granger causality between nominal and real variables. The crucial links seem to come through the real balances effect on the short-term interest rate and the term structure effect on output. Another interesting finding is the dependence of the stock market index on changes in the long-term interest rate (negative impact). The dynamic equations for the other variables are less informative.

Common cycles test Given the lag order of our model, zero is the only possible order for the complete set of common cycles $(C S C C)$. As suggested by Engle and Vahid (1993), the null of $C S C C-0$ (serial correlation common feature, in their terminology) can be tested by checking whether a pseudo structural form of the VECM encompasses the unrestricted reduced form of the VECM. The pseudo structural form is obtained by including, in the first $k$ equations, only the contemporaneous values of $n-k$ variables, leaving the remaining $n-k$ equations as in the unrestricted reduced form. The likelihood ratio test for the validity of the over-identifying restrictions is $\chi^{2}(12)=36.35$, with a p-value of [0.0003]. This suggests that the null of $C S C C-0$ can be rejected and that the identification of the structural innovations can be performed following the approach discussed in Warne (1993).

\subsection{Common trends analysis}

As mentioned in section 3, the presence of four cointegration relations between the six variables in the system implies that there are two distinct sources of shocks having permanent effects on some elements of $\mathbf{x}_{t}$. Based on the theoretical framework we interpret the permanent shocks as being a productivity shock $\left(\tau_{\theta}\right)$ and a nominal shock $\left(\tau_{\beta}\right)$. One identifying restriction imposed is a long-run monetary neutrality condition, i.e. we assume that the permanent nominal shock does not have a long-run impact on real GDP. Since this restriction ensures exact identification, it cannot be tested. However, finding long-run separation between real and nominal variables provides evidence in favour of its validity. Given the identification restrictions implied by the cointegration vectors, the former restriction is sufficient to ensure that the nominal permanent shock does not have a long-term impact on real money balances and the real stock market price index.

The common trends representation of the variables in levels is therefore the 
following

$$
\begin{aligned}
\left(\begin{array}{l}
y \\
f \\
m \\
i \\
l \\
\pi
\end{array}\right)_{t}=\left(\begin{array}{l}
y \\
f \\
m \\
i \\
l \\
\pi
\end{array}\right)_{0}+\left(\begin{array}{ll}
\gamma_{11} & 0 \\
\gamma_{21} & 0 \\
\gamma_{31} & 0 \\
\gamma_{41} & \gamma_{42} \\
\gamma_{51} & \gamma_{52} \\
\gamma_{61} & \gamma_{62}
\end{array}\right)\left(\begin{array}{l}
\tau_{\theta} \\
\tau_{\beta}
\end{array}\right)_{t} \\
+\Gamma^{*}(L)\left(\begin{array}{l}
\psi_{\theta} \\
\psi_{\beta} \\
v_{T S} \\
v_{S M} \\
v_{A D} \\
v_{F H}
\end{array}\right)_{t}
\end{aligned}
$$

where $v_{i} i=T S, S M, A D, F H$ are the transitory disturbances (uncorrelated with the permanent shocks) and the six exclusion restrictions imposed on the $\Gamma_{0}$ matrix are as follows

$$
\begin{aligned}
& \boldsymbol{\zeta}\left(\mathbf{Q}^{-1}\right)^{\prime}= {\left[\begin{array}{cccc}
0 & 0 & * & * \\
* & * & * & * \\
0 & * & * & * \\
* & * & * & * \\
* & * & * & * \\
0 & 0 & 0 & *
\end{array}\right] } \\
& \boldsymbol{v}_{t}=\left[\begin{array}{l}
v_{T S} \\
v_{S M} \\
v_{A D} \\
v_{F H}
\end{array}\right]
\end{aligned}
$$

These restrictions mean that: (i) the shock to the term structure does not have a contemporaneous impact on output, inflation and real balances; (ii) the liquidity preference shock does not have a contemporaneous impact on output and inflation; (iii) there is an underlying temporary shock to output (interpretable as a demand shock) that does not have a contemporaneous impact on inflation; (iv) the shock to the Fisher relation has a contemporaneous impact on all variables. The meaning of these identifying assumptions will be further discussed below. 


\subsubsection{Forecast error variance decomposition}

The estimated factor loading matrix $\boldsymbol{\Gamma}_{g}$ and the forecast error variance decomposition are reported in Table 4 and in Table 5 respectively. ${ }^{6}$ Notice that the real permanent shock has a negative, but not statistically significant, long-run impact on the nominal variables, explaining only $1 \%$ of the residual long-run variability (see Table 4, Panel A and Table 5, Panel C). It is therefore possible to interpret the nominal permanent shock as excess nominal money growth, by setting $\gamma_{41}=\gamma_{51}=\gamma_{61}=0$, as explained in the theoretical section.

Consider, for the sake of exposition, three dimensions of the forecast horizon: short-term (below one year), medium term (three years), and long-run (above five years). From the forecast error variance decompositions the following facts are worth noting.

First, depending on the forecast horizon there is a marked difference between the factors that explain variability. In the short- to medium-term, aggregate demand shocks are the main source of output variability (88\% at 1 year; $53 \%$ at 3 years) whilst productivity shocks are the main source of output variability in the long-run (about $70 \%$ already at 5 years). In the short-term, shocks to the Fisher relation are the main source of inflation variability ( $74 \%$ at 1 quarter) while the nominal permanent shock is the main source of inflation variability in the medium- to long-term (65\% at 3 years; $75 \%$ at 5 years). Thus, the forecast error variance decompositions of output and inflation illustrate some "textbook" features of our model: productivity driving trend output and aggregate demand shocks deviating output from the trend; inflation reflecting both dimensions of monetary policy (interest rates in the short-term; excess money growth in the long-term). Liquidity preference shocks are the most important determinant of real stock market price index volatility in the short-term (44\% at 1 quarter), whilst productivity shocks are the main source of stock market variability in the medium- to long-term ( $72 \%$ at 3 years; $78 \%$ at 5 years). In the short-term, shocks to the Fisher relation are the main source of real M3 variability (49\% at 1 quarter), while the productivity shock is the main source of real M3 variability in the medium- to long-term (43\% at 3 years; $73 \%$ at 5 years). However, in the short- to medium-term, aggregate demand and liquidity preference shocks are also important explanatory factors for real M3 variability. Short- and long-term

\footnotetext{
${ }^{6}$ The estimation was carried out using a Gauss code, which was written based on the CT routine in Rats by A. Warne and H. Hansen. Reported standard errors and confidence bands were produced by Monte Carlo simulation.
} 
interest rates seem to share the same underlying factors of variability displaying an interesting "reversal" in causal factors: in the short-term the productivity shock is the main source of variability $(42 \%$ and $33 \%$ at 1 quarter; short- and long-term interest rates respectively), whilst the nominal permanent shock is the main source of variability in the medium- to long-term $(60 \%$ and $74 \%$ at 3 years, for short- and long-term interest rates, respectively). Thus, the forecast error variance decompositions of interest rates illustrate another "textbook" feature of our model: due to "price-stickyness" short-term changes in interest rates are also changes in real interest rates and thus are to some extent related to productivity changes. In the long-run, nominal interest rates fully reflect anticipated inflation.

Second, depending on the type of variables there is a marked difference between the factors that explain variability: in the short-term the behaviour of real variables is more heterogeneous than the behaviour of nominal variables. In terms of forecast error variance decomposition the separation between the real and the nominal sides of the economy is apparent already at the 5 years horizon. In fact, at that horizon, the productivity shock is the main factor explaining variability of output, real stock prices and real M3 (69\%, 78\% and 73\%, respectively); in contrast, at that horizon, the permanent nominal shock is the main factor explaining variability of short- and long-term interest rates and inflation $(73 \%, 82 \%$ and $75 \%$, respectively). However, in the short-term, variability of real variables is explained by different factors: output by aggregate demand shocks, stock market by liquidity preference shocks, and real balances by Fisher equation shocks. On the nominal side, volatility of interest rates is explained mainly by the same shock (productivity). However, in the short-term, inflation variability is explained by a different factor (Fisher equation shock).

Our results are in line with previous findings of Rapach (2001), Dhar et al. (2000) and Lastrapes (1998). Rapach (2001) finds that productivity shocks are the main determinant of real US stock prices in the long-run and that nominal shocks (money supply shocks) explain about one third of real stock prices variance in the short run; Lastrapes (1998) and Dhar et al. (2000) find that money supply shocks are an important determinant of real stock prices in the short- and medium-term also for other major industrialised countries (G-7 and Holland). Furthermore, our results are coherent with the previous findings of King et al. (1991) and DeLoach and Rasche (1998) for the US economy, and Dhar et al. (2000) for the UK economy, suggesting that output fluctuations in the short term are explained neither by the (domestic) productivity trend nor by the (domestic) nominal trend. The results are also in line with the findings of Lee (1992) and Dhar et al. (2000), 
showing that productivity shocks explain little variation in US and UK inflation, and that inflation shocks have little explanatory power for real variables in the medium- to long-term.

\subsubsection{Impulse-response analysis}

Productivity and aggregate demand shocks The response of the economy to productivity and aggregate demand shocks, plotted against the forecast horizon, is illustrated in Figure 1 and Figure 5 respectively. One standard error $(68 \%)$ confidence intervals are also shown.

The real permanent shock (Figure 1) has permanent positive effects on real variables (output, the stock market, and real balances) and no long-run effects on nominal variables (interest rates and inflation).

The impact on real variables differs in their dynamics: the effect on the stock market peaks within four quarters $(+5.5 \%)$, whereas the impact on output peaks between five to six years $(+0.55 \%)$ and on real balances somewhat later (seven years; $+0.78 \%$ ). Furthermore, while the stock market displays overshooting, real balances and output gradually approach their respective new equilibrium values.

The positive productivity shock has a temporary deflationary impact on the economy and nominal interest rates and inflation temporarily fall below their equilibrium values.

The impact of the aggregate demand shock is illustrated in Figure 5. This shock can be interpreted as resulting, for example, from a fiscal expansion. Output, real M3, the stock market and both short-term and long-term interest rates increase showing a contemporaneous reaction to the aggregate demand shock; inflation starts increasing after two quarters. The impact of the shock on output, the stock market and the long term interest rate peaks within the quarter, and in one and two quarters respectively $(+0.4 \%,+2.3 \%,+17 \mathrm{bp})$, while the impact on inflation peaks within four quarters $(+0.1 \%)$, and the impact on the short term rate within three quarters $(+33 \mathrm{bp})$.

Monetary policy and financial shocks As explained in section 2 we interpret monetary policy as involving two dimensions: one, medium- to long-term, is the setting of the inflation objective reflected in the excess nominal money growth process $\left(\pi_{t}^{*} \Leftrightarrow \Delta^{2} m_{n, t}^{*}=\gamma_{2} \Delta \beta_{t}=\gamma_{2} v_{\beta, t}\right)$; another, short-term, is maintaining inflation on track by changing short-term interest rates, taking into account all shocks hitting the economy $(\Psi()$.$) . A third component is added \left(\varepsilon_{c b, t}\right)$ capturing 
non-systematic determinants of monetary policy. A crucial point in the identification of the latter component is that short-term real interest rates must (temporarily) increase in response to a (temporary) monetary policy tightening Thus, we interpret temporary deviations from the Fisher relation as capturing unexpected changes in the stance of monetary policy. The caveat is that the reaction of the economy to the Fisher equation shock may also capture other shocks.

The response of the economy to the permanent nominal shock (an increase in the long-run inflation objective of the monetary authorities) is illustrated in Figure 2: it has temporary effects on real variables (increasing output, the stock market, and real balances) and permanent effects on the nominal variables (increasing interest rates and inflation). This shock has a positive impact on real output that peaks within six quarters $(+0.13 \%)$ exhausting its expansionary impact within twenty six quarters; the shock has its largest impact on the stock market at the two quarters horizon $(+2.5 \%)$, having exhausted half of its effects between five and six quarters. Its positive impact on the stock market is completely exhausted within ten quarters. The permanent positive nominal shock has a long-run inflationary impact on the economy $(+0.4 \%)$ and nominal interest rates increase due to the Fisher effect $(+58 \mathrm{bp})$. However, real interest rates fall below equilibrium on impact. These results are consistent with the findings of Lastrapes (2001), who finds that, for some major industrialised countries, real liquidity effects are stronger than nominal liquidity effects. ${ }^{7}$

The reaction of the economy to the Fisher equation shock is shown in Figure 6. We interpret the response of the economy to this shock as the reaction to a surprise decline in the (real) short-term interest rate due to an unexpected (temporary) monetary policy loosening. In response to the cut in the short-term interest rate $(-14$ b.p. $)$ there is a positive impact on output $(+0.09 \%$; peaking at 7 quarters); there is a short-term "rally" in the bond and stock markets with a positive impact on real stock market prices $(+2.19 \%)$ and on bond prices $(-20$ b.p. in yields) peaking at two quarters.

Finally, note the similitude between the responses of the stock market, output

\footnotetext{
${ }^{7}$ These dynamic effects can be explained within a broad range of theoretical models. In particular, they are very similar to those implied by Blanchard (1981). When prices are sticky, an increase in the nominal money supply leads to an increase in real money balances. The increase in real balances and the expectation of higher future inflation lead to a temporary fall in real interest rates and to a stock market expansion. However, as soon as output responds to higher real stock prices, real interest rates start to increase, bringing back stock market prices to equilibrium. From the impulse response function it appears that the real liquidity effect lasts for just one quarter and output responds quickly to real stock market prices.
} 
and inflation to monetary policy loosening, irrespective of whether this is identified by the increase in the long-run inflation objective of the authorities or by the "surprise" reduction in the short-term interest rate (shock to the Fisher equation). Thus, in practice, economic agents may not be able to distinguish one shock from the other, i.e. distinguish temporary from permanent monetary policy tightening or loosening. This result illustrates the idea that central bank credibility is of utmost importance in understanding the response of the economy to monetary policy actions.

Systematic monetary policy response to shocks From a policy point of view another interesting exercise is to see how monetary policy reacts to the different structural shocks (dynamic reaction function), i.e. the $\Psi($.$) component$ in our theoretical framework. After all, there is a respected tradition in macroeconomics, starting with Poole (1970), linking the optimality of monetary policy rules (e.g. interest rate pegging versus flexible interest rates) with the type of shocks that hit the economy (financial versus real; temporary versus permanent) (see also Ireland (2000)). The response of the short-term interest rate (interpreted here as a dynamic reaction function) to the structural shocks can be analysed by looking at its response to the various shocks displayed in Figures 1, 3, 4 and 5.

Firstly, depending on the type of shock the dynamics of the short-term interest rate differs significantly. Consider first the supply and demand shocks (Figure 1 and Figure 5 respectively). In both cases the short-term interest rate deviates temporarily from the baseline (stochastic) trend. However, the direction of the reaction differs, declining in the case of the supply shock and increasing in the case of the demand shock. Both reactions are transitory even though one shock is permanent and the other is transitory. At the root of this result lies the fact that in both cases inflation deviates from the baseline (stochastic) trend only temporarily, decreasing in the first case and increasing in the second case (see Figure 1 and Figure 5). These reactions contrast with the case of the permanent nominal shock, where the short-term interest rate increases permanently (Figure 2). Thus, the reaction of the short-term interest rate seems to contribute to stabilizing the economy: alleviating the temporary deflationary pressures associated with positive productivity shocks and counteracting the inflationary pressures in the other two cases.

Secondly, note the reaction of the short-term interest rate when the economy faces a term structure shock (Figure 3). This shock can be interpreted as a "financial disturbance" affecting the stock and bond markets negatively: the (real) 
long-term interest rate increases and the stock market "crashes". The short-term interest rate declines temporarily alleviating the deflationary impact of the shock. In fact, output does not seem to be significantly affected by the disturbance.

Thirdly, note the (no) reaction of the short-term interest rate when the economy faces a liquidity preference shock (Figure 4). This shock can be interpreted as a "liquidity" preference shock affecting the stock market negatively but without affecting the bond market significantly (Figure 4). There seems to be substantial interest rate smoothing by the monetary authorities. This is reminiscent of the famous Poole (1970) recommendation of interest rate pegging to stabilize output when facing shocks to the LM curve: in fact, output does not seem to be significantly affected.

\subsection{A closer look at cyclical dynamics}

In this section we concentrate our attention on cyclical dynamics, which can be justified on the grounds that it is closer to the traditional object of business cycle inquiry, i.e. modelling "detrended" economic variables. After looking at the main characteristics of the overall cycle, two historical decompositions exercises are carried out in order to identify the likely factors underlying the cyclical dynamics of the euro area economy in the 1986:Q1-2000:Q4 period. ${ }^{8}$ One exercise decomposes the cycle into equilibrium and disequilibrium dynamics; another decomposes the cycle into the contributions of its underlying determinants (structural shocks).

The estimated cyclical components for the six variables in the system are shown in Figure 7. The cyclical component of the short-term interest rate is plotted jointly with all the other cyclical components. Additionally, the last panel (on the right hand side at the bottom) shows the cyclical components of the stock market and inflation plotted jointly. Table 6 reports the correlation matrix of the overall cyclical components.

The cyclical components are all positively correlated except the stock market cycle which is negatively correlated with the other cycles. The highest contemporaneous correlation is between the cyclical components of output and real balances, followed by the relatively high correlations (in absolute value) between stock market and real balances cyclical components and between the stock market cycle and the long-term interest rate cycle. Also noticeable is the high positive correlation between the short-term interest rate cycle and the output cycle. The correlations

\footnotetext{
${ }^{8}$ Some experimentation showed that using twenty lags in the historical decomposition was sufficients to achieve a full reconstruction of the cyclical components.
} 
seem to have been stable in the sample period.

\subsubsection{Historical decompositions}

Equilibrium and disequilibrium dynamics Historical decompositions into dynamics towards the attractor (DTA; disequilibrium dynamics) and dynamics along the attractor (DAA; equilibrium dynamics) are shown in Figure 8. Table 6 (Panel B) and Table 7 (Panel A) report, respectively, the correlation matrices and the proportion of the variance of each cycle explained by the two components.

An interesting result is the change in the magnitude of the correlation between the cyclical components of the short-term interest rate and inflation: the correlation of the DAA is much stronger than that of the DTA. To understand this result recall that both permanent shocks imply a short-run positive correlation between inflation and (nominal) interest rates (Figure 1 and Figure 2). However, the transitory Fisher equation shock, which we interpret as capturing monetary policy "surprises", implies a negative short-run correlation between inflation and the short-term interest rate (Figure 6). It is this effect that explains the lower correlation in the DTA, which nevertheless remains positive due to the effect of aggregate demand shocks on both variables (Figure 5). Note that the correlation of inflation and long-term interest rate changes sign because the impact of aggregate demand shocks is less important to explain the variability of the long-term interest rate than of the short-term interest rate (Table 5, Panels A and B).

One conclusion that can be drawn is that monetary policy was countercyclical, also given the high positive contemporaneous correlation between the DTA of short-term interest rate and output (and real M3 balances). In this sense the negative correlation between the inflation and the stock market cycles (Figure 7; right hand side panel at the bottom) can also be explained by the countercyclical nature of monetary policy.

There are two distinct phases of the output cycle in 1986:Q1-2000:Q4: an expansionary phase, from 1986 until 1992, and a contractionary phase, from 1993 until 2000. Given the high contemporaneous correlations of all cyclical components the same classification applies to the other variables (the stock market cycle has an opposite sign). As illustrated in Figure 8 both the DAA and the DTA were important factors in explaining cyclical dynamics of all variables (see also Table 7, Panel A). However, the DTA played a more important role in the expansionary phase, particularly in 1989-1991, whereas the DAA palyed a greater role in the contractionary phase, especially in 1999-2000. Thus, one can conclude 
that disequilibrium factors (temporary shocks) were the most important determinants of cyclical dynamics in the euro area in the late 1980s early 1990s, whereas equilibrium factors (permanent shocks) were the most important determinants of cyclical dynamics in the euro area in the late 1990s and 2000.

Underlying structural shocks The historical decompositions of the cyclical dynamics of the euro area economy into their underlying determinants (structural shocks) are shown in Figures 9 to 14. Table 7 (Panel B) reports the proportion of the variance of each component (DAA and DTA) explained by each structural shock. The following remarks can be made for the 1986:Q1-2000:Q4 period.

Firstly, the productivity shock explains the bulk of the DAA for all variables. Nevertheless, the nominal permanent shock contributed to the dynamics of interest rates and the stock market, as well. Secondly, the aggregate demand shock explains the bulk of the DTA for all variables except inflation, which was largely explained by the Fisher parity shock. This shock was important also for the other variables, except output. Thirdly, all transitory shocks were relevant for the DTA of the stock market. Fourthly, the term structure shock was an important factor for the DTA of interest rates and the stock market but not for other variables. Overall, the findings are largely coherent with the short-run predictions of the model.

Taken together these results suggest that the term structure shock, the aggregate demand shock, and the Fisher parity shock were important sources of non-separability in the short-run.

Confirming the results from the decomposition into DAA and DTA the aggregate demand shock played a more important role in the expansionary phase, particularly in 1989-1991, whereas the productivity shock played a greater role in the contractionary phase, especially in 1999-2000 (see Figures 9 to 14). Thus, one can conclude that the most important determinants of cyclical dynamics in the euro area were demand shocks in the late 1980s early 1990s, and supply shocks in the late 1990 s and 2000.

\subsection{A closer look at the stock market}

In this section we take a closer look at the dynamics of the stock market. Firstly, we discuss whether and how our framework helps in understanding the inflation hedging proprieties of the stock market, which is a much discussed topic in the 
finance literature. Secondly, we discuss the issue of measuring stock market price misalignment and its likely underlying determinants, which has been the recent focus of attention both in academic and public discussions.

Inflation and stock market returns Our theoretical framework suggests that the stock market provides, in the long-run, a good hedge against inflation: the cointegration relationship between output and the stock market price index suggests that stock market returns are linked to the underlying productivity growth of the economy, ensuring a positive (risk adjusted) real return in the long-run. This proposition is best illustrated in Figure 1: in response to the productivity shock the real stock market price index increases permanently whereas inflation does not change. However, the short-run dynamics of inflation and stock market returns might suggest different hedging proprieties: in the first four quarters after the productivity shock inflation is decelerating, a movement that is accompanied by an increasing real stock market price index. This implies a (short-run) negative correlation between inflation and stock market returns creating the illusion that the stock market has poor hedging proprieties against inflation.

Consider the response to the permanent nominal shock: in the long-run the real stock market price index does not change, whereas inflation increases permanently. This suggests no long-run correlation between inflation and stock market returns. However, the short-run dynamics of inflation and stock market returns might suggest good hedging proprieties: in the first two quarters after the nominal shock, both inflation and the real stock market price index are increasing implying a (short-run) positive correlation.

The conclusions are that the hedging proprieties of the stock market against inflation depend on the horizon considered and are difficult to determine ex-ante, given that they depend on the type of shocks hitting the economy. Forecast error variance decomposition suggests that it is the permanent real shock (see Table 5) and the temporary liquidity preference shock that explain the stock market in the short-run. These shocks tend to produce a (short-run) negative correlation between inflation and stock market returns, creating the illusion that the stock market has poor hedging proprieties against inflation in the euro area (see Figure 1 and Figure 4). Thus, our analysis provides an alternative interpretation for this empirical regularity, which can also be explained, in theory, by a countercyclical monetary policy (see Bakshi and Chen (1996)). 
The stock market cycle: the role of financial and productivity shocks

Even though stock price misalignments are transitory phenomena, they may impose large costs on the economy. Therefore, it seems desirable to know whether, at a given point in time, stock market prices are consistent with their equilibrium values. The fact that stock prices may follow dynamics that imply unrealistic expectations concerning future dividends has been rationalised in the literature by the concept of rational bubbles (Diba and Grossman, 1983; Flood and Garber, 1980; Blanchard, 1979). Furthermore, Froot and Obstfeld (1991) and Ikeda and Shibata (1992) developed models in which stock market price bubbles are determined partially or completely by fundamentals (intrinsic bubble). Thus, differently from the concept of extrinsic bubbles, the bubble process can be explained by self-fulfilling expectations about fundamentals, as well. In this framework, the distinction between fundamental and non-fundamental price dynamics is not meaningful. Rather, it is the distinction between permanent and transitory fundamental dynamics that may provide information concerning the degree of misalignment in stock market prices. The cyclical real asset price component, together with other traditional indicators, could, in fact, provide useful information for macroeconomic policy.

According to the theoretical model, it is possible to relate the long-run evolution of dividends to productivity, so that the decomposition of the stock market index into trend and cyclical components is suitable of economic interpretation. The strong correlation of the DTA and DAA of the stock market index with the cyclical dynamics of all of the other series suggests that transitory fluctuations in stock market prices and macroeconomic variables are related. The historical decomposition of the stock market cycle in the euro area in the 1986:Q1-2000:Q4 period, ilustrated in Figure 10, allows a number of interesting conclusions.

Firstly, the stock market cycle can be explained to a large extent by productivity shocks. This result is consistent with intrinsic bubble theory since stock market prices were related to its fundamental determinants also over the cycle.

Secondly, productivity shocks do not fully account for the behaviour of the stock market over the cycle. Of particular interest is the comparison between the determinants of the stock market cycle at the end of the 1980s and the end of the 1990s. In both cases overshooting following productivity shocks played a role. However, liquidity preference shocks played a dominant role in the 1980s. In the 1990s, monetary policy shocks seem to have had an important role in explaining the stock market cycle.

Finally, aggregate demand shocks, which played an important role until the 
mid-1990s, did not contribute to the more recent dynamics of the stock market in the euro area.

\section{Implications for monetary policy}

In this section we try to answer the question of whether price stability oriented monetary policy is consistent with financial stability in the euro area. We consider that price stability and financial stability are mutually consistent (medium- to long-run) objectives if the former aim is achieved without affecting the (stochastic) trend in the real stock market price index and if its cyclical developments are not amplified. We first review the empirical evidence presented in section 4 drawing here the relevant policy conclusions. Next, using the SVAR, we generate artificial euro area data under a price stability environment and compare it with the actual data. The latter exercise allows computing the costs and benefits of such regime evaluating the consistency between price stability and financial stability.

\subsection{Price stability and financial stability: consistency suggested by em- pirical evidence}

The forecast error variance decompositions and impulse-response analysis presented in section 4 suggest that price stability and financial stability are mutually consistent objectives in the euro area. In fact, according to our empirical analysis, long-run separation between the real and the nominal sides of the economy seems to prevail in the euro area. Additionally there is empirical support for interpreting the nominal trend as excess nominal growth of money process, reflecting the medium- to long-run inflation objective of the monetary authorities. Thus, these facts suggest that, on the one hand, the (stochastic) trend in the real stock market price index is not affected by changing to a price stability environment (Figure 2; Table 5) and, on the other hand, successfully implementing such policy would shut-off one source of short-term volatility in the stock market.

A related but somewhat different question is whether monetary policy should focus directly on, or give more prominence to, stock market developments. The analysis presented in section 4 suggest that this might be undesirable and difficult or even impossible to achieve in the euro area. Firstly, forecast error variance decompositions and impulse-response analysis suggest that the bulk of stock market volatility (variance) is due to overshooting in response to permanent productivity shocks and also to temporary liquidity preference shocks (Figure 1 and Figure 4, 
respectively; Table 5). Thus, monetary policy cannot prevent most short-term real stock market price volatility. Secondly, direct reaction to stock market price developments may lead to undesirable results. Consider, for example, a scenario in which improvements in productivity generate a rise in stock market fundamentals, as well as an increase in potential output. Responding to the rise in equity prices with a monetary policy tightening has the undesirable effect of temporarily stifling the beneficial impact of the technology boom unless the productivity shock is inflationary, which seems unlikely from a theoretical point of view and is against our empirical findings (see also Smets, 1997).

\subsection{Price stability and financial stability: consistency suggested by a simulation exercise}

To further investigate the consistency between price stability and financial stability we use the estimated SVAR to generate artificial euro area data under a price stability environment and compare it with the actual data. As suggested by Bagliano and Morana (1999a,b,c,) and Bagliano et al. (2001a,b) we consider the Beveridge-Nelson-Stock-Watson permanent component of inflation as the core inflation process. This definition has also been recently advocated by Cogley and Sargent (2001). ${ }^{9}$ The simulation exercise is performed under the simplifying assumption that the monetary authority aims at keeping core inflation at a level consistent with price stability. This assumption captures the idea that not all price developments can be avoided by the monetary authorities, namely temporary price developments resulting from productivity shocks. In our model, given long-run separation between the real and the nominal sides of the economy, the core inflation process $\left(\pi_{t}^{c}\right)$ is defined as

$$
\pi_{t}^{c}=\pi_{0}+\mu_{\pi} t+\gamma_{6,2} \sum_{j=0}^{t-1} \psi_{\beta, t-j},
$$

where: $\pi_{t}^{c}, \pi_{0}, \mu_{\pi}, \gamma_{6,2}$, and $\psi_{\beta, t-j}$ are the relevant elements in the matrices defined in equation (3.4).

\footnotetext{
${ }^{9}$ Other works in which this definition of core inflation has been suggested are Blix (1997) and Dhar et al. (2000). Bagliano and Morana (1999a,b) provide evidence that allowing for cointegration restrictions and a larger information set may lead to core inflation estimates which are more in line with the expected theoretical properties of a core inflation process.
} 
Controlling inflation in the SVAR To carry out the policy simulations we must first derive a monetary policy rule for controlling inflation in the SVECM. ${ }^{10}$ Consider that at a certain point in time $(t)$ there is a positive deviation of core inflation from the price stability objective $\left(\pi_{t}^{c}-\pi^{*}>0\right)$. Without lack of generality, suppose that the monetary autority wants to bring the economy onto the aimed price path next period, i.e. at time $(t+1)$. The necessary innovation in core inflation is given by

$$
\begin{aligned}
\psi_{\beta, t+1} & =\left(\pi^{*}-\pi_{0}-\mu_{\pi}(t+1)-\gamma_{6,2} \sum_{j=0}^{t-1} \psi_{\beta, t-j}\right) / \gamma_{6,2} \\
& =\left(\pi^{*}-\pi_{t}^{c}-\mu_{\pi}\right) / \gamma_{6,2} .
\end{aligned}
$$

From equation (3.2) and taking equation (3.14) into account it can be shown that the short-term interest rate innovation necessary to ensuring the correction in core inflation is given by

$$
b_{F H}(L) y_{F H, t+1}=\theta_{F H}+\Lambda_{F H} \boldsymbol{\varphi}_{t+1},
$$

where: $b_{F H}(L), y_{F H, t+1}, \theta_{F H}, \boldsymbol{\Lambda}_{F H}, \boldsymbol{\varphi}_{t+1}$ are the relevant entries in the Fisher parity equation in the RVAR representation of the model. The feasibility condition for controlling inflation in the SVAR depends on the impact of the policy innovation on the Fisher parity disequilibrium given by

$$
\frac{\partial y_{F H, t+1}}{\partial \psi_{\beta, t+1}}=\boldsymbol{\Lambda}_{F H, \beta},
$$

which must have the "right" sign and be statistically significant. ${ }^{11}$

\footnotetext{
${ }^{10}$ Johansen and Juselius (2001) derived a control rule for the VECM representation that could, in principle, be used for simulating euro area data under a regime of price stability. However, the feasibility condition for controlling inflation in the VECM representation is based on the long-run response of inflation to interest rate innovations given by the relevant entry in the longrun impact matrix in the Wold VMA representation $(\mathrm{C}(1)$ matrix). Since the latter, in general, cannot be identified with orthogonal innovations to the interest rate equation, its sign and significance reflect both endogenous and exogenous responses of interest rates to macroeconomic developments and, thus, its use is not appropriate for policy simulation exercises. However, the alternative methodology that we propose is not immune to the Lucas critique: our structural parameters may not be policy invariant.

${ }^{11}$ By "right" sign we mean that the real short-term interest rate must increase for a monetary policy innovation to trigger a disinflationary process.
} 
The required short-term interest rate level necessary to achieve price stability in the next period (new policy rule) is given by

$$
i_{t+1}=i_{t}+\boldsymbol{\Lambda}_{F H, \beta} \psi_{\beta, t+1} .
$$

If desirable, core inflation correction can be achieved at a longer horizon by adjusting the short-term interest rate in smaller successive steps.

Policy simulations We start with an unconditional control exercise applied to the 1987:Q1-2000:Q4 period. In Figure 15 (upper panel) the observed shortterm interest rate path is compared with simulated series that correspond to the short-term interest rate levels that would have had to be implemented at each point in time to achieve respectively $2 \%, 1.5 \%$ and $1 \%$ inflation, but assuming that the rule was never followed. The lower panel shows the underlying simulated core inflation processes.

As expected, the observed interest rate series is, on average, lower than the levels that would have been consistent with the aim of achieving inflation rates below $2 \%$. Note that since mid-1996, the short-term interest rate has been set at a level that broadly aims at increases in the GDP deflator between $1.5 \%$ and $2 \%$ in the euro area.

The simulation also suggests that the recent deviation of inflation from the definition of price stability (Figure 15; lower panel) is not related to the interest rate setting but instead might be due to the temporary impact on inflation of productivity shocks (supported by the historical decompositions in Figure 14).

Next we simulate euro area data assuming that the control rule is actually implemented starting from 1987:Q1. The euro area economy is exposed to historical shocks (except the short-term interest rate) to obtain the time path of the variables under the new regime. We considered $2 \%, 1.5 \%$ and $1 \%$ inflation objectives. In Figure 16 we plot the simulated and estimated cyclical components for output and the stock market, under the different price stability scenarios. From the plots it can be noticed that a successful inflation control policy would have generated a real stock market price index closer to their trend value, without avoiding cyclical fluctuations. In addition, the output costs of price stability, relative to the realised output cycle, tend to zero on average over the sample considered, since the initial negative impact on the output gap in the late 1980s is compensated by a positive impact in the 1990s. The results are robust to the three levels of the inflation objective considered. 
In summary, the control exercise confirms the main results of the empirical analysis: a monetary policy committed to price stability has a beneficial impact on financial stability but cannot avoid the large stock market fluctuations observed empirically.

\section{Conclusions}

The interest rate and the asset price channels seem to play an important role in the transmission mechanism in the euro area as real output corrects relative to the slope of the yield curve and stock market disequilibrium.

Permanent productivity shocks drive the stock market in the long-run and explain the tendential behaviour of stock market price over the cycle. However, the cyclical dynamics in the stock market are also explained by transitory shocks, particularly preference to liquidity shocks (which capture the speculative nature of the market), aggregate demand shocks, and monetary policy shocks.

Permanent monetary surprises have a strong, yet temporary, impact on the stock market and a permanent impact on inflation. This suggests that a monetary policy focused on the stock market could easily become incompatible with the price stability objective. On the contrary, a price stability oriented monetary policy may have a beneficial impact also on the stock market. Nevertheless, it is unlikely that stock market price fluctuations can be controlled or avoided through monetary policy actions.

\section{References}

[1] Alchian, A.A. and B. Klein, 1973, On a Correct Measure of Inflation, Journal of Money Credit and Banking, 5(1), 173-91.

[2] Bagliano, F.C. and C. Morana, 1999a, A Common Trends Model of UK Core Inflation, Empirical Economics, forthcoming.

[3] Bagliano, F.C. and C. Morana, 1999b, Measuring US Core Inflation, Journal of Macroeconomics, forthcoming.

[4] Bagliano, F.C. and C. Morana, 1999c, Measuring Core Inflation in Italy, Giornale degli Economisti e Annali di Economia, 58(3-4), 301-28. 
[5] Bagliano, F.C., R. Golinelli and C. Morana, 2001a, Core Inflation in the Euro Area, Applied Economics Letters, forthcoming.

[6] Bagliano, F.C., R. Golinelli and C. Morana, 2001b, Inflation Modelling in the Euro Area, mimeo.

[7] Bakshi, G. S. and Z. Chen, 1996, Inflation, Asset Prices, and the Term Structure of Interest Rates in Monetary Economies, Review of Financial Studies, 9, 241-275.

[8] Bernanke, B. and M. Gertler, 1989, Agency Costs, Net Worth, and Business Fluctuations, The American Economic Review, 79(1), 14-31.

[9] Bernanke, B. and M. Gertler, 1999, Monetary Policy and Asset Price Volatility, New Challenges for Monetary Policy, Federal Reserve of Kansas City, $17-51$.

[10] Bernanke, B., M. Gertler and S. Gilchrist, 1998, The Financial Accelerator in a Quantitative Business Cycle Framework, NBER 6455.

[11] Blanchard, O.J., 1979, Speculative Bubbles, Crashes and Rational Expectations, Economics Letters, 3, 387-89.

[12] Blanchard, O.J., 1981, Output, the Stock Market, and Interest Rates, American Economic Review, 71, 132-143.

[13] Blix, M., 1997, Underlying Inflation: A Common Trends Approach, Sverige RiksBank Working Paper Series, no. 23.

[14] Bondt de, G., 1999, Credit Channels and Consumption in Europe: Empirical Evidence, BIS Working Papers, no. 69.

[15] Boone, L., C. Giorno and P. Richardson, 1998, Stock Market Fluctuations and Consumption Behaviour: Some Recent Evidence, OECD, Economics Department, Working Papers, no. 208.

[16] Borio C.E.V., N. Kennedy and S.D. Prowse, 1994, Exploring Aggregate Asset Price Fluctuations Across Countries. Measurement, Determinants and Monetary Policy Implications, BIS Economic Papers, no. 40.

[17] Brand, C. and N. Cassola, 2000, A Money Demand System for Euro Area M3, European Central Bank Working Papers Series, no.39. 
[18] Cecchetti S.G., H. Genberg, J. Lipsky, and S. Wadhwani, 2000, Asset Prices and Central Bank Policy, Geneva Reports on the World Economy, no. 2.

[19] Coenen G. and J.L.Vega, 1999, The Demand for M3 in the Euro Area, European Central Bank Working Papers Series, no.6.

[20] Cogley, T., 1999, Should the Fed Take Deliberate Steps to Deflate Asset Price Bubbles?, Federal Reserve Bank of San Francisco Economic Review, 1, 42-51.

[21] Cogley, T. and T.J. Sargent, 2000, Evolving Post-World II U.S. Inflation Dynamics, mimeo, Stanford University.

[22] Davidson, J., 1998, Structural Relations, Cointegration and Identification: Some Simple Results and their Application, Journal of Econometrics, 87, 87-113.

[23] Dhar, S., D. Pain and R. Thomas, 2000, A Small Structural Empirical Model of the UK Monetary Transmission Mechanism, Bank of England Working Paper Series, no. 113.

[24] Diba, B.T. and H.I. Grossman, 1983, Rational Asset Price Bubbles, NBER working paper no.1059.

[25] DeLoach, S.B. and Rasche, R.B., 1998, Stochastic Trends and Economic Fluctuations in a Large Open Economy, Journal of International Money and Finance, 17, 565-96.

[26] Engle, R.F. and F. Vahid, 1993, Common Trends and Common Cycles, Journal of Applied Econometrics, 8, 341-60.

[27] Filardo A.J., 2000, Monetary Policy and Asset Prices, Federal Reserve of Kansas City Economic Review, 3, 11-37.

[28] Flood, R.P. and P. Garber, 1980, Market Fundamentals Versus Price Level Bubbles: The First Tests, Journal of Political Economy, 88, 745-70.

[29] Friedman, Milton, 1988, Money and the Stock Market, Journal of Political Economy, 96, 221-245.

[30] Froot, K. and M. Obstefeld, 1991, Intrinsic Bubbles: the Case of Stock Prices, The American Economic Review, 81(5), 1189-1214. 
[31] Gallo, G.M. and H. Kempf, 1995, Cointegration, Codependence and Economic Fluctuations, European University Institute, Working Paper in Economics, no. 95/27.

[32] Goodhart, C.A., 1999, Time, Inflation and Asset Prices, mimeo, London School of Economics.

[33] Goodhart, C.A. and B. Hofmann, 2000a, Financial Variables and the Conduct of Monetary Policy, Sverige Riksbank, Working Paper Series, no.112.

[34] Goodhart, C.A. and B. Hofmann, 2000b, Do Asset Prices Help to Predict Consumer Price Inflation, The Manchester School, 68, Supplement, 122-140.

[35] Golinelli, R. and S. Pastorello, 2000, Modelling the Demand for M3 in the Euro Area, manuscript, University of Bologna.

[36] Gordon, M.J., 1962, The Investment, Financing and Valuation of the Corporation, Homewood, Richard D. Irwin.

[37] Granger, C.W.J. and N. Haldrup, 1997, Separation in Cointegrated Systems and Persistent-Transitory Decompositions, Oxford Bulletin of Economics and Statistics, 59(4), 449-63.

[38] Hamilton, J.D. and C.H. Whiteman, 1985, The Observable Implications of Self Fulfilling Expectations, Journal of Monetary Economics, 19, 145-69.

[39] Ikeda, S. and A. Shibata, 1992, Fundamentals-Dependent Bubbles in Stock Prices, Journal of Monetary Economics, 30, 143-68.

[40] Ireland, Peter N., 2000, Interest Rates, Inflation, and Federal Reserve Policy since 1980, Journal of Money, Credit and Banking, 32, 3(1), 417-434.

[41] Johansen, S., 1988, Statistical Analysis of Cointegrating Vectors, Journal of Economic Dynamics and Control, 12, 231-54.

[42] Johansen, S. and K. Juselius, 2001, Controlling Inflation in a Cointegrated Vector Autoregressive Model with Application to US Data, European University Institute, mimeo.

[43] King R.G., Plosser C.I., Stock J.H., and Watson M.W., 1991, Stochastic Trends and Economic Fluctuations, The American Economic Review, 81(4), 819-40. 
[44] Kiyotaki N., and J. Moore, 1997, Credit Cycles, Journal of Political Economy, 105, 211-48.

[45] Konishi, T. and C.W.J. Granger, 1992, Separation in Cointegrated Systems, mimeo, University of California, San Diego.

[46] Lastrapes, W.D., 1998, International Evidence on Equity Prices, Interest Rates and Money, Journal of International Money and Finance, 17, 377-406.

[47] Lee, B.-S., 1992, Causal Relation Among Stock Returns, Interest Rates, Real Activity, and Inflation, The Journal of Finance, XLVII (4), 1591-1603.

[48] Ludvigson, S. and C. Steindel, 1999, Federal Reserve Bank of New York, July, 29-51.

[49] Mellander, E., A. Vredin and A. Warne, 1992, Stochastic Trends and Economic Fluctuations in a Small Open Economy, Journal of Applied Econometrics, 7, 369-94.

[50] Modigliani, F., 1971, Monetary Policy and Consumption, in Federal Reserve Bank of Boston, Consumer Spending and Monetary Policy: the Linkages.

[51] Peersman, G. and F. Smets, 2000, The Industry Effects of Monetary Policy in the Euro Area, mimeo, European Central Bank.

[52] Poole, William, 1970, Optimal Choice of Monetary Policy Instruments in a Simple Stochastic Macro Model, Quarterly Journal of Economics, 84, 197216.

[53] Poterba, J.M., 2000, Stock Market Wealth and Consumption, Journal of Economic Perspectives, 14(2), 99-118.

[54] Proietti, T. , 1997, Short-Run Dynamics in Cointegrated Systems, Oxford Bulletin of Economics and Statistics, 59, 3, 403-422.

[55] Rapach, D.E., 2001, Macro Shocks and Real Stock Prices, Journal of Economics and Business, 53, 5-26.

[56] Sellin, P., 2001, Monetary Policy and the Stock Market: Theory and Empirical Evidence, Journal of Economic Surveys, 15, 4, 491-541. 
[57] Smets, F., 1997, Financial Asset Prices and Monetary Policy: Theory and Evidence, CEPR Working Paper, no. 1751.

[58] Stock, J.H. and M.W. Watson, 1988, Testing for Common Trends, Journal of the American Statistical Association, 83, 1097-107.

[59] Tobin, J., 1969, A General Equilibrium Approach to Monetary Theory, Journal of Money, Credit, and Banking, 1, 15-29.

[60] Vermeulen, P., Business Fixed Investment: Evidence of Financial Accelerator in Europe, European Central Bank Working Paper Series, no. 37.

[61] Vlaar, P.J.G and H. Schuberth, 1998, Monetary Transmission and Controllability of Money in Europe: a Structural Vector Error Correction Approach, De Nederlandsche Bank Staff Reports, no.36.

[62] Warne, A., 1993, A Common Trends Model: Identification, Estimation and Inference, Seminar Paper No. 555, IIES, Stockholm University. 


\section{Appendix: the theoretical model}

The economy is represented by six endogenous variables measured in natural logarithms: real GDP $\left(y_{t}\right)$, inflation defined as, $p_{t}-p_{t-1}=\pi_{t}$ where $p_{t}$ is the GDP deflator, real M3 balances $\left(m_{t}-p_{t}=r m_{t}\right)$, short-term interest rate $\left(i_{t}\right)$, bond yield $\left(l_{t}\right)$, and real stock market price index $\left(f_{t}\right)$ which is the log of the stock market price index deflated by the GDP deflator.

\subsection{The steady-state of the model}

Fundamental stochastic processes Two underlying stochastic processes drive the endogenous variables in the long-run, namely a technology variable $\left(\theta_{t}\right)$ and a nominal variable $\left(\beta_{t}\right)$ related to the long-run inflation objective of the monetary authorities. These variables evolve over time according to the laws of motion

$$
\begin{gathered}
\theta_{t}=k_{\theta}+\theta_{t-1}+v_{\theta, t} \\
\beta_{t}=\beta_{t-1}+v_{\beta, t},
\end{gathered}
$$

where $v_{\theta, t}$ and $v_{\beta, t}$ are uncorrelated white noise processes and $k_{\theta}>0$ is a parameter. Thus, $\theta_{t}$ and $\beta_{t}$ are $I(1)$ processes.

Aggregate supply The supply side of the economy (AS) is described by

$$
\pi_{t}=\pi_{t}^{*}+\alpha_{1}\left(y_{t}-y_{t}^{*}\right)
$$

where expected inflation $\left(\pi_{t}^{*}\right)$ and potential output $\left(y_{t}^{*}\right)$, defined as $\lim _{h \rightarrow \infty} E_{t}\left(\pi_{t+h}\right)=$ $\pi_{t}^{*}$ and $\lim _{h \rightarrow \infty} E_{t}\left(y_{t+h}\right)=y_{t}^{*}$, evolve over time according to

$$
\begin{gathered}
y_{t}^{*}=\gamma_{1} \theta_{t} \\
\pi_{t}^{*}=\gamma_{2} \beta_{t}+\gamma_{3} \theta_{t},
\end{gathered}
$$

and where $\alpha_{1} \geq 0, \gamma_{1} \geq 0, \gamma_{2} \geq 0, \gamma_{3} \geq 0$ are parameters.

Apart from the supply equation our model builds on portfolio balance and arbitrage relations. 
Money demand Based on portfolio theory, money demand can be specified as a function of a scale factor and a vector of relative rates of return

$$
r m_{t}=\phi_{1} y_{t}-\phi / \mathbf{s}_{t},
$$

where $\mathbf{s}_{t}$ is a vector of spreads of yields on alternative assets with the (own) rate of return on real balances and $\phi$ is a vector of parameters. As the model includes four assets (M3, short-term bills, long-term bonds and stocks), we assume that there is a stationary combination of three yield spreads ${ }^{12}$ such that

$$
\phi / \mathbf{s}_{t}=\varepsilon_{m, t} \sim I(0),
$$

where

$$
\varepsilon_{m, t}=\rho_{1} \varepsilon_{m, t-1}+v_{s, t} \quad \rho_{1}<1 .
$$

and $v_{s, t}$ is white noise.

Term structure One arbitrage relationship links the yields on short-term bills and long-term bonds

$$
l_{t}=i_{t}+\phi_{l}+\varepsilon_{l, t},
$$

where $\varepsilon_{l, t}$ follows

$$
\varepsilon_{l, t}=\rho_{2} \varepsilon_{l, t-1}+v_{l, t} \quad \rho_{2}<1
$$

and $v_{l, t}$ is white noise.

The expectations theory of the term structure of interest rates can be expressed in logarithmic form as

$$
l_{t}=\frac{1}{n} \sum_{j=0}^{n-1} E_{t}\left[i_{t+j}\right]+\phi_{l},
$$

where $l_{t}$ is expressed as an average of expected one period log yields, $E_{t}\left[i_{t+j}\right], \phi_{l}$ is a term premium and $n$ is the maturity of the bond. Subtracting $i_{t}$ from both sides we have the relationship in (7.7)

$$
l_{t}-i_{t}=\phi_{l}+\frac{1}{n} \sum_{j=0}^{n-1} E_{t}\left[i_{t+j}-i_{t}\right],
$$

with $\frac{1}{n} \sum_{j=0}^{n-1} E_{t}\left[i_{t+j}-i_{t}\right]=\varepsilon_{l, t}$.

\footnotetext{
${ }^{12}$ The validity of the assumption on the existence of a stationary combination of three yield spreads is tested against the data as explained in section 4.1.
} 
Fisher parity Another arbitrage relationship links the rate of return on capital with the short-term interest rate

$$
i_{t}=\phi_{f p}+\phi_{3} \pi_{t}+\varepsilon_{i, t}
$$

where $\phi_{f p}$ is the sum of the real short-term interest rate and the inflation risk premium, and $\varepsilon_{i, t}$ is a stationary disturbance

$$
\varepsilon_{i, t}=\rho_{3} \varepsilon_{i, t-1}+v_{i, t} \quad \rho_{3}<1 .
$$

By writing the real rate of return on capital as

$$
1+\rho_{t}=(1+\rho) \exp \left(\varepsilon_{\rho, t}\right)
$$

the Fisher hypothesis for the short-term interest rate can be expressed as

$$
1+R_{t}=\left(1+\rho_{t+1}\right)\left(1+\pi_{t+1}^{*}\right) \exp \left(\varepsilon_{r p, t+1}\right),
$$

where $R_{t}$ is the gross nominal return on a short-term investment, from $t$ to $t+1$, $\varepsilon_{r p, t}$ is a stationary risk premium

$$
\varepsilon_{r p, t}=\rho_{4} \varepsilon_{r p, t-1}+v_{r p, t} \quad \rho_{4}<1 .
$$

Expected inflation is given by

$$
\left(1+\pi_{t}^{*}\right)=\left(1+\pi_{t}\right) \exp \left(v_{\pi^{*}, t}\right)
$$

where $v_{\pi^{*}, t}$ is a stationary process. By combining the above equations one obtains

$$
1+R_{t}=(1+\rho)\left(1+\pi_{t+1}\right) \exp \left(\varepsilon_{r p, t+1}+\varepsilon_{\rho, t+1}+v_{\pi^{*}, t+1}\right),
$$

which yields the log linear approximation

$$
i_{t}=\left(\rho+\varepsilon_{r p, t+1}\right)+\pi_{t+1}+\left(\varepsilon_{\rho, t+1}+v_{\pi^{*}, t+1}\right),
$$

leading to (7.8) after relaxing the unity coefficient on actual inflation.

Note that using (7.8) in (7.7) we get

$$
l_{t}=\left(\phi_{f p}+\phi_{l}\right)+\phi_{3} \pi_{t}+\left(\varepsilon_{i, t}+\varepsilon_{l, t}\right) \text {, }
$$

which is the long-run Fisher parity relation. 
Stock market The third arbitrage relation links the stock market and output

$$
f_{t}=\phi_{f}+\phi_{4} y_{t}+\varepsilon_{f, t}
$$

where $f_{t}$ is the $\log$ real stock market index and $\varepsilon_{f, t}$ is a stationary disturbance

$$
\varepsilon_{f, t}=\rho_{5} \varepsilon_{f, t-1}+v_{f, t} \quad \rho_{5}<1,
$$

and $v_{f, t}$ is white noise.

From the present-value model

$$
F_{t}=E_{t}\left[\sum_{j=1}^{\infty}(1+\vartheta)^{-j} D_{t+j}\right],
$$

where $F_{t}$ is the real stock market index, $\vartheta$ is the (real, risk adjusted) discount rate and $D_{t}$ is the real dividend paid at time $t$. Assuming a constant rate of growth for dividends $(g)$, one obtains the Gordon (1962) growth model

$$
F_{t}=\frac{1+g}{\vartheta-g} D_{t}
$$

If dividends are constant the formula simplifies to

$$
\frac{D_{t}}{F_{t}}=\vartheta
$$

where the dividend yield $\left(\frac{D_{t}}{F_{t}}\right)$ equals the real risk-adjusted rate of return on capital.

Assuming a proportional relationship between real dividends and output

$$
\begin{gathered}
D_{t}=k Y_{t}^{\phi_{4}} \exp \left(\varepsilon_{d, t}\right) \\
\varepsilon_{d, t}=\rho_{6} \varepsilon_{d, t-1}+v_{d, t} \quad \rho_{6}<1,
\end{gathered}
$$

we obtain equation $(7.10)$

$$
f_{t}=\ln \left(\frac{1+g}{\vartheta-g}\right)+\ln k+\phi_{4} y_{t}+\varepsilon_{d, t} .
$$


Arbitrage between the bond and the stock markets Equations (7.9) and (7.10) have implications for the dynamics of the bond and the stock market. Consider that dividends are constant. In this case, in equation (7.10), $\phi_{f}=$ $(-\ln \vartheta+\ln k)$. It follows that

$$
f_{t}-\phi_{4} y_{t}=-\ln \vartheta+\ln k+\varepsilon_{d, t}
$$

given that $l_{t}-\phi_{3} \pi_{t}=\left(\rho+\varepsilon_{r p, t}+\varepsilon_{\rho, t}\right)+\left(\phi_{l}+v_{\pi^{*}, t}+\varepsilon_{l, t}\right)$ a suggested relation is $\left(-\ln \vartheta+\ln k+\varepsilon_{d, t}\right) \approx\left(\rho+\varepsilon_{r p, t}+\varepsilon_{\rho, t}\right)$.

Thus, even though $l_{t}$ and $\pi_{t}$ are tied in the long-run and also $f_{t}$ and $y_{t}$, in the short-run there must be a (close) negative relationship between deviations from the long-run Fisher relation and deviations from the stock market relation if, $v_{\pi^{*}, t}$ and $\varepsilon_{l, t}$, are not too volatile. In other words, the deviation from the (long-run) Fisher relation is related to the variation in the real rate of return on capital plus the variation in the risk premium, essentially the same factors that should explain the deviation from the stock market equilibrium relation.

Steady-state of the model To find the steady-state of the model, the initial values of the exogenous variables are set equal to zero. Given that $v_{j, t}$ $j=\theta, \beta, \pi^{*}, f, l, r p, m, i, d, s$ are i.i.d. with zero mean, constant variances and zero covariances, in the steady state $y_{t}=y_{t}^{*}, f_{t}=f_{t}^{*}, \pi_{t}=\pi_{t}^{*}, v_{i, t}=\varepsilon_{i, t}=0$. Thus, ignoring constants, the steady-state is as follows

$$
\begin{gathered}
\pi_{t}=\pi_{t}^{*}=\gamma_{2} \beta_{t}+\gamma_{3} \theta_{t}, \\
y_{t}=y_{t}^{*}=\gamma_{1} \theta_{t}, \\
r m_{t}=r m_{t}^{*}=\phi_{1} y_{t}^{*}=\phi_{1} \gamma_{1} \theta_{t}, \\
l_{t}=l_{t}^{*}=\phi_{3} \pi_{t}^{*}=\phi_{3} \gamma_{2} \beta_{t}+\phi_{3} \gamma_{3} \theta_{t}, \\
i_{t}=i_{t}^{*}=\phi_{3} \pi_{t}^{*}=\phi_{3} \gamma_{2} \beta_{t}+\phi_{3} \gamma_{3} \theta_{t}, \\
f_{t}=f_{t}^{*}=\phi_{4} y_{t}^{*}=\phi_{4} \gamma_{1} \theta_{t},
\end{gathered}
$$

or, more compactly,

$$
\left[\begin{array}{c}
y_{t} \\
f_{t} \\
r m_{t} \\
i_{t} \\
l_{t} \\
\pi_{t}
\end{array}\right]=\left[\begin{array}{cc}
\gamma_{1} & 0 \\
\phi_{4} \gamma_{1} & 0 \\
\phi_{1} \gamma_{1} & 0 \\
\phi_{3} \gamma_{3} & \phi_{3} \gamma_{2} \\
\phi_{3} \gamma_{3} & \phi_{3} \gamma_{2} \\
\gamma_{3} & \gamma_{2}
\end{array}\right]\left[\begin{array}{c}
\theta_{t} \\
\beta_{t}
\end{array}\right]
$$


The steady-state displays monetary neutrality as the nominal trend $\left(\beta_{t}\right)$ does not have an impact on real variables $\left(y_{t}, f_{t}, r m_{t}\right)$ in the long-run.

\subsection{Short-run predictions of the model}

To investigate the short-run behaviour of the endogenous variables included in the system it is necessary to specify an additional equation for the determination of output in the short-run.

IS equation We assume that the goods market equilibrium, in deviations from the steady state, is described by the following equation

$$
\begin{gathered}
y_{t}-y_{t}^{*}=-\alpha_{2}\left[\left(l_{t}-l_{t}^{*}\right)-\left(\pi_{t}-\pi_{t}^{*}\right)\right]+\alpha_{3}\left(f_{t}-f_{t}^{*}\right)+\varepsilon_{A D, t}, \\
\varepsilon_{A D, t}=\rho_{7} \varepsilon_{A D, t-1}+v_{A D, t} \quad \rho_{7}<1,
\end{gathered}
$$

This is an augmented IS equation where output is negatively related to the longterm real interest rate (investment component), positively related to the real stock market price index (Tobin's q / balance sheets component), and depends on a transitory shock, possibly related to fiscal policy.

Aggregate demand The joint equilibrium in financial and goods markets determines aggregate demand $(\mathrm{AD})$. Assuming that the short-term interest rate is the policy variable, the short-term Fisher parity equation can be considerd as the rule for setting the short-term rate by the monetary authority. To derive the aggregate demand function we use the term structure relation, the short-term Fisher parity and the stock market equilibrium condition in equation (2.11). The aggregate demand in deviation from the steady state, is

$$
y_{t}-y_{t}^{*}=-\phi_{2}\left(\pi_{t}-\pi_{t}^{*}\right)-\phi_{5} \varepsilon_{i, t}+\phi_{7} \varepsilon_{A D, t}+\phi_{6} \varepsilon_{f, t}-\phi_{5} \varepsilon_{l, t},
$$

where $\phi_{7}=\frac{1}{1-\alpha_{3} \phi_{4}},-\phi_{2}=-\alpha_{2}\left(\phi_{3}-1\right) \phi_{7}, \phi_{5}=\alpha_{2} \phi_{7}, \phi_{6}=\alpha_{3} \phi_{7}$. If $\phi_{7}>0$ $\left(\alpha_{3} \phi_{4}<1\right)$, then $\phi_{5}$ and $\phi_{6}$ are positive. In addition, if $\phi_{3}>1$, then also $\phi_{2}$ is positive. We assume that these (stability) conditions are met. The economic interpretation of these assumptions is that the aggregate demand curve is negatively sloped in the $\left(y_{t}, \pi_{t}\right)$ space. 
Short-term macroeconomic equilibrium The AD/AS model in deviations from the steady state can be written as follows

$$
\begin{gathered}
y_{t}-y_{t}^{*}=-\phi_{2}\left(\pi_{t}-\pi_{t}^{*}\right)-\phi_{5} \varepsilon_{i, t}+\phi_{7} \varepsilon_{A D, t}+\phi_{6} \varepsilon_{f, t}-\phi_{5} \varepsilon_{l, t}, \\
\pi_{t}-\pi_{t}^{*}=\alpha_{1}\left(y_{t}-y_{t}^{*}\right),
\end{gathered}
$$

and solved for the output and inflation gaps. The reduced form of the model is therefore

$$
\left[\begin{array}{c}
y_{t}-y_{t}^{*} \\
\pi_{t}-\pi_{t}^{*}
\end{array}\right]=\frac{1}{-\lambda}\left[\begin{array}{cccc}
\phi_{5} & -\phi_{7} & -\phi_{6} & \phi_{5} \\
\alpha_{1} \phi_{5} & -\alpha_{1} \phi_{7} & -\alpha_{1} \phi_{6} & \alpha_{1} \phi_{5}
\end{array}\right]\left[\begin{array}{c}
\varepsilon_{i, t} \\
\varepsilon_{A D, t} \\
\varepsilon_{f, t} \\
\varepsilon_{l, t}
\end{array}\right],
$$

where $-\lambda=-1-\alpha_{1} \phi_{2}$. Assuming $\phi_{3}>1$ and $\alpha_{3} \phi_{4}<1, \lambda$ is positive $(\lambda=$ $\left.1+\frac{\alpha_{1} \alpha_{2}\left(\phi_{3}-1\right)}{1-\alpha_{3} \phi_{4}}>0\right)$.

The model predicts that short- and long-term interest rate innovations have a negative impact on inflation and output, while demand and financial shocks have positive impacts on both variables. In addition, if $\alpha_{1}<1$ the impact on the inflation cycle will be smaller than the impact on the output cycle. Finally, given the recursive structure of the economy, the reduced form of the other short-term equations is given by

$$
\begin{aligned}
& {\left[\begin{array}{c}
f_{t}-f_{t}^{*} \\
r m_{t}-r m_{t}^{*} \\
i_{t}-i_{t}^{*} \\
l_{t}-l_{t}^{*}
\end{array}\right]=} \\
& =\frac{1}{-\lambda}\left[\begin{array}{ccccc}
\phi_{4} \phi_{5} & -\phi_{4} \phi_{7} & \phi_{4} \phi_{6}-\lambda & \phi_{4} \phi_{5} & 0 \\
\phi_{1} \phi_{5} & -\phi_{1} \phi_{7} & -\phi_{1} \phi_{6} & \phi_{1} \phi_{5} & -1 \\
\phi_{3} \alpha_{1} \phi_{5}-\lambda & -\phi_{3} \alpha_{1} \phi_{7} & -\phi_{3} \alpha_{1} \phi_{6} & \phi_{3} \alpha_{1} \phi_{5} & 0 \\
\phi_{3} \alpha_{1} \phi_{5}-\lambda & -\phi_{3} \alpha_{1} \phi_{7} & -\phi_{3} \alpha_{1} \phi_{6} & \phi_{3} \alpha_{1} \phi_{5}-\lambda & 0
\end{array}\right]\left[\begin{array}{c}
\varepsilon_{i, t} \\
\varepsilon_{A D, t} \\
\varepsilon_{f, t} \\
\varepsilon_{l, t} \\
\varepsilon_{m, t}
\end{array}\right] \text {, }
\end{aligned}
$$

The model predicts that a short-term interest rate innovation has a negative impact on the stock market cycle and the real money balances gap, and a positive impact on the short- and long-term interest rate cycles if $\lambda>\phi_{3} \alpha_{1} \phi_{5}$ (i.e. if $\left.\alpha_{3} \phi_{4}+\alpha_{1} \alpha_{2}<1\right)$. On the other hand, a long-term rate innovation will have a negative impact on the stock market, real money balances and short term rate 
cycles, and a positive impact on the long-term interest rate cycle. Finally, the aggregate demand and financial shocks have a positive impact on all the variables. A money demand shock can be added (the stationary linear combination of spreads) affecting only the real balances gap. Note that the latter shock is not structural in our framework, since it can be related to the short- and long-term interest rate shocks, and to financial innovations. 
Table 1

Cointegration analysis

Cointegration tests

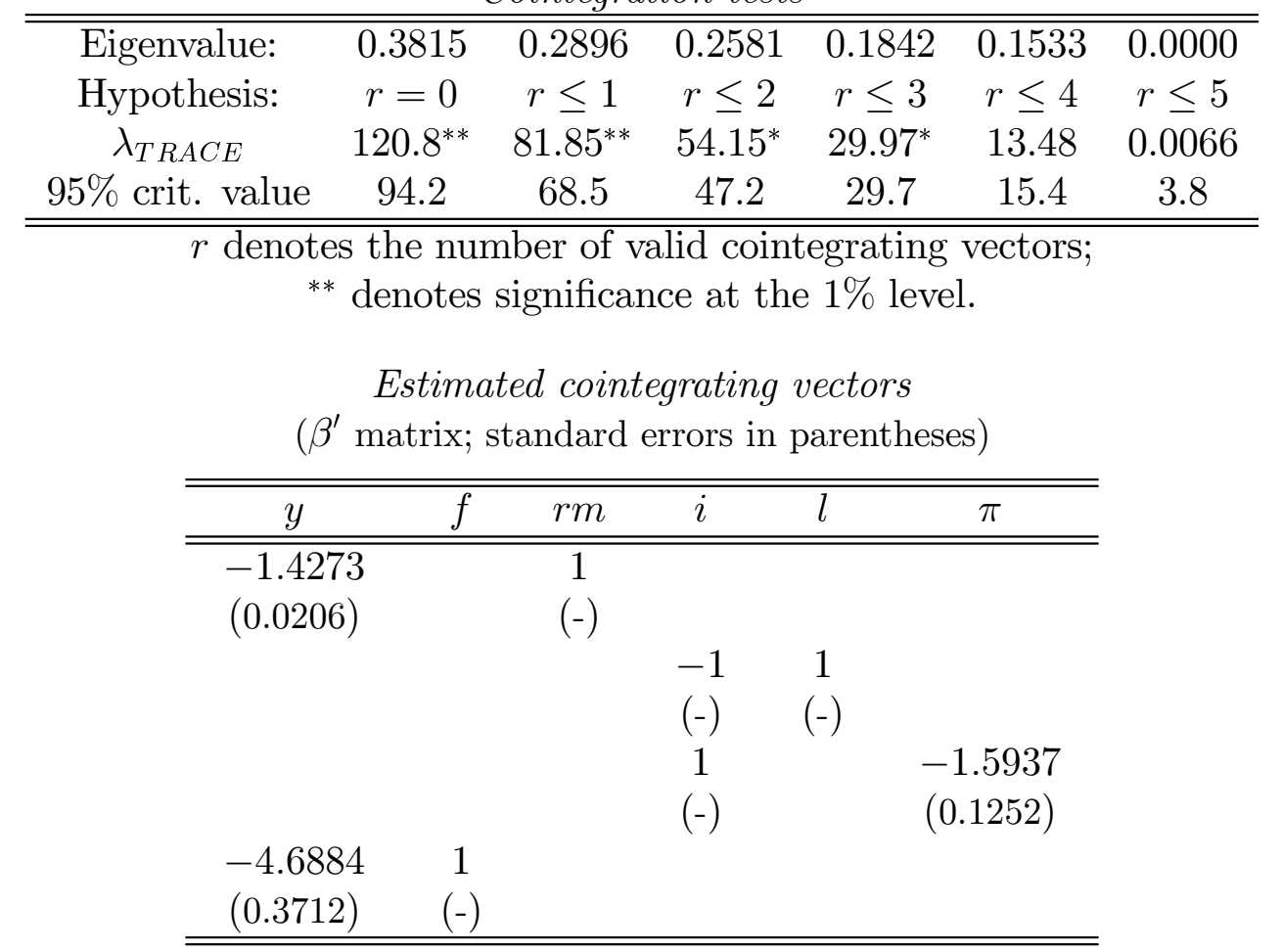


Estimated factor loadings matrix ( $\alpha$ matrix; standard errors in parentheses)

\begin{tabular}{|c|c|c|c|c|}
\hline$\Delta y$ & - & $\begin{array}{l}0.4086 \\
(0.2151)\end{array}$ & - & $\begin{array}{l}0.0080 \\
(0.0026)\end{array}$ \\
\hline$\Delta f$ & - & - & - & - \\
\hline$\Delta r m$ & $\begin{array}{c}-0.1039 \\
(0.0219)\end{array}$ & - & - & $\begin{array}{l}0.0055 \\
(0.0016)\end{array}$ \\
\hline$\Delta i$ & $\begin{array}{l}-0.0327 \\
(0.0075)\end{array}$ & $\begin{array}{l}0.1406 \\
(0.0527)\end{array}$ & - & - \\
\hline$\Delta l$ & 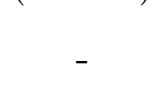 & $\begin{array}{l}-0.074 \\
(0.0408)\end{array}$ & $\begin{array}{c}-0.0628 \\
(0.0305)\end{array}$ & - \\
\hline$\Delta \pi$ & - & $\begin{array}{l}0.1880 \\
(0.0872)\end{array}$ & $\begin{array}{l}0.3039 \\
(0.0739)\end{array}$ & - \\
\hline
\end{tabular}


Table 2

The short-run model

( standard errors in parentheses)

\begin{tabular}{ccccccc}
\hline \hline & $\Delta y_{t}$ & $\Delta f_{t}$ & $\Delta r m_{t}$ & $\Delta i_{t}$ & $\Delta l_{t}$ & $\Delta \pi_{t}$ \\
\hline \hline \multirow{2}{*}{$\Delta y_{t-1}$} & 0.0408 & 2.8221 & -0.1117 & -0.0342 & 0.0166 & -0.0276 \\
& $(0.1151)$ & $(1.6135)$ & $(0.0901)$ & $(0.0290)$ & $(0.0225)$ & $(0.0526)$ \\
$\Delta f_{t-1}$ & -0.0112 & 0.0669 & -0.0091 & 0.0042 & 0.0018 & 0.0016 \\
& $(0.0083)$ & $(0.1162)$ & $(0.0065)$ & $(0.0021)$ & $(0.0016)$ & $(0.0038)$ \\
$\Delta r m_{t-1}$ & 0.1248 & 3.1560 & 0.5132 & 0.0818 & 0.0277 & -0.0162 \\
& $(0.1423)$ & $(1.9947)$ & $(0.1114)$ & $(0.0359)$ & $(0.0278)$ & $(0.0651)$ \\
$\Delta i_{t-1}$ & 0.6458 & 11.405 & -0.2391 & 0.2645 & -0.0298 & 0.4583 \\
& $(0.5024)$ & $(7.0395)$ & $(0.3933)$ & $(0.1267)$ & $(0.0981)$ & $(0.2297)$ \\
$\Delta l_{t-1}$ & 0.3600 & -29.962 & -0.8989 & 0.3858 & 0.6431 & 0.1223 \\
& $(0.5977)$ & $(8.3754)$ & $(0.4679)$ & $(0.1507)$ & $(0.1167)$ & $(0.2733)$ \\
$\Delta \pi_{t-1}$ & 0.1181 & -1.0056 & -0.1520 & 0.0134 & -0.0303 & -0.2832 \\
& $(0.2511)$ & $(3.5181)$ & $(0.1965)$ & $(0.0633)$ & $(0.0490)$ & $(0.1148)$ \\
$E R B_{t-1}$ & 0.0744 & 1.1811 & -0.0948 & -0.0257 & 0.0042 & 0.0169 \\
& $(0.0383)$ & $(0.5372)$ & $(0.0300)$ & $(0.0097)$ & $(0.0075)$ & $(0.0175)$ \\
$T S_{t-1}$ & 0.2866 & 0.5537 & -0.0919 & 0.0999 & -0.1172 & 0.1948 \\
& $(0.2498)$ & $(3.5003)$ & $(0.1955)$ & $(0.0630)$ & $(0.0488)$ & $(0.1142)$ \\
$F H_{t-1}$ & -0.1705 & -6.9754 & -0.1905 & -0.0604 & -0.0938 & 0.2916 \\
& $(0.2159)$ & $(3.0253)$ & $(0.1690)$ & $(0.0544)$ & $(0.0422)$ & $(0.0987)$ \\
SM $M_{t-1}$ & 0.0069 & -0.0837 & 0.0048 & -0.0001 & 0.0002 & -0.0001 \\
& $(0.0027)$ & $(0.0385)$ & $(0.0022)$ & $(0.0007)$ & $(0.0005)$ & $(0.0013)$ \\
constant & 0.9848 & 2.7198 & -0.3403 & -0.1858 & 0.0403 & 0.1115 \\
& $(0.3105)$ & $(4.3505)$ & $(0.2430)$ & $(0.0783)$ & $(0.0606)$ & $(0.1420)$ \\
\hline \hline ERB: exc
\end{tabular}

(ERB: excess real balance; TS: term structure; FH: Fisher hypothesis; SM: Gordon growth model) 
Table 3

Specification tests

( $\mathrm{P}$-values in parentheses)

\begin{tabular}{ccccc}
\hline \hline & AR 1-5 & Normality & ARCH 4 & White \\
\hline \hline \multirow{2}{*}{$\Delta y_{t}$} & 1.4347 & 8.8522 & 0.9325 & 0.2419 \\
& $(0.2237)$ & $(0.0120)$ & $(0.4511)$ & $(0.9952)$ \\
$\Delta f_{t}$ & 2.2056 & 4.4072 & 0.3358 & 0.3339 \\
& $(0.0643)$ & $(0.1104)$ & $(0.8528)$ & $(0.9752)$ \\
$\Delta r m_{t}$ & 1.3745 & 0.0465 & 2.1545 & 1.8691 \\
& $(0.2454)$ & $(0.9770)$ & $(0.0846)$ & $(0.2895)$ \\
$\Delta i_{t}$ & 0.5845 & 6.4197 & 2.2245 & 1.1801 \\
& $(0.7117)$ & $(0.0404)$ & $(0.0765)$ & $(0.4996)$ \\
$\Delta l_{t}$ & 1.5837 & 3.4599 & 0.4509 & 1.02 \\
& $(0.1771)$ & $(0.1773)$ & $(0.7714)$ & $(0.5755)$ \\
$\Delta \pi_{t}$ & 0.5230 & 3.2669 & 0.0502 & 0.2487 \\
& $(0.7580)$ & $(0.1953)$ & $(0.9951)$ & $(0.9944)$ \\
\hline \hline
\end{tabular}

AR 1-5: F(5,65); Normality: $\chi_{(2)}^{2}$; ARCH 4: F(4,62); White: F(65,4). 
Table 4

The estimated common trends model

Panel A: Long-run effects of permanent shocks (\%) (matrix $\Gamma_{g}$ ) (simulated standard errors in parentheses, annualised values)

\begin{tabular}{c|cc}
\hline \hline Variable & \multicolumn{2}{|c}{ Shock } \\
& $\tau_{\theta}$ & $\tau_{\beta}$ \\
\hline \hline \multirow{2}{*}{$y$} & 0.545 & - \\
& $(0.260)$ & \\
$f$ & 2.553 & - \\
& $(1.217)$ & \\
$r m$ & 0.777 & - \\
& $(0.370)$ & \\
$i$ & -0.066 & 0.585 \\
& $(0.363)$ & $(0.146)$ \\
$l$ & -0.066 & 0.585 \\
& $(0.363)$ & $(0.146)$ \\
$\pi$ & -0.041 & 0.367 \\
& $(0.228)$ & $(0.091)$ \\
\hline \hline
\end{tabular}

Panel B: Contemporaneous impact of structural shocks (\%) (matrix $\left.\Gamma_{0}\right)$

(simulated standard errors in parentheses, annualised values)

\begin{tabular}{|c|c|c|c|c|c|c|}
\hline \multirow[t]{2}{*}{ Variable } & \multirow[b]{2}{*}{$\tau_{\theta}$} & \multirow[b]{2}{*}{$\tau_{\beta}$} & \multicolumn{3}{|c|}{ Shock } & \multirow[b]{2}{*}{$v_{F H}$} \\
\hline & & & $v_{T S}$ & $v_{S M}$ & $v_{A D}$ & \\
\hline$y$ & $\begin{array}{l}-0.097 \\
(0.105)\end{array}$ & $\begin{array}{l}-0.090 \\
(0.096)\end{array}$ & $\begin{array}{l}0.0 \\
(-)\end{array}$ & $\begin{array}{l}0.0 \\
(-)\end{array}$ & $\begin{array}{l}0.395 \\
(0.041)\end{array}$ & $\begin{array}{l}0.089 \\
(0.056)\end{array}$ \\
\hline$f$ & $\begin{array}{l}2.912 \\
(1.137)\end{array}$ & $\begin{array}{l}2.309 \\
(1.370)\end{array}$ & $\begin{array}{l}-1.117 \\
(1.546)\end{array}$ & $\begin{array}{l}-3.862 \\
(1.002)\end{array}$ & $\begin{array}{l}1.879 \\
(1.202)\end{array}$ & $\begin{array}{l}0.507 \\
(0.93)\end{array}$ \\
\hline $\mathrm{rm}$ & $\begin{array}{l}0.102 \\
(0.081)\end{array}$ & $\begin{array}{l}0.082 \\
(0.085)\end{array}$ & $\begin{array}{l}0.0 \\
(-)\end{array}$ & $\begin{array}{l}0.160 \\
(0.041)\end{array}$ & $\begin{array}{l}0.117 \\
(0.052)\end{array}$ & $\begin{array}{l}-0.234 \\
(0.042)\end{array}$ \\
\hline$i$ & $\begin{array}{c}-0.277 \\
(0.111)\end{array}$ & $\begin{array}{l}0.179 \\
(0.086)\end{array}$ & $\begin{array}{l}-0.218 \\
(0.194)\end{array}$ & $\begin{array}{l}0.009 \\
(0.077)\end{array}$ & $\begin{array}{l}0.074 \\
(0.075)\end{array}$ & $\begin{array}{l}-0.142 \\
(0.058)\end{array}$ \\
\hline$l$ & $\begin{array}{l}-0.185 \\
(0.086)\end{array}$ & $\begin{array}{l}0.161 \\
(0.071)\end{array}$ & $\begin{array}{l}0.127 \\
(0.055)\end{array}$ & $\begin{array}{l}0.001 \\
(0.063)\end{array}$ & $\begin{array}{l}0.068 \\
(0.069)\end{array}$ & $\begin{array}{l}-0.157 \\
(0.047)\end{array}$ \\
\hline$\pi$ & $\begin{array}{l}-0.117 \\
(0.152)\end{array}$ & $\begin{array}{l}0.365 \\
(0.156)\end{array}$ & $\begin{array}{l}0.0 \\
(-)\end{array}$ & $\begin{array}{l}0.0 \\
(-)\end{array}$ & $\begin{array}{l}0.0 \\
(-)\end{array}$ & $\begin{array}{l}0.645 \\
(0.071)\end{array}$ \\
\hline
\end{tabular}


Panel C: Contemporaneous impact of structural shocks (\%) (matrix $\Lambda$ ) (simulated standard errors in parentheses)

\begin{tabular}{c|l|l|llll}
\hline \hline Variable & & & \multicolumn{3}{|c}{ Shock } & \\
& $\tau_{\theta}$ & $\tau_{\beta}$ & $v_{T S}$ & $v_{S M}$ & $v_{A D}$ & $v_{F H}$ \\
\hline \hline \multirow{2}{*}{$E R B$} & 0.241 & 0.209 & 0.0 & 0.160 & -0.447 & -0.361 \\
& $(0.153)$ & $(0.157)$ & $(-)$ & $(0.041)$ & $(0.069)$ & $(0.086)$ \\
\multirow{2}{*}{$T S H$} & 0.023 & -0.004 & 0.086 & -0.002 & -0.001 & -0.004 \\
& $(0.021)$ & $(0.024)$ & $(0.052)$ & $(0.018)$ & $(0.014)$ & $(0.013)$ \\
$F H$ & -0.022 & -0.101 & -0.054 & 0.002 & 0.018 & -0.293 \\
& $(0.064)$ & $(0.071)$ & $(0.049)$ & $(0.020)$ & $(0.020)$ & $(0.031)$ \\
$S M H$ & 3.368 & 2.729 & -1.116 & -3.862 & 0.026 & 0.090 \\
& $(1.077)$ & $(1.427)$ & $(1.571)$ & $(0.985)$ & $(1.165)$ & $(0.969)$ \\
\hline \hline
\end{tabular}


Table 5

The estimated common trends model

Panel A: Forecast error variance decomposition (short term)

\begin{tabular}{c|cccccccccccc}
\hline \hline & \multicolumn{1}{|c}{1 quarter } & \multicolumn{1}{c}{1 year } \\
& $\tau_{\theta}$ & $\tau_{\beta}$ & $v_{T S}$ & $v_{S M}$ & $v_{A D}$ & $v_{F H}$ & $\tau_{\theta}$ & $\tau_{\beta}$ & $v_{T S}$ & $v_{S M}$ & $v_{A D}$ & $v_{F H}$ \\
\hline \hline$y$ & 0.05 & 0.05 & 0.00 & 0.00 & 0.86 & 0.04 & 0.05 & 0.04 & 0.00 & 0.01 & 0.88 & 0.02 \\
$f$ & 0.25 & 0.16 & 0.04 & 0.44 & 0.10 & 0.01 & 0.46 & 0.12 & 0.11 & 0.17 & 0.06 & 0.08 \\
$r m$ & 0.10 & 0.06 & 0.00 & 0.23 & 0.12 & 0.49 & 0.28 & 0.02 & 0.00 & 0.28 & 0.14 & 0.28 \\
$i$ & 0.42 & 0.18 & 0.26 & 0.00 & 0.03 & 0.11 & 0.32 & 0.37 & 0.09 & 0.00 & 0.15 & 0.07 \\
$l$ & 0.33 & 0.25 & 0.15 & 0.00 & 0.04 & 0.23 & 0.29 & 0.47 & 0.07 & 0.00 & 0.07 & 0.10 \\
$\pi$ & 0.02 & 0.24 & 0.00 & 0.00 & 0.00 & 0.74 & 0.13 & 0.47 & 0.01 & 0.00 & 0.01 & 0.38 \\
\hline \hline
\end{tabular}

Panel B: Forecast error variance decomposition (medium term)

\begin{tabular}{c|cccccccccccc}
\hline \hline & \multicolumn{1}{|c}{3 year } & \multicolumn{1}{c}{5 year } \\
& $\tau_{\theta}$ & $\tau_{\beta}$ & $v_{T S}$ & $v_{S M}$ & $v_{A D}$ & $v_{F H}$ & $\tau_{\theta}$ & $\tau_{\beta}$ & $v_{T S}$ & $v_{S M}$ & $v_{A D}$ & $v_{F H}$ \\
\hline \hline$y$ & 0.34 & 0.08 & 0.01 & 0.01 & 0.53 & 0.03 & 0.69 & 0.04 & 0.00 & 0.00 & 0.25 & 0.02 \\
$f$ & 0.72 & 0.06 & 0.04 & 0.08 & 0.05 & 0.05 & 0.78 & 0.04 & 0.03 & 0.05 & 0.06 & 0.04 \\
$r m$ & 0.43 & 0.02 & 0.01 & 0.10 & 0.35 & 0.09 & 0.73 & 0.02 & 0.00 & 0.04 & 0.17 & 0.04 \\
$i$ & 0.24 & 0.60 & 0.02 & 0.00 & 0.12 & 0.02 & 0.17 & 0.73 & 0.02 & 0.00 & 0.07 & 0.01 \\
$l$ & 0.18 & 0.74 & 0.02 & 0.00 & 0.03 & 0.03 & 0.13 & 0.82 & 0.01 & 0.00 & 0.02 & 0.02 \\
$\pi$ & 0.15 & 0.65 & 0.01 & 0.00 & 0.02 & 0.17 & 0.12 & 0.75 & 0.00 & 0.00 & 0.02 & 0.11 \\
\hline \hline
\end{tabular}

Panel C: Forecast error variance decomposition (long term)

\begin{tabular}{c|ccc}
\hline \hline Variable & \multicolumn{3}{|c}{$\infty$} \\
& $\tau_{\theta}$ & $\tau_{\beta}$ & $v_{\text {joint }}$ \\
\hline \hline$y$ & 1 & 0.0 & 0.0 \\
$f$ & 1 & 0.0 & 0.0 \\
$r m$ & 1 & 0.0 & 0.0 \\
$i$ & 0.01 & 0.99 & 0.0 \\
$l$ & 0.01 & 0.99 & 0.0 \\
$\pi$ & 0.01 & 0.99 & 0.0 \\
\hline \hline
\end{tabular}


Table 6

Cyclical components: correlation matrix Panel A: Overall cycle (1980:3 - 2000:4)

\begin{tabular}{c|cccccc}
\hline \hline Variable & & & & & & \\
& $y$ & $f$ & $r m$ & $i$ & $l$ & $\pi$ \\
\hline \hline$y$ & 1 & & & & & \\
$f$ & -0.41 & 1 & & & & \\
$r m$ & 0.83 & -0.72 & 1 & & & \\
$i$ & 0.52 & -0.42 & 0.59 & 1 & & \\
$l$ & 0.12 & -0.62 & 0.27 & 0.53 & 1 & \\
$\pi$ & 0.25 & -0.30 & 0.24 & 0.12 & -0.14 & 1 \\
\hline \hline
\end{tabular}

Panel B: Dynamics towards and along the attractor (1986:1 - 2000:4)

\begin{tabular}{c|cccccc}
\hline \hline Variable & & & & & & \\
& $y$ & $f$ & $r m$ & $i$ & $l$ & $\pi$ \\
\hline \hline$y$ & - & -0.72 & 0.91 & 0.86 & 0.85 & 0.84 \\
$f$ & 0.04 & - & -0.87 & -0.87 & -0.89 & -0.90 \\
$r m$ & 0.66 & -0.55 & - & 0.87 & 0.82 & 0.90 \\
$i$ & 0.77 & -0.11 & 0.82 & - & 0.95 & 0.85 \\
$l$ & 0.56 & -0.60 & 0.75 & 0.53 & - & 0.83 \\
$\pi$ & 0.43 & -0.02 & 0.19 & 0.33 & -0.73 & - \\
\hline \hline
\end{tabular}

dynamics towards the attractor: bottom triangular matrix; dynamics along the attractor: upper triangular matrix. 
Table 7

Cyclical components (1986:1 - 2000:4): decomposition

(Proportion of total variance explained by each factor:

proportions may not sum to one due to sample effects)

\section{Panel A: Cyclical components}

\begin{tabular}{ccc}
\hline \hline Variable & & \\
& $D T A$ & $D A A$ \\
\hline \hline$y$ & 0.34 & 0.45 \\
$f$ & 0.34 & 0.33 \\
$r m$ & 0.29 & 0.41 \\
$i$ & 0.44 & 0.36 \\
$l$ & 0.28 & 0.42 \\
$\pi$ & 0.65 & 0.29 \\
\hline \hline
\end{tabular}

Panel B: Dynamics along and towards the attractor

\begin{tabular}{c|c|c|cccc}
\hline \hline Variable & & & & & & \\
& $\tau_{\theta}$ & $\tau_{\beta}$ & $v_{T S}$ & $v_{S M}$ & $v_{A D}$ & $v_{F H}$ \\
\hline \hline$y$ & 1.00 & 0.02 & 0.01 & 0.01 & 0.90 & 0.02 \\
$f$ & 0.67 & 0.12 & 0.19 & 0.32 & 0.56 & 0.16 \\
$r m$ & 0.91 & 0.01 & 0.01 & 0.15 & 0.75 & 0.12 \\
$i$ & 0.58 & 0.18 & 0.11 & 0.00 & 0.78 & 0.11 \\
$l$ & 0.53 & 0.29 & 0.21 & 0.03 & 0.53 & 0.28 \\
$\pi$ & 0.90 & 0.07 & 0.04 & 0.02 & 0.17 & 0.77 \\
\hline \hline
\end{tabular}

The columns in Panel A report the proportion of total cycle variance explained by the dynamics towards the attractor $(D T A)$ and the dynamics along the attractor $(D A A)$. The first two columns in Panel $\mathrm{B}$ report the proportion of the dynamics along the attractor variance explained by each permanent shock; the last four columns in Panel B report the proportion of the dynamics towards the attractor variance explained by each transitory shock. 

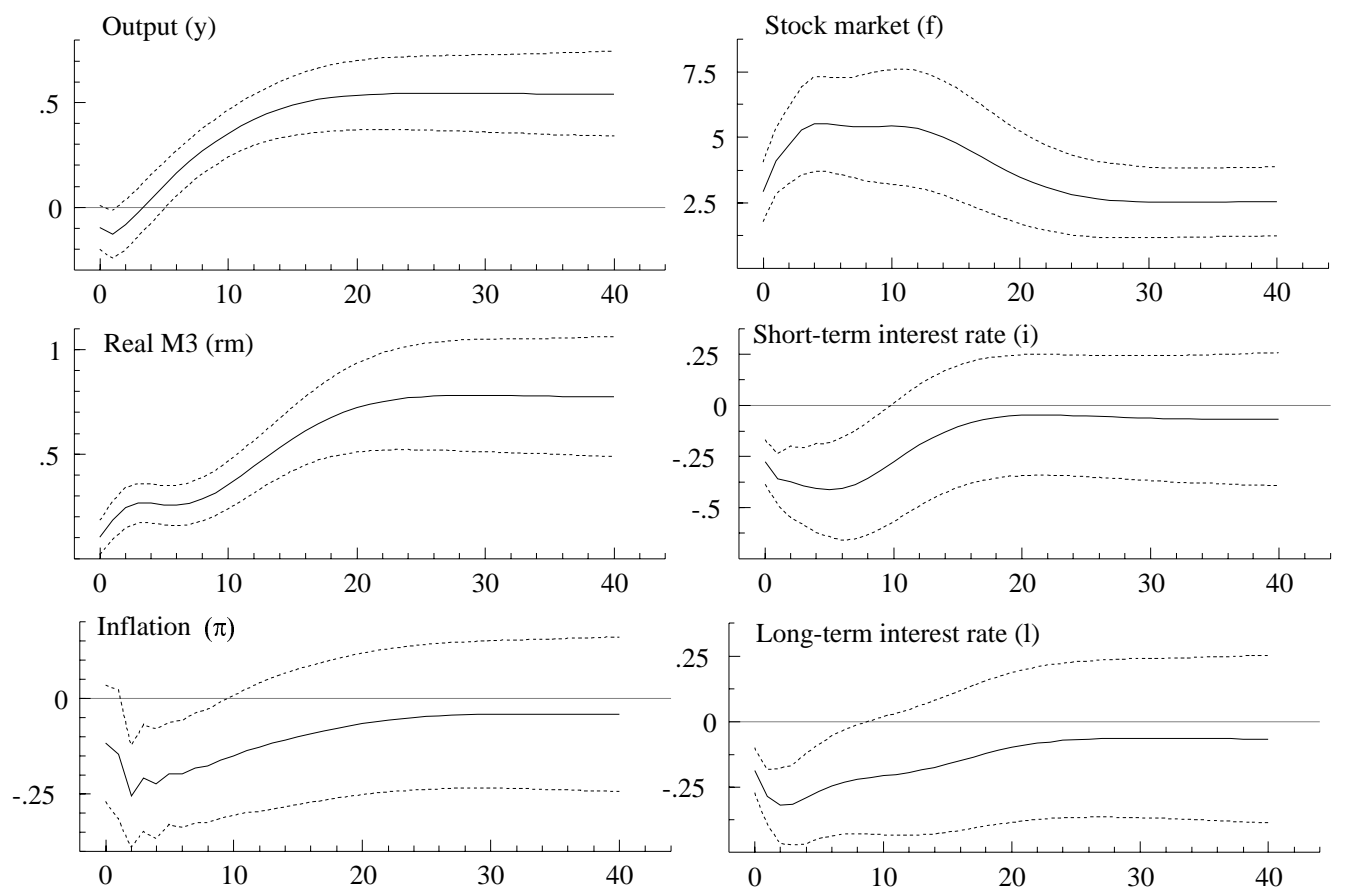

Figure 1: Impulse responses (\%) to the real permanent shock (productivity shock) 

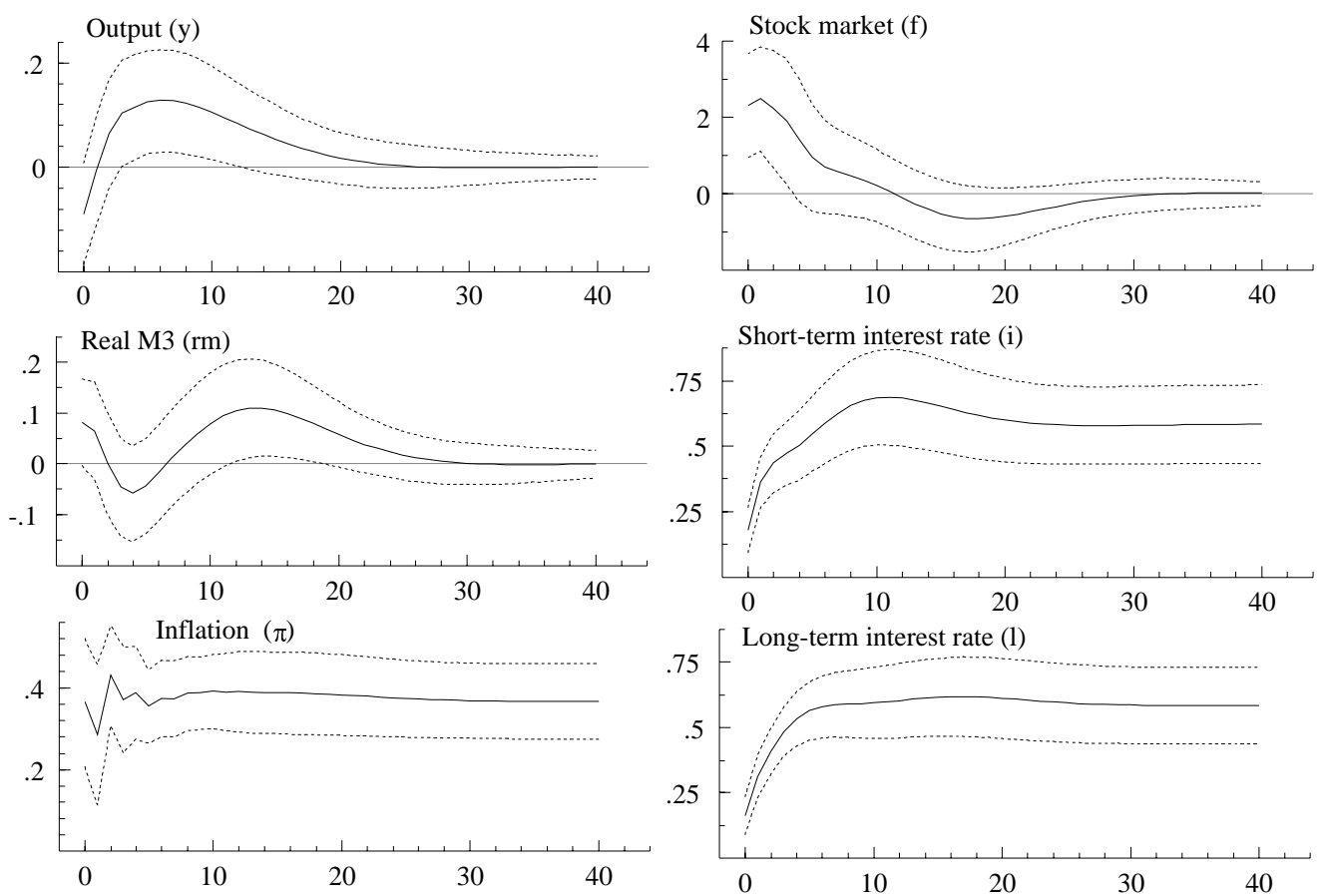

Figure 2: Impulse responses (\%) to the nominal permanent shock 

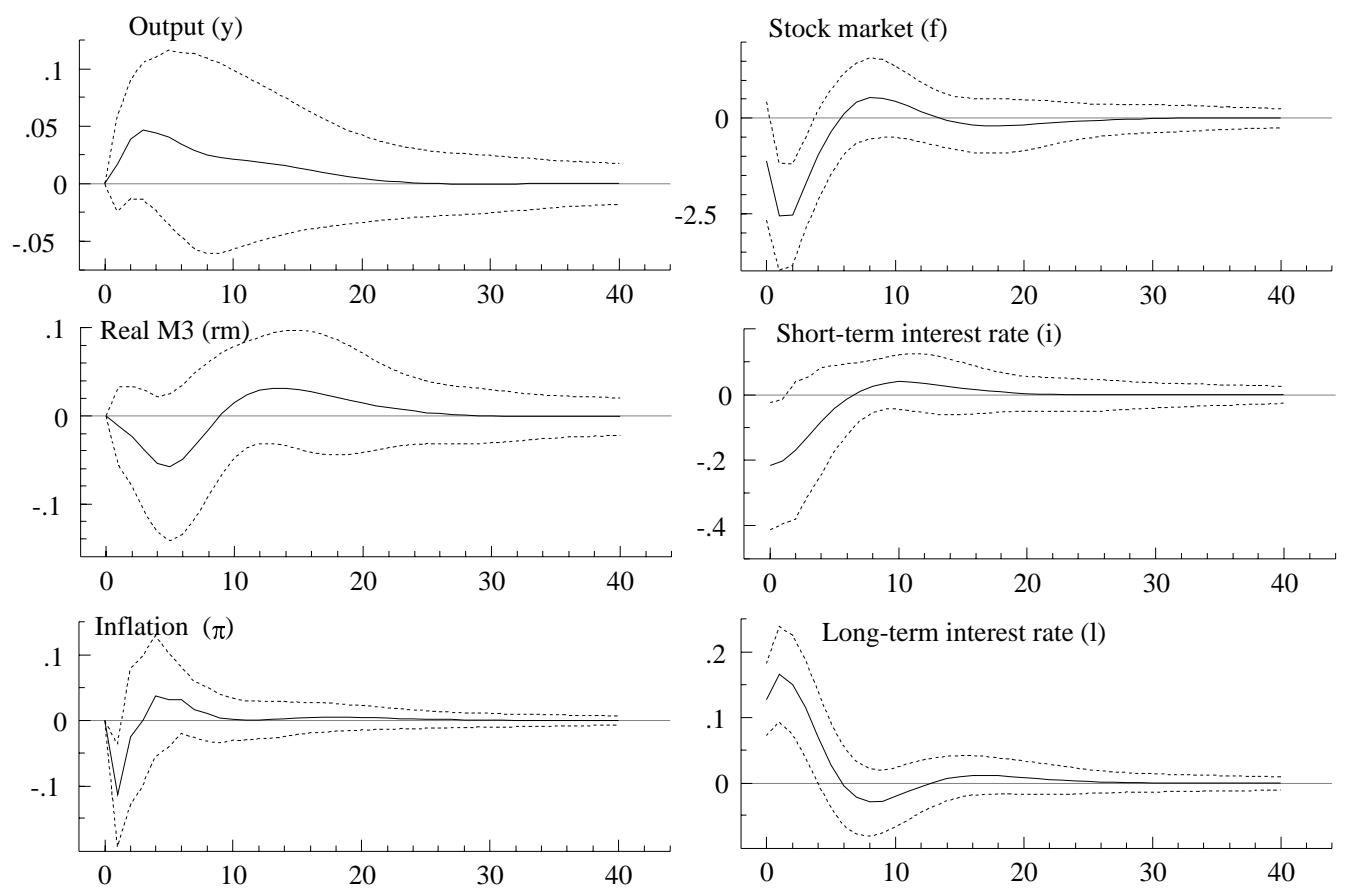

Figure 3: Impulse responses (\%) to transitory term structure shocks 

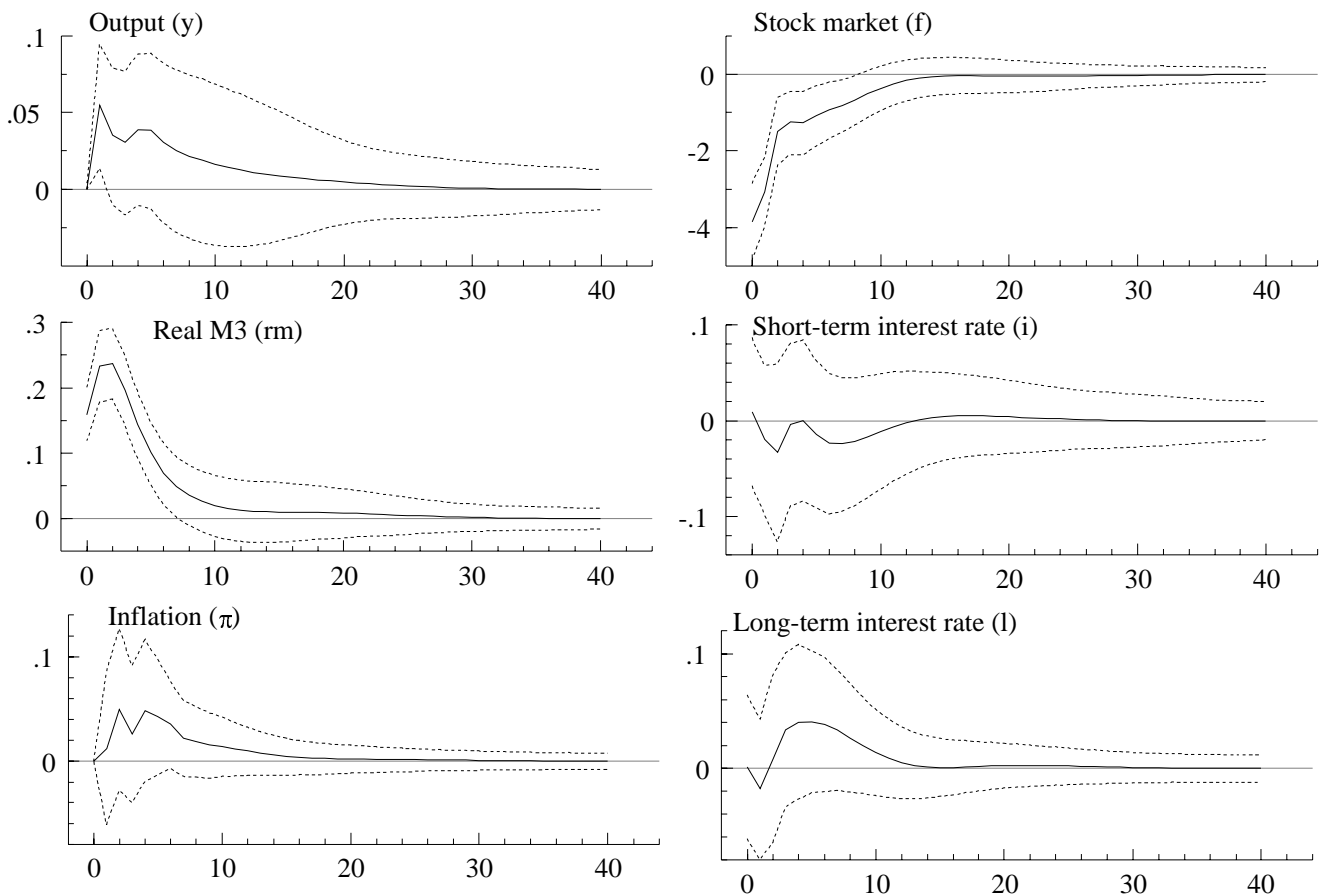

Figure 4: Impulse responses (\%) to transitory liquidity preference shocks 

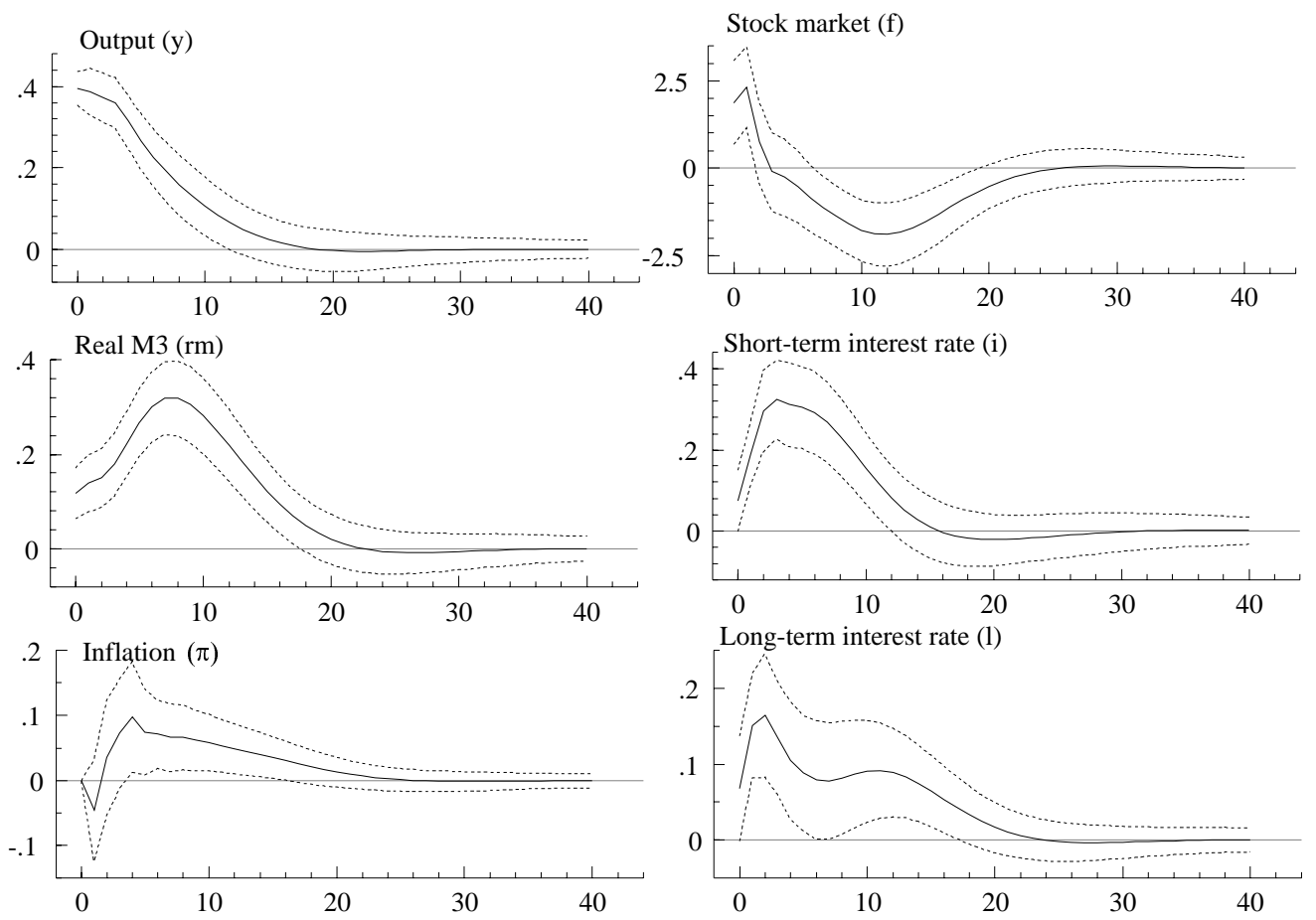

Figure 5: Impulse responses to transitory aggregate demand shocks 

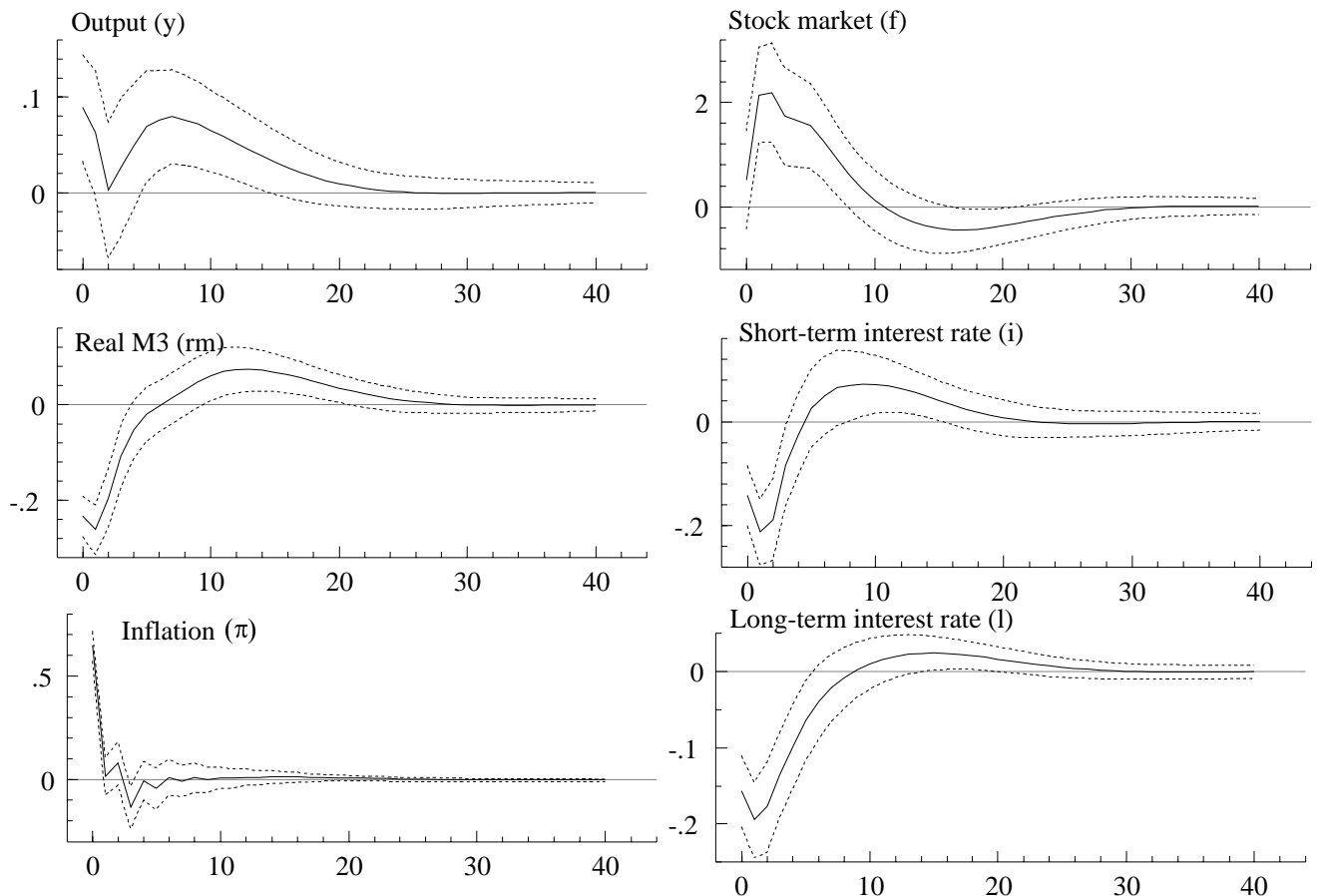

Figure 6: Impulse responses (\%) to Fisher parity equation shocks 

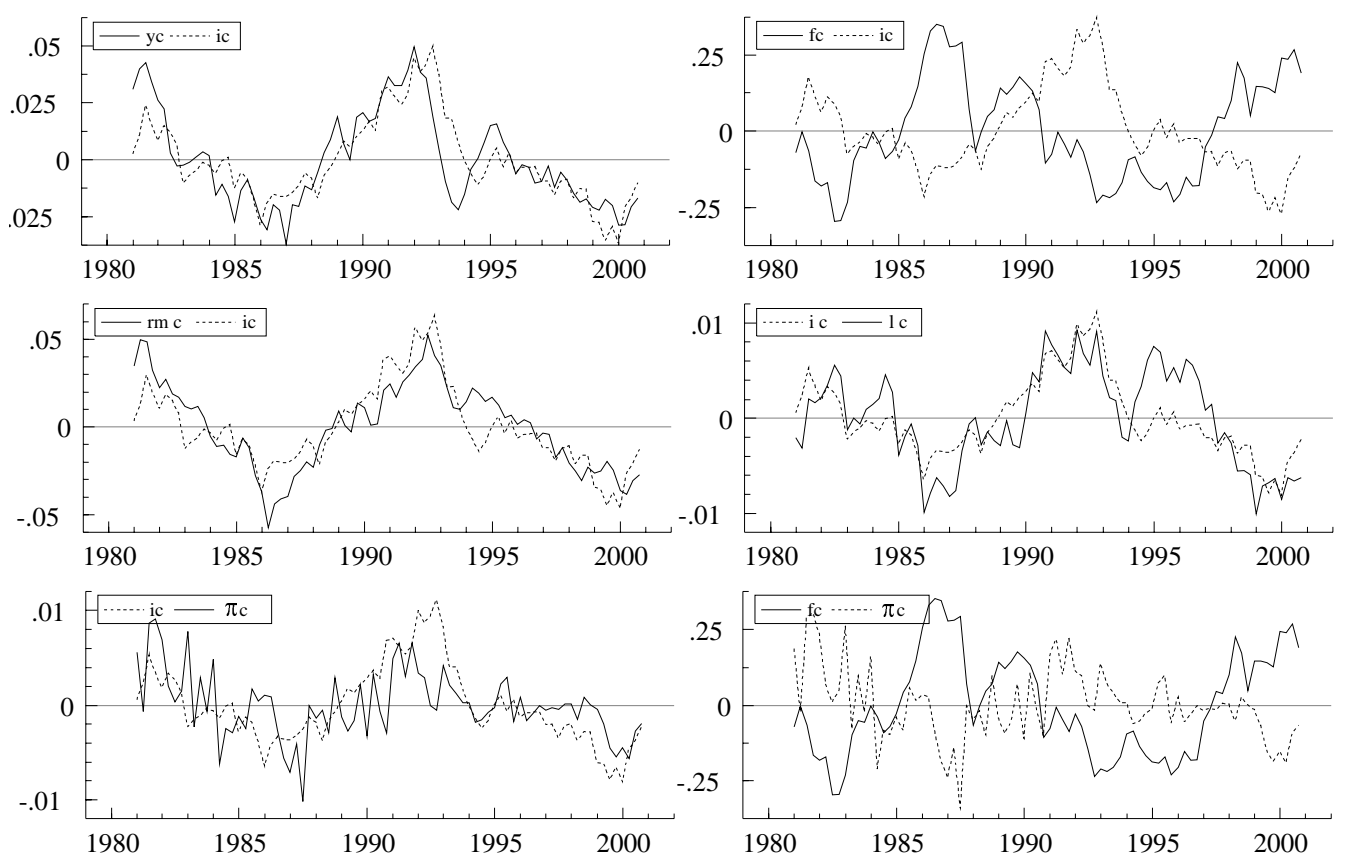

Figure 7: Cyclical components: real GDP (yc), real stock market index (fc), real money balances (rm c), short term nominal interest rate (ic), long term nominal interest rate (lc), GDP inflation $(\pi c)$. 

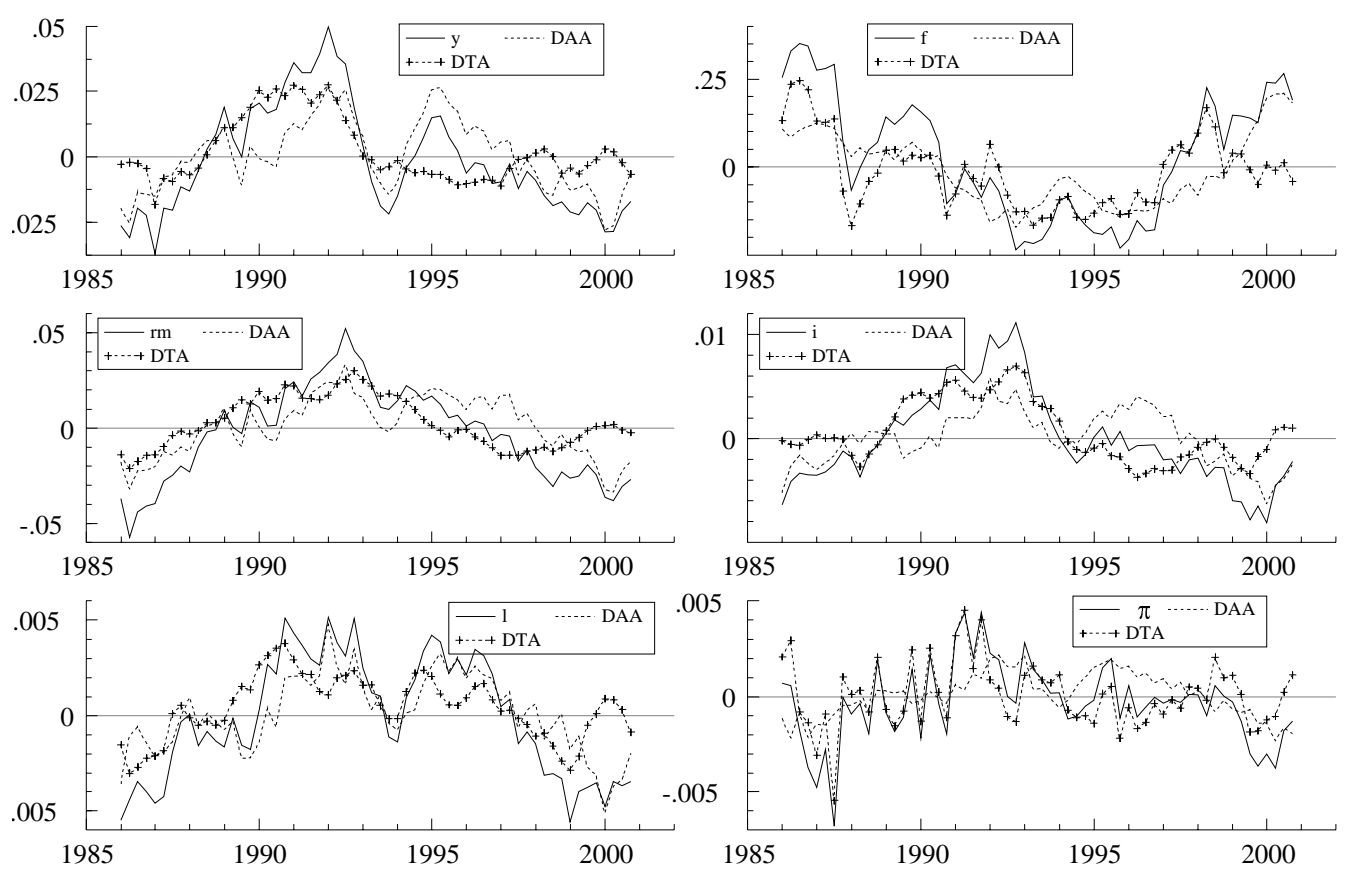

Figure 8: Decomposition of the overall cycle in dynamics towards the attractor (DTA) and dynamics along the attractor (DAA) (output (y), stock market index (f), real money balances ( $\mathrm{rm}$ ), short term interest rate (i), long term interest rate $(1)$, inflation $(\pi)$ ). 

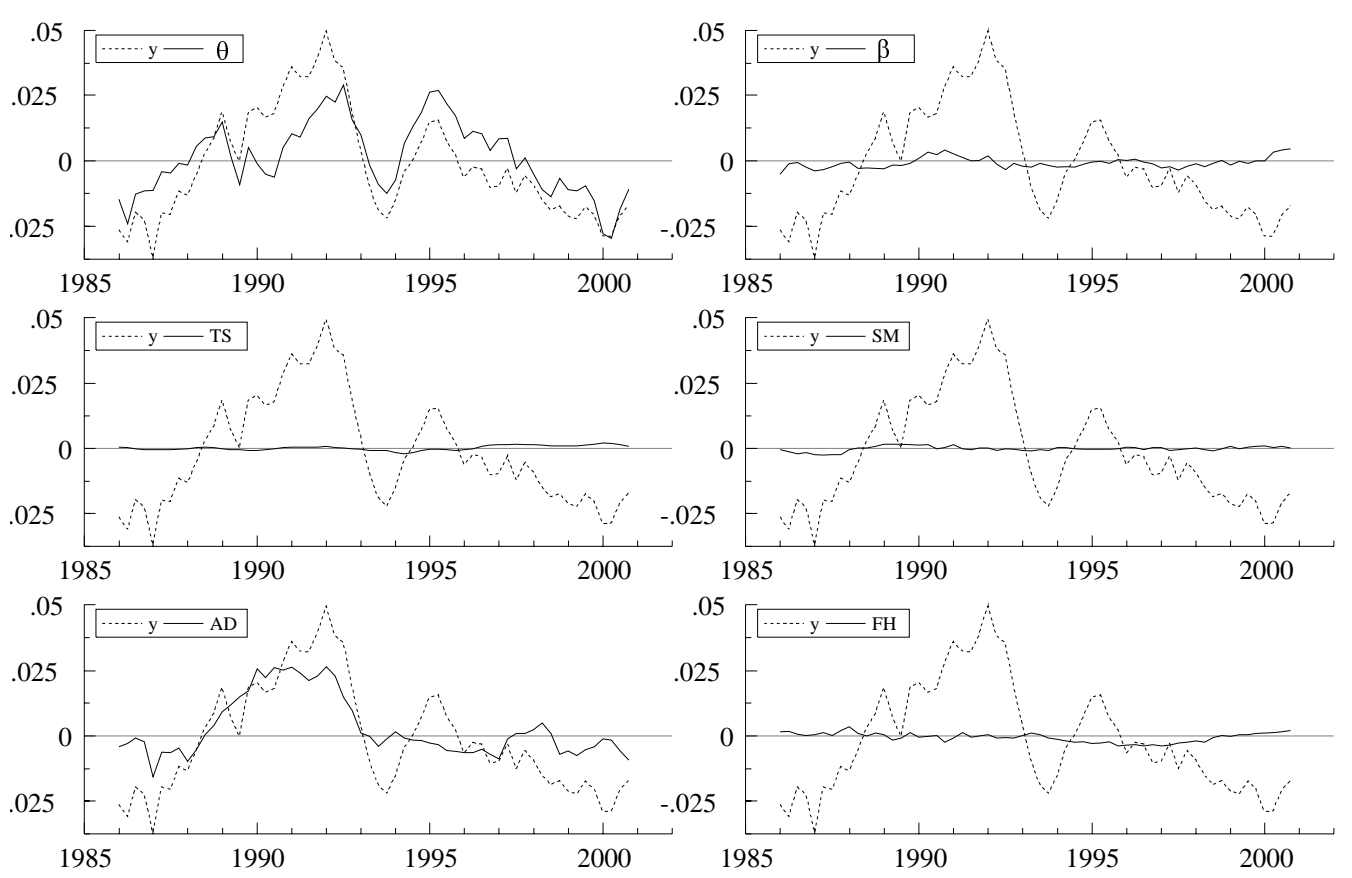

Figure 9: Historical decomposition of the output cycle $(\theta$ : real permanent shock; $\beta$ : nominal permanent shock, TS: term structure shock; SM: liquidity preference shock; AD: aggregate demand shock; FH: Fisher parity shock). 

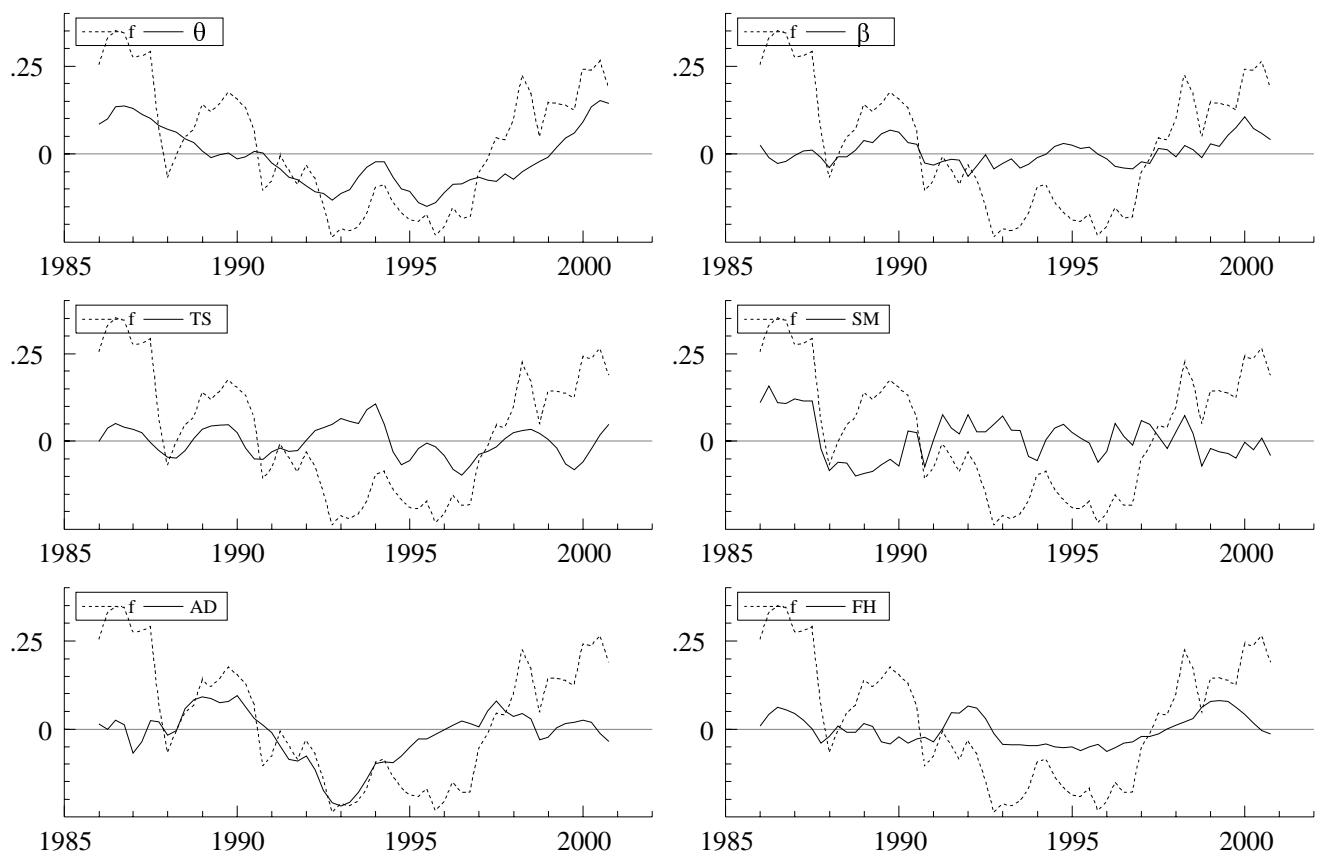

Figure 10: Historical decomposition of the stock market cycle $(\theta$ : real permanent shock; $\beta$ : nominal permanent shock, TS: term structure shock; SM: liquidity preference shock; AD: aggregate demand shock; FH: Fisher parity shock). 

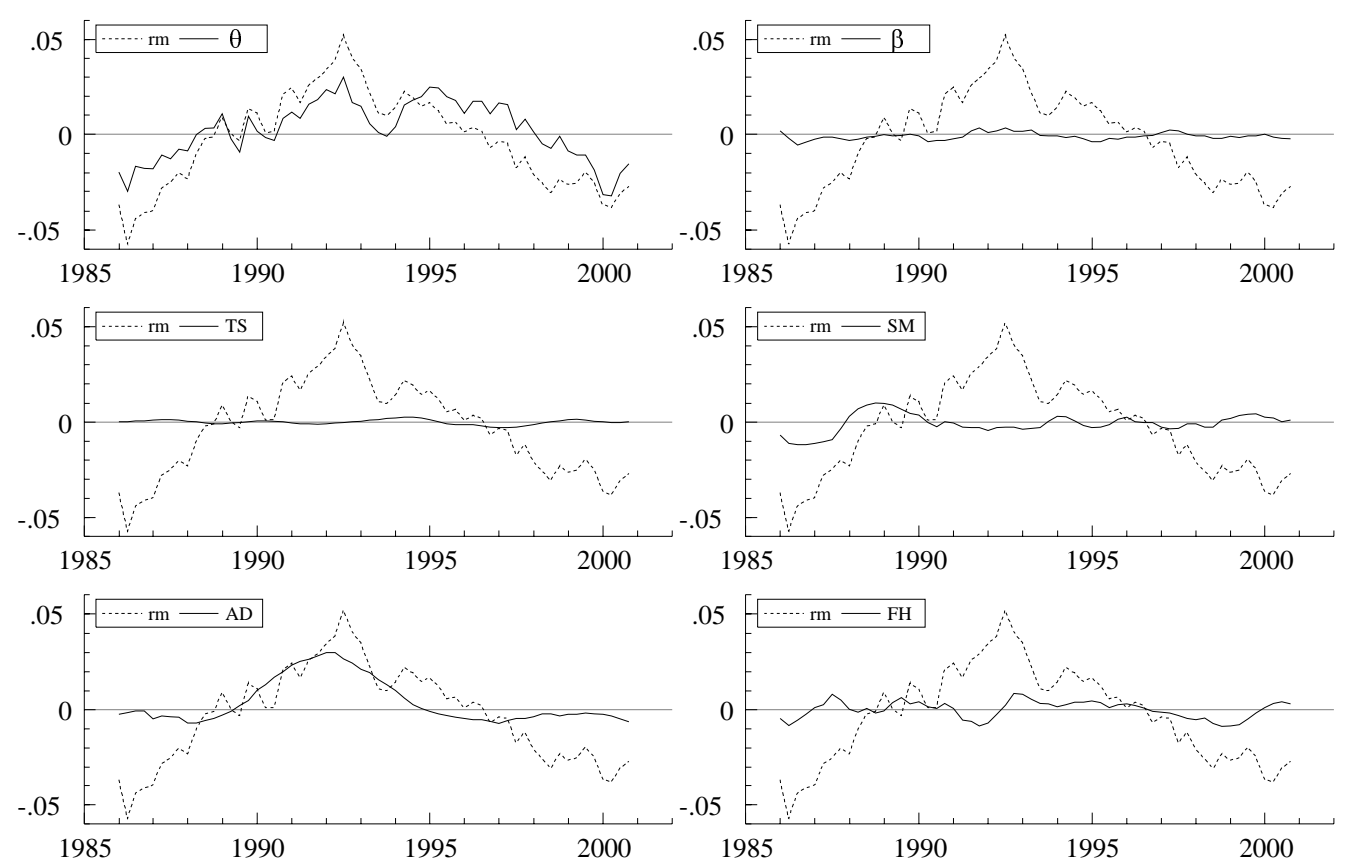

Figure 11: Historical decomposition of the real balances cycle $(\theta$ : real permanent shock; $\beta$ : nominal permanent shock, TS: term structure shock; SM: liquidity preference shock; AD: aggregate demand shock; FH: Fisher parity shock). 

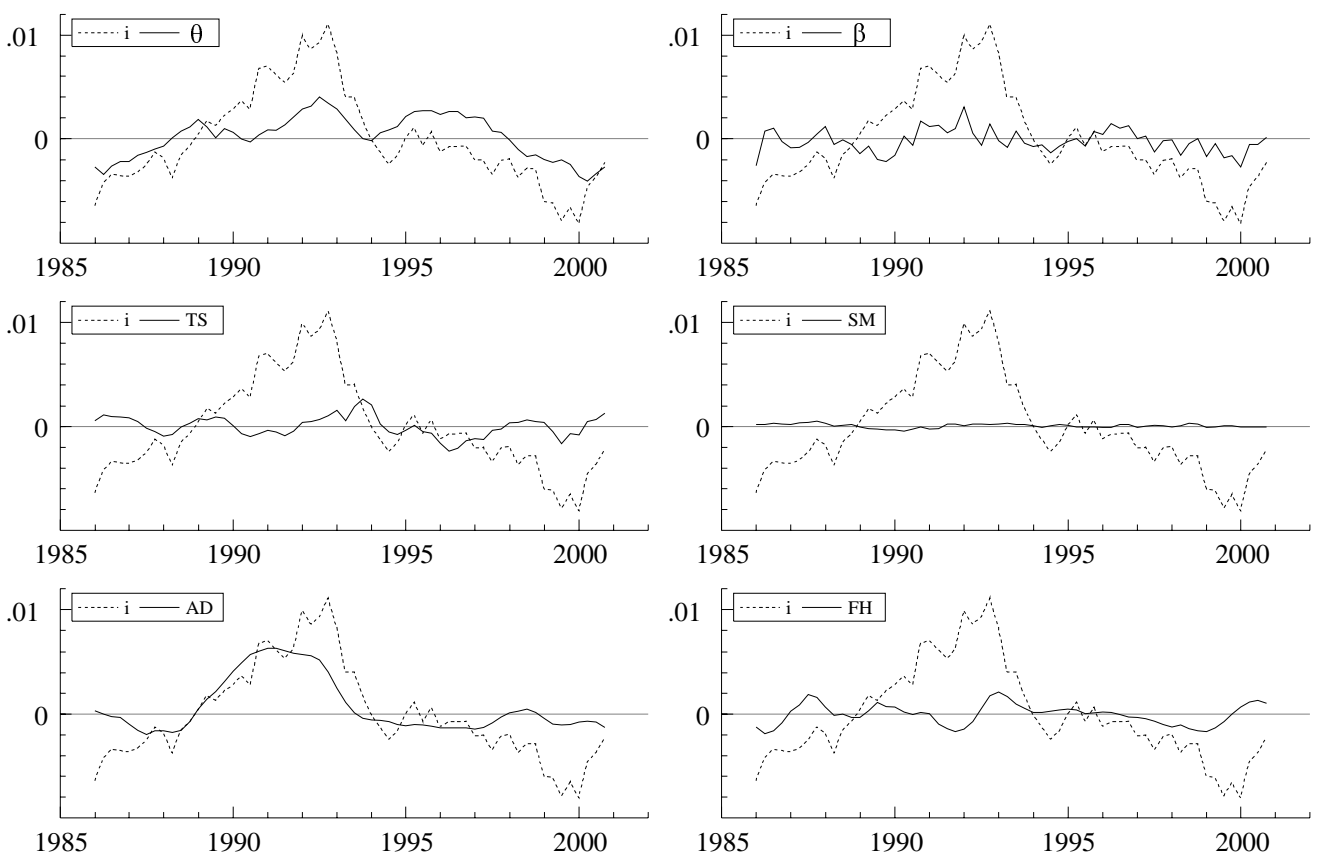

Figure 12: Historical decomposition of the short term rate cycle $(\theta$ : real permanent shock; $\beta$ : nominal permanent shock, TS: term structure shock; SM: liquidity preference shock; AD: aggregate demand shock; FH: Fisher parity shock). 

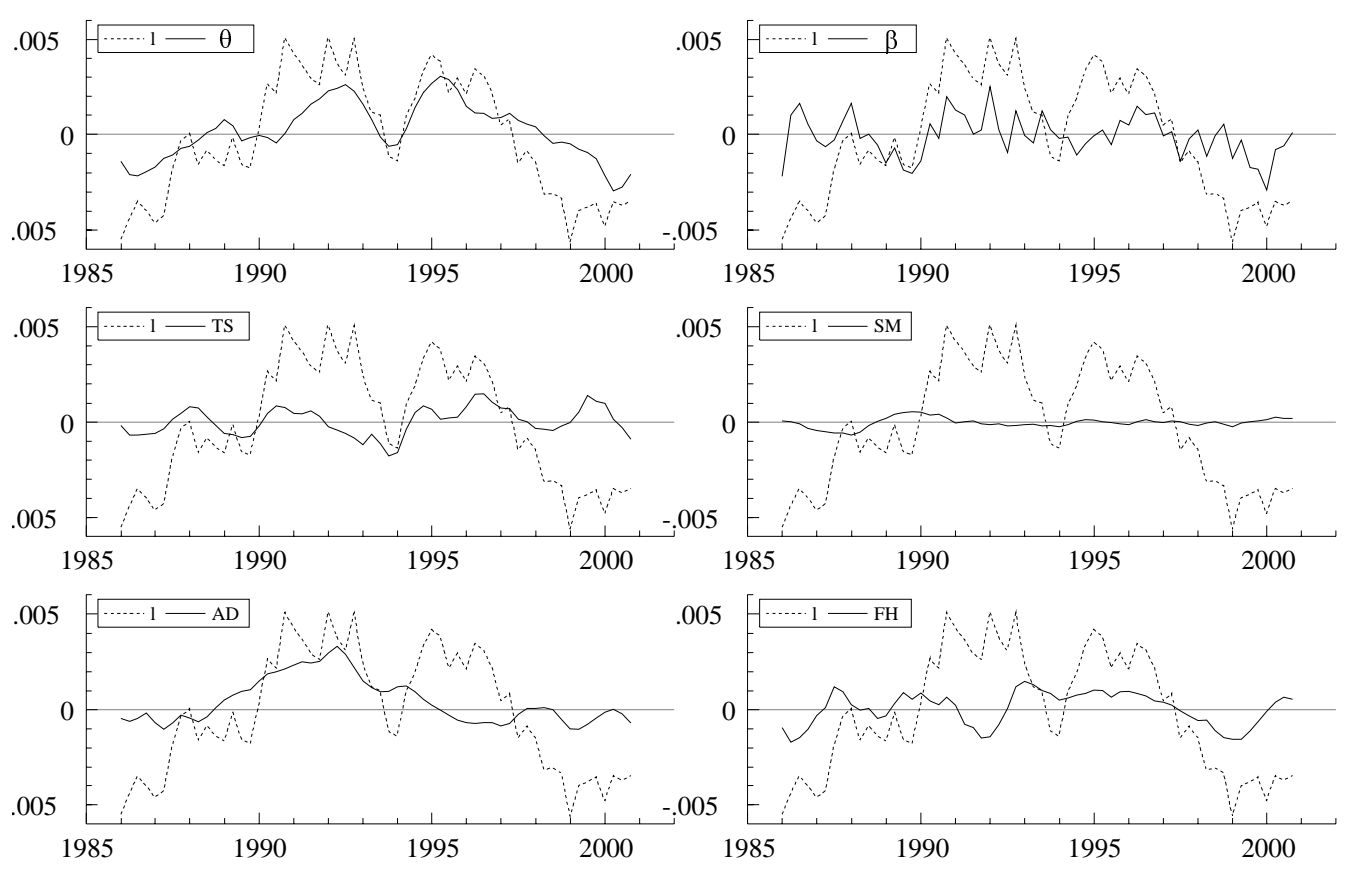

Figure 13: Historical decomposition of the long term rate cycle $\theta$ : real permanent shock; $\beta$ : nominal permanent shock, TS: term structure shock; SM: liquidity preference shock; AD: aggregate demand shock; FH: Fisher parity shock). 

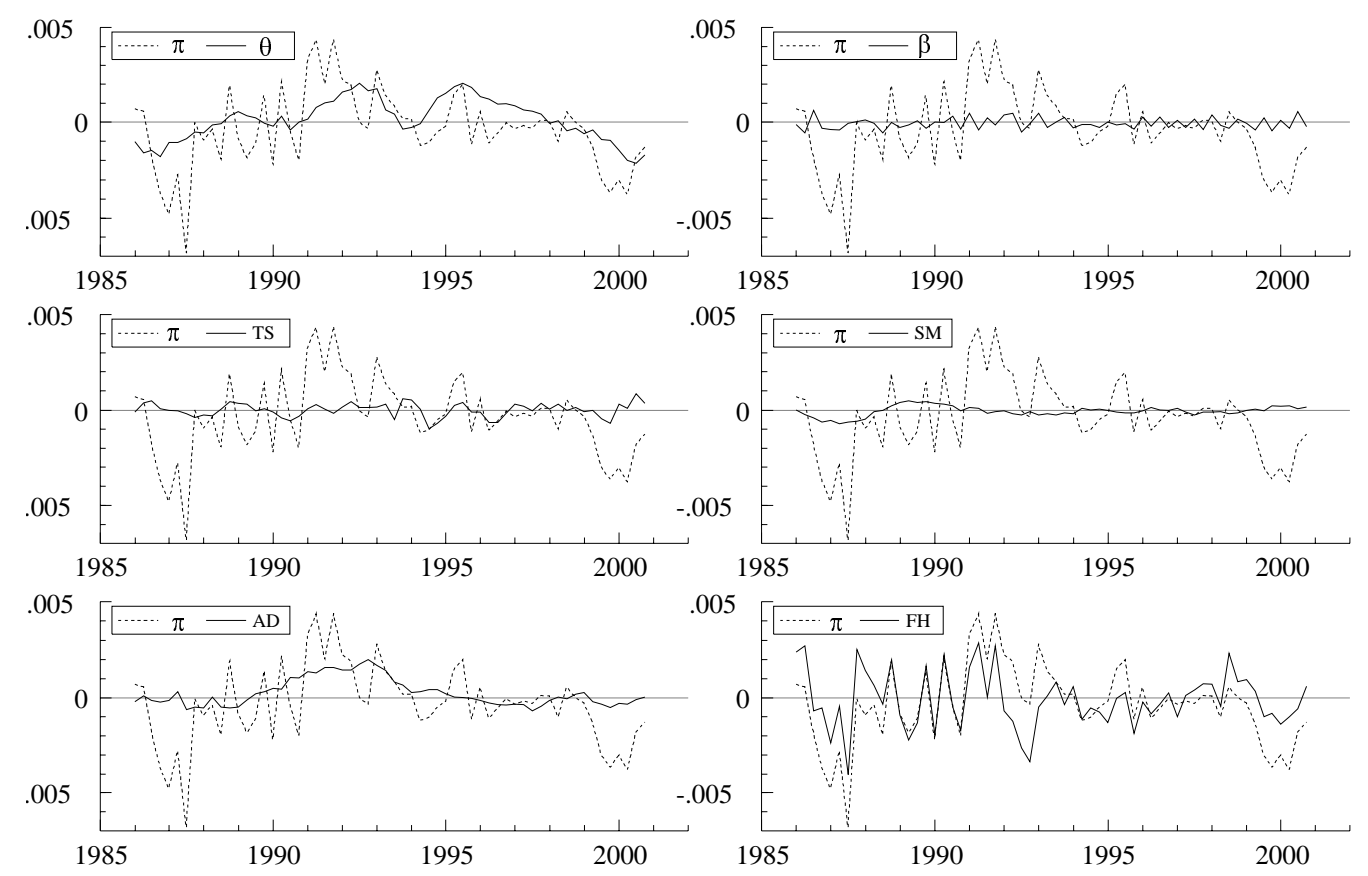

Figure 14: Historical decomposition of the inflation cycle $(\theta$ : real permanent shock; $\beta$ : nominal permanent shock, TS: term structure shock; SM: liquidity preference shock; AD: aggregate demand shock; FH: Fisher parity shock). 

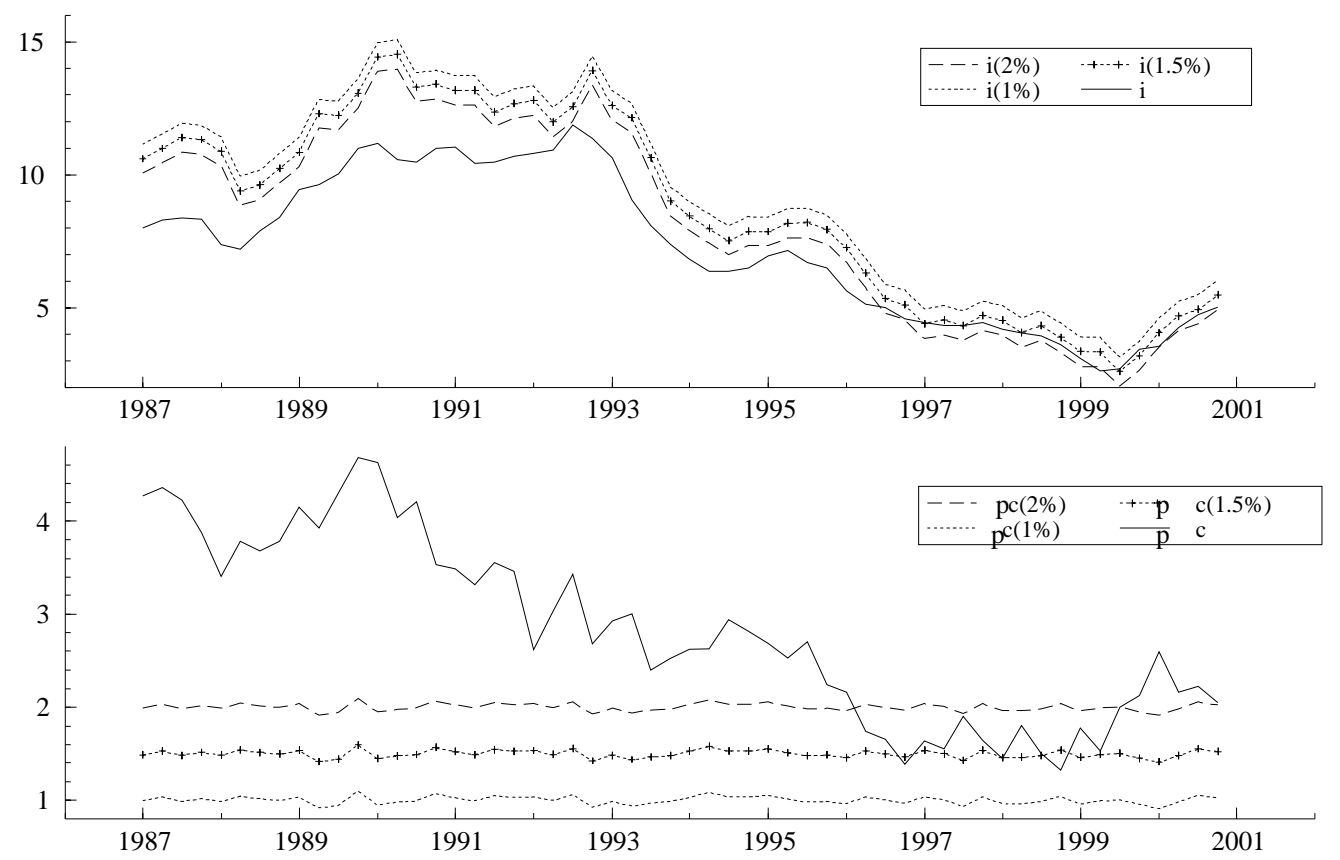

Figure 15: Unconditional simulation. Actual and simulated short-term interest rate, and core inflation rate. 


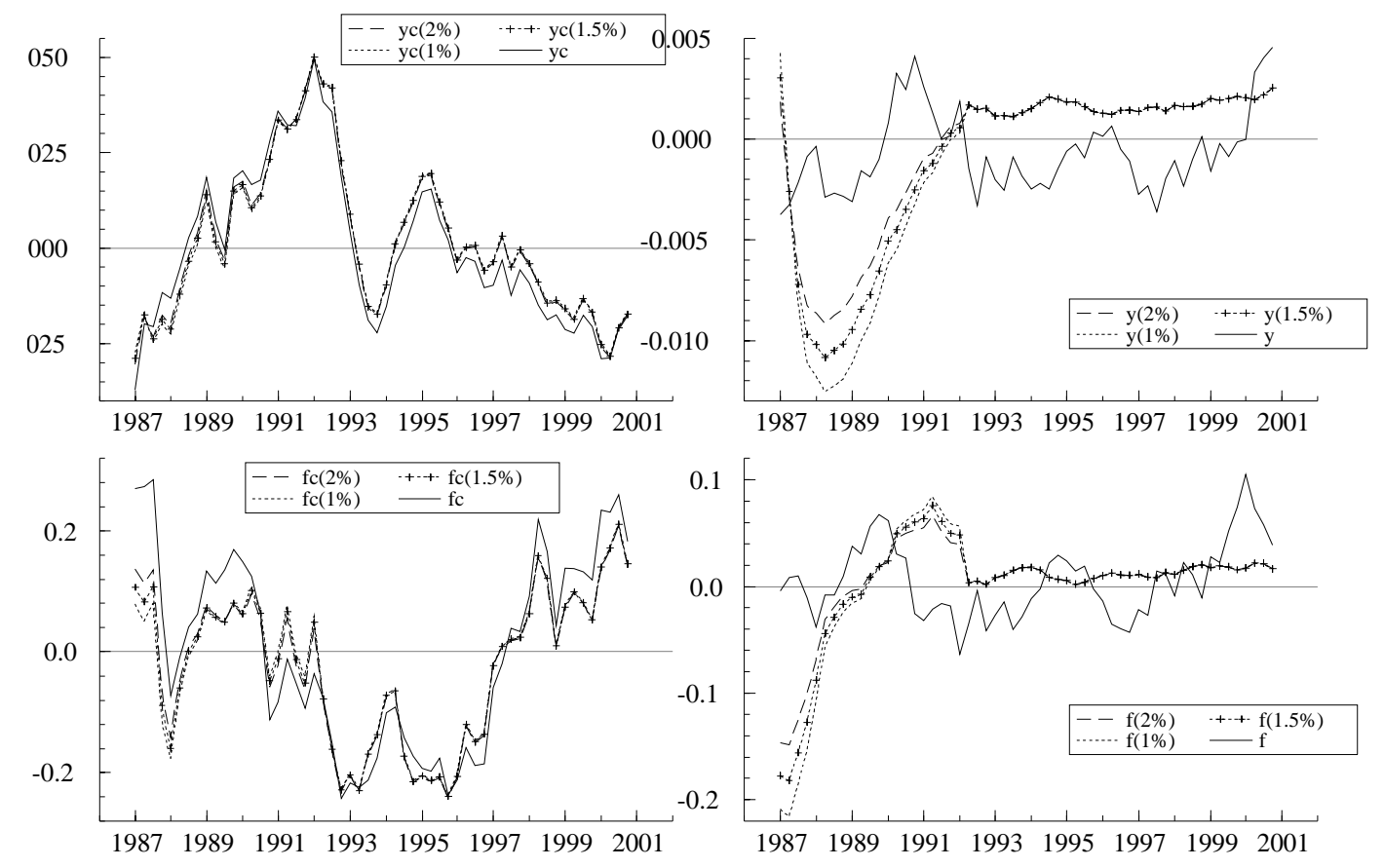

Figure 16: Conditional simulation. Left hand side panels: simulated and estimated cycles (yc, output; and fc, stock market). Right hand side panels: simulated and estimated contributions of nominal permanent innovations to the output (y) and stock market (f) cycles. 


\section{European Central Bank Working Paper Series}

I "A global hazard index for the world foreign exchange markets" by V. Brousseau and F. Scacciavillani, May 1999.

2 "What does the single monetary policy do? A SVAR benchmark for the European Central Bank" by C. Monticelli and O. Tristani, May 1999.

3 "Fiscal policy effectiveness and neutrality results in a non-Ricardian world" by C. Detken, May I999.

4 "From the ERM to the euro: new evidence on economic and policy convergence among EU countries" by I. Angeloni and L. Dedola, May 1999.

5 “Core inflation: a review of some conceptual issues" by M. Wynne, May 1999.

6 "The demand for M3 in the euro area" by G. Coenen and J.-L. Vega, September 1999.

7 “A cross-country comparison of market structures in European banking” by O. de Bandt and E. P. Davis, September 1999.

8 “Inflation zone targeting” by A. Orphanides and V. Wieland, October 1999.

9 "Asymptotic confidence bands for the estimated autocovariance and autocorrelation functions of vector autoregressive models" by G. Coenen, January 2000.

10 "On the effectiveness of sterilized foreign exchange intervention" by R. Fatum, February 2000.

II "Is the yield curve a useful information variable for the Eurosystem?" by J. M. Berk and P. van Bergeijk, February 2000.

12 “Indicator variables for optimal policy” by L. E. O. Svensson and M. Woodford, February 2000.

I3 “Monetary policy with uncertain parameters" by U. Söderström, February 2000.

14 "Assessing nominal income rules for monetary policy with model and data uncertainty" by G. D. Rudebusch, February 2000.

15 “The quest for prosperity without inflation” by A. Orphanides, March 2000.

16 "Estimating the implied distribution of the future short term interest rate using the Longstaff-Schwartz model” by P. Hördahl, March 2000.

17 "Alternative measures of the NAIRU in the euro area: estimates and assessment" by S. Fabiani and R. Mestre, March 2000.

18 "House prices and the macroeconomy in Europe: Results from a structural VAR analysis" by M. lacoviello, April 2000.

19 “The euro and international capital markets" by C. Detken and P. Hartmann, April 2000. 
20 "Convergence of fiscal policies in the euro area" by O. De Bandt and F. P. Mongelli, May 2000.

21 "Firm size and monetary policy transmission: evidence from German business survey data" by M. Ehrmann, May 2000.

22 "Regulating access to international large value payment systems" by C. Holthausen and T. Rønde, June 2000.

23 “Escaping Nash inflation” by In-Koo Cho and T. J. Sargent, June 2000.

24 “What horizon for price stability” by F. Smets, July 2000.

25 "Caution and conservatism in the making of monetary policy" by P. Schellekens, July 2000.

26 "Which kind of transparency? On the need for clarity in monetary policy-making" by B. Winkler, August 2000.

27 "This is what the US leading indicators lead" by M. Camacho and G. Perez-Quiros, August 2000.

28 "Learning, uncertainty and central bank activism in an economy with strategic interactions" by M. Ellison and N. Valla, August 2000.

29 "The sources of unemployment fluctuations: an empirical application to the Italian case" by S. Fabiani, A. Locarno, G. Oneto and P. Sestito, September 2000.

30 "A small estimated euro area model with rational expectations and nominal rigidities" by G. Coenen and V. Wieland, September 2000.

31 "The disappearing tax base: Is foreign direct investment eroding corporate income taxes?" by R. Gropp and K. Kostial, September 2000.

32 “Can indeterminacy explain the short-run non-neutrality of money?” by F. De Fiore, September 2000.

33 "The information content of M3 for future inflation" by C. Trecroci and J. L. Vega, October 2000.

34 "Capital market development, corporate governance and the credibility of exchange rate pegs" by O. Castrén and T. Takalo, October 2000.

35 "Systemic risk: A survey" by O. De Bandt and P. Hartmann, November 2000.

36 "Measuring core inflation in the euro area" by C. Morana, November 2000.

37 "Business fixed investment: Evidence of a financial accelerator in Europe" by P. Vermeulen, November 2000.

38 "The optimal inflation tax when taxes are costly to collect" by F. De Fiore, November 2000.

39 “A money demand system for euro area M3” by C. Brand and N. Cassola, November 2000.

40 "Financial structure and the interest rate channel of ECB monetary policy" by B. Mojon, November 2000. 
4I "Why adopt transparency? The publication of central bank forecasts” by P. M. Geraats, January 200 I.

42 “An area-wide model (AWM) for the euro area” by G. Fagan, J. Henry and R. Mestre, January 200I.

43 "Sources of economic renewal: from the traditional firm to the knowledge firm" by D. R. Palenzuela, February 200I.

44 "The supply and demand for eurosystem deposits - The first 18 months" by U. Bindseil and F. Seitz, February 200I.

45 "Testing the Rank of the Hankel matrix: a statistical approach" by G. Camba-Mendez and G. Kapetanios, February 200I.

46 "A two-factor model of the German term structure of interest rates” by N. Cassola and J. B. Luís, February 2001 .

47 "Deposit insurance and moral hazard: does the counterfactual matter?" by R. Gropp and J. Vesala, February 2001 .

48 "Financial market integration in Europe: on the effects of EMU on stock markets" by M. Fratzscher, March 2001.

49 "Business cycle and monetary policy analysis in a structural sticky-price model of the euro area" by M. Casares, March $200 \mathrm{I}$.

50 "Employment and productivity growth in service and manufacturing sectors in France, Germany and the US” by T. von Wachter, March 200I.

5I "The functional form of the demand for euro area MI" by L. Stracca, March $200 \mathrm{I}$.

52 "Are the effects of monetary policy in the euro area greater in recessions than in booms?" by G. Peersman and F. Smets, March 2001 .

53 "An evaluation of some measures of core inflation for the euro area" by J.-L. Vega and M. A. Wynne, April 2001.

54 “Assessment criteria for output gap estimates” by G. Camba-Méndez and D. R. Palenzuela, April 200I.

55 "Modelling the demand for loans to the private sector in the euro area" by A. Calza, G. Gartner and J. Sousa, April 200I.

56 "Stabilization policy in a two country model and the role of financial frictions" by E. Faia, April 200I.

57 “Model-based indicators of labour market rigidity” by S. Fabiani and D. Rodriguez-Palenzuela, April 200I.

58 "Business cycle asymmetries in stock returns: evidence from higher order moments and conditional densities” by G. Perez-Quiros and A. Timmermann, April 200I.

59 "Uncertain potential output: implications for monetary policy” by M. Ehrmann and F. Smets, April 200 I. 
60 "A multi-country trend indicator for euro area inflation: computation and properties" by E. Angelini, J. Henry and R. Mestre, April 2001.

61 "Diffusion index-based inflation forecasts for the euro area" by E. Angelini, J. Henry and R. Mestre, April 2001.

62 "Spectral based methods to identify common trends and common cycles" by G. C. Mendez and G. Kapetanios, April 200I.

63 "Does money lead inflation in the euro area?" by S. N. Altimari, May 200 I.

64 "Exchange rate volatility and euro area imports" by R. Anderton and F. Skudelny, May 200I.

65 "A system approach for measuring the euro area NAIRU” by S. Fabiani and R. Mestre, May $200 \mathrm{I}$.

66 "Can short-term foreign exchange volatility be predicted by the Global Hazard Index?" by V. Brousseau and F. Scacciavillani, June $200 \mathrm{I}$.

67 "The daily market for funds in Europe: Has something changed with the EMU?" by G. P. Quiros and H. R. Mendizabal, June 200 I.

68 "The performance of forecast-based monetary policy rules under model uncertainty" by A. Levin, V. Wieland and J. C.Williams, July $200 \mathrm{I}$.

69 "The ECB monetary policy strategy and the money market” by V. Gaspar, G. Perez-Quiros and J. Sicilia, July $200 \mathrm{I}$.

70 "Central Bank forecasts of liquidity factors: Quality, publication and the control of the overnight rate" by U. Bindseil, July 200I.

7I “Asset market linkages in crisis periods” by P. Hartmann, S. Straetmans and C. G. de Vries, July 200 I.

72 "Bank concentration and retail interest rates" by S. Corvoisier and R. Gropp, July 200 I.

73 "Interbank lending and monetary policy transmission - evidence for Germany" by M. Ehrmann and A. Worms, July 2001 .

74 “Interbank market integration under asymmetric information" by X. Freixas and C. Holthausen, August 2001.

75 "Value at risk models in finance" by S. Manganelli and R. F. Engle, August 200I.

76 "Rating agency actions and the pricing of debt and equity of European banks: What can we infer about private sector monitoring of bank soundness?” by R. Gropp and A. J. Richards, August 200 I.

77 “Cyclically adjusted budget balances: An alternative approach" by C. Bouthevillain, P. Cour-Thimann, G. van den Dool, P. Hernández de Cos, G. Langenus, M. Mohr, S. Momigliano and M. Tujula, September $200 \mathrm{I}$. 
78 "Investment and monetary policy in the euro area" by B. Mojon, F. Smets and P. Vermeulen, September 200I.

79 "Does liquidity matter? Properties of a synthetic divisia monetary aggregate in the euro area" by L. Stracca, October 200 I.

80 "The microstructure of the euro money market" by P. Hartmann, M. Manna and A. Manzanares, October 2001.

81 "What can changes in structural factors tell us about unemployment in Europe?" by J. Morgan and A. Mourougane, October $200 \mathrm{I}$.

82 "Economic forecasting: some lessons from recent research" by D. Hendry and M. Clements, October 200I.

83 "Chi-squared tests of interval and density forecasts, and the Bank of England's fan charts" by K. F. Wallis, November 200I.

84 "Data uncertainty and the role of money as an information variable for monetary policy" by G. Coenen, A. Levin and V. Wieland, November 2001.

85 "Determinants of the euro real effective exchange rate: a BEER/PEER approach" by F. Maeso-Fernandez, C. Osbat and B. Schnatz, November 2001.

86 "Rational expectations and near rational alternatives: how best to form expecations" by M. Beeby, S. G. Hall and S. B. Henry, November 200 I.

87 "Credit rationing, output gap and business cycles" by F. Boissay, November 2001.

88 "Why is it so difficult to beat the random walk forecast of exchange rates?" by L. Kilian and M. P. Taylor, November 2001.

89 "Monetary policy and fears of financial instability" by V. Brousseau and C. Detken, November 2001.

90 "Public pensions and growth" by S. Lambrecht, P. Michel and J.-P. Vidal, November 200I.

91 "The monetary transmission mechanism in the euro area: more evidence from VAR analysis" by G. Peersman and F. Smets, December 2001.

92 "A VAR description of the effects of monetary policy in the individual countries of the euro area" by B. Mojon and G. Peersman, December 2001.

93 "The monetary transmission mechanism at the euro-area level: issues and results using structural macroeconomic models" by P. McAdam and J. Morgan, December $200 \mathrm{I}$.

94 "Monetary policy transmission in the euro area: what do aggregate and national structural models tell us?" by P. van Els, A. Locarno, J. Morgan and J.-P. Villetelle, December $200 I$.

95 "Some stylised facts on the euro area business cycle" by A.-M. Agresti and B. Mojon, December 200I.

96 "The reaction of bank lending to monetary policy measures in Germany" by A. Worms, December 2001. 
97 “Asymmetries in bank lending behaviour. Austria during the 1990s” by S. Kaufmann, December 200 I.

98 "The credit channel in the Netherlands: evidence from bank balance sheets" by L. De Haan, December 200I.

99 "Is there a bank lending channel of monetary policy in Spain?" by I. Hernando and J. Martínez-Pagés, December 200I.

100 "Transmission of monetary policy shocks in Finland: evidence from bank level data on loans" by J. Topi and J. Vilmunen, December 200I.

I0I “Monetary policy and bank lending in France: are there asymmetries?” by C. Loupias, F. Savignac and P. Sevestre, December 2001 .

102 "The bank lending channel of monetary policy: identification and estimation using Portuguese micro bank data" by L. Farinha and C. Robalo Marques, December $200 \mathrm{I}$.

103 "Bank-specific characteristics and monetary policy transmission: the case of Italy" by L. Gambacorta, December 2001.

104 "Is there a bank lending channel of monetary policy in Greece? Evidence from bank level data" by S. N. Brissimis, N. C. Kamberoglou and G. T. Simigiannis, December 200 I.

105 "Financial systems and the role of banks in monetary policy transmission in the euro area" by M. Ehrmann, L. Gambacorta, J. Martínez-Pagés, P. Sevestre and A. Worms, December 200 I.

106 "Investment, the cost of capital, and monetary policy in the nineties in France: a panel data investigation" by J.-B. Chatelain and A. Tiomo, December 2001.

107 "The interest rate and credit channel in Belgium: an investigation with micro-level firm data" by P. Butzen, C. Fuss and P. Vermeulen, December 2001.

108 "Credit channel and investment behaviour in Austria: a micro-econometric approach" by M. Valderrama, December 2001.

109 "Monetary transmission in Germany: new perspectives on financial constraints and investment spending" by U. von Kalckreuth, December 2001.

I 10 "Does monetary policy have asymmetric effects? A look at the investment decisions of Italian firms" by E. Gaiotti and A. Generale, December 200I.

III "Monetary transmission: empirical evidence from Luxembourg firm level data" by P. Lünnemann and T. Mathä, December 2001.

I 12 "Firm investment and monetary transmission in the euro area" by J.-B. Chatelain, A. Generale, I. Hernando, U. von Kalckreuth and P. Vermeulen, December $200 \mathrm{I}$.

II4 “Monetary transmission in the euro area: where do we stand?" by I. Angeloni, A. Kashyap, B. Mojon, D. Terlizzese, January 2002. 
II5 “Monetary policy rules, macroeconomic stability and inflation: a view from the trenches" by A. Orphanides, December $200 \mathrm{I}$.

116 "Rent indices for housing in West Germany 1985 to 1998” by J. Hoffmann and C. Kurz., January 2002.

117 "Hedonic house prices without characteristics: the case of new multiunit housing" by O. Bover and P. Velilla, January 2002.

I 18 "Durable goods, price indexes and quality change: an application to automobile prices in Italy, 19881998” by G. M. Tomat, January 2002.

II9 "Monetary Policy and the Stock Market in the Euro Area" by N. Cassola and C. Morana, January 2002. 